\title{
PRODUCING 3D RICCI FLOWS WITH NON-NEGATIVE RICCI CURVATURE VIA SINGULAR RICCI FLOWS
}

\author{
YI LAI
}

\begin{abstract}
We extend the concept of singular Ricci flow by Kleiner and Lott from $3 \mathrm{~d}$ compact manifolds to $3 \mathrm{~d}$ complete manifolds with possibly unbounded curvature. As an application of the generalized singular Ricci flow, we show that for any $3 \mathrm{~d}$ complete Riemannian manifold with non-negative Ricci curvature, there exists a smooth Ricci flow starting from it. This partially confirms a conjecture by Topping.
\end{abstract}

\section{INTRODUCTION AND MAIN RESULTS}

The Ricci flow was introduced by Hamilton in [Ham82]. Since its singularities can occur along proper subsets of the manifold, in order to continue the flow, Hamilton introduced Ricci flow with surgery in [Ham86]. Based on the earlier work of Hamilton, Perelman constructed Ricci flow with surgery on any compact 3 dimensional Riemannian manifold, and hence proved the Geometrization and Poincaré Conjectures in [Per02] Per03a] Per03b]. After this, the Ricci flow with surgery was also constructed for complete non-compact manifolds with bounded geometry by Bessière, Besson, and Maillot in [BBM11].

Perelman's Ricci flow with surgery is a sequence of ordinary compact Ricci flows such that the final time-slice of each flow is isometric, modulo surgery, to the initial time-slice of the next one. The surgery process is regulated by several parameters, one of them being the surgery scale $\delta>0$. Perelman showed that $\delta>0$ can be chosen arbitrarily small, and as such he conjectured that the Ricci flow with surgery should converge to a canonical Ricci flow through singularities.

Recently, such a canonical flow, named the singular Ricci flow, was constructed by Kleiner and Lott in [KL17, and shown to be unique by Bamler and Kleiner in BK17b. The singular Ricci flow is a 0-complete Ricci flow spacetime starting from a compact manifold, which satisfies the Hamilton-Ivey pinching, and the canonical neighborhood assumption at scales less than a time-dependent constant.

In this paper, we introduce a new weak solution of Ricci flow that we call a generalized singular Ricci flow, which allows the initial manifold to be a complete manifold with possibly unbounded curvature. We have the following existence theorem.

Theorem 1.1. For any $3 d$ complete Riemannian manifold $(M, g)$, there is a generalized singular Ricci flow starting from $(M, g)$. 
The generalized singular Ricci flow has many properties similar to those of a singular Ricci flow. In particular, it satisfies the canonical neighborhood assumption in a distance-dependent way. Its precise definition of a generalized singular Ricci flow will be given in Definition 7.2.

The existence is obtained from a compactness result for singular Ricci flows, which states that a sequence of singular Ricci flows converges to a generalized singular Ricci flow starting from a complete manifold $(M, g)$, if the sequence of their initial manifolds converges to $(M, g)$ :

Theorem 1.2. Let $\mathcal{M}_{i}$ be a sequence of singular Ricci flows starting from compact manifolds $M_{i}, x_{i} \in M_{i}$. Suppose $\left(M_{i}, x_{i}\right)$ converges smoothly to a $3 d$ complete manifold $\left(M, x_{0}\right)$ as $i \rightarrow \infty$. Then by passing to a subsequence, $\left(\mathcal{M}_{i}, x_{i}\right)$ converges smoothly to a generalized singular Ricci flow starting from $M$.

Theorem 1.2 can be compared to the convergence of a sequence of singular Ricci flows, when the sequence of their initial time-slices converges to a compact manifold, see [KL17, Prop 5.39]. In that result, the initial time-slices have uniformly bounded curvature and injectivity radius. So the local geometry in each singular Ricci flow is uniformly controlled by its scalar curvature, which guarantees their convergence to a singular Ricci flow. However, in our case, the initial time-slices may not have uniformly bounded geometry. Instead, we will show that the scalar curvature controls the local geometry in a uniform distance-dependent way, which ensures the convergence in Theorem 1.2.

Before stating our next main result, we recall some results of the existence theory of Ricci flow with non-compact initial conditions. Much less is known about it compared to the compact case. In [Shi87], Shi showed that if $(M, g)$ is an n-dimensional complete Riemannian manifold with bounded curvature, then there exists a complete Ricci flow with bounded curvature for a short time. Since then, many efforts have been made to relax the bounded-curvature assumption, in order to obtain a Ricci flow starting from a complete non-compact manifold.

In CRW15, Cebazas-Rivas and Wilking proved that a smooth complete Ricci flow exists on a complete n-dimensional manifold with non-negative complex sectional curvature, which in dimension 3 is the same as non-negative sectional curvature. Recently, Simon and Topping [ST17] showed that a complete Ricci flow exists on a complete 3d Riemannian manifold, if its Ricci curvature has a negative lower bound and the volume is globally non-collapsed (i.e. there is a uniform positive lower bound on the volume of every unit ball). In [BCRW19], Bamler, Cebazas-Rivas and Wilking proved that the same thing holds in dimension $n$, assuming a certain curvature is bounded below, and the volume is non-collapsed. In [Lai19, by a combination of methods in [ST17] and [BCRW19], the author generalized both works.

The volume non-collapsing assumption is necessary in [ST17][BCRW19][Lai19], where the curvature is allowed to be negative somewhere. For example, see [Top19, Example 2.4], for any arbitrarily small $\epsilon>0$ we can construct a complete 3-manifold 
with Ric $\geq-\epsilon$, by connecting countably many three-spheres by necks that become longer and thinner. So the necks would pinch in times converging to zero, and hence a Ricci flow cannot exist for any short time.

This leads to an open question: whether a smooth complete Ricci flow exists for a 3 dimensional complete manifold with non-negative Ricci curvature, see e.g. Top19, Conjecture 7.1]. Our next main result gives a partial affirmative answer to this question:

Theorem 1.3. Let $(M, g)$ be a $3 d$ complete Riemannian manifold with Ric $\geq 0$. There exist $T>0$ and a smooth Ricci flow $(M, g(t))$ on $[0, T)$, with $g(0)=g$ and $\operatorname{Ric}(g(t)) \geq 0$. Moreover, if $T<\infty$, then $\lim \sup _{t \nearrow_{T}}|\operatorname{Rm}|(x, t)=\infty$ for all $x \in M$.

We remark that the completeness of this flow is not guaranteed in this paper. Instead, we show that it can be embedded in a smooth Ricci flow spacetime with complete time-slices. Also, it is possible for the maximal existence time to be finite, such as the standard solution and the cylindrical solutions.

A common strategy to produce a smooth Ricci flow with a complete non-compact initial condition is by a limiting argument: first construct a sequence of local Ricci flows starting from larger and larger balls in $M$, and then try to get a uniform lower bound on the existence times, as well as an upper bound on the curvature norms. Then by Hamilton's compactness theorem for Ricci flow, we obtain a smooth limit Ricci flow starting from $M$. This argument typically works when there is a non-collapsing assumption [ST17, BCRW19, Lai19], or the curvature condition is relatively strong CRW15.

However, it seems hard to apply the limiting argument to prove Theorem 1.3, for Ric $\geq 0$ is a relatively weak curvature assumption, and there is no uniform lower bound on the volume of all unit balls on certain manifolds, as shown by examples in CK88. In this paper, we produce a smooth Ricci flow by showing that a generalized singular Ricci flow starting from a complete manifold with Ric $\geq 0$ is actually smooth. The existence of the generalized singular Ricci flow is guaranteed by Theorem 1.1.

The paper is organized as follows. In Section 2, we review some basic concepts in Perelman's Ricci flow with surgery and the singular Ricci flow. Section 3 is for some technical lemmas. In Section 4, we generalize Perelman's no local collapsing and canonical neighborhood theorem to singular Ricci flows. It provides a distancedependent lower bound on the non-collapsing scale and canonical neighborhood scale, assuming the geometry is bounded in a parabolic neighborhood of the base point.

In Section 5, we define a heat kernel $H$ for a singular Ricci flow $\mathcal{M}$. For any point $\left(x_{0}, t_{0}\right) \in \mathcal{M}, H\left(x_{0}, t_{0} ; \cdot, \cdot\right)$ is a positive solution to the conjugate heat equation on $\mathcal{M}$, which is a $\delta$-function around $\left(x_{0}, t_{0}\right)$. Moreover, we show that the heat kernel decays polynomially fast to zero as the curvature blows up. This implies that the overall amount of heat is a constant, i.e. the integral of $H\left(x_{0}, t_{0} ; \cdot, t\right)$ at all times $t$ prior to $t_{0}$ is equal to one. Note that for the ordinary heat kernel of a compact smooth Ricci flow, the constancy of the integral is easily shown by a computation using integration by 
part. Moreover, the polynomial decay near the high curvature region also implies that Perelman's Harnack inequality holds for singular Ricci flow. With these properties of the heat kernel, we are able to generalize Perelman's pseudolocality theorem to singular Ricci flow in Section 6 .

In Section 7, we define the generalized singular Ricci flow, and prove Theorem 1.1 and 1.2. The proofs depend on a compactness theorem, which states that assuming there is a uniform distance-dependent canonical neighborhood assumption in a sequence of pointed singular Ricci flows, then a subsequence converges smoothly to a semi-generalized singular Ricci flow, which satisfies most properties of the generalized singular Ricci flow. The compactness theorem can be proved by first taking a GromovHausdorff limit, and showing that the convergence is smooth on the subset of points which are limits of points with bounded curvature. This induces a semi-generalized singular Ricci flow. To prove Theorem 1.2, by applying the compactness theorem, we get a semi-generalized singular Ricci flow in which the base point $x_{0}$ survives until its curvature goes unbounded. Then a generalized singular Ricci flow is obtained by varying the base points and gluing up all the corresponding semi-generalized singular Ricci flows.

In Section 8 we prove Theorem 1.3. First, by a maximum principle argument, we show in Lemma 8.2 that the generalized singular Ricci flow $\mathcal{M}$ preserves the nonnegativity of Ricci curvature. Suppose the curvature blows up in a ball of finite radius. Then by the canonical neighborhood assumption, we can show that the curvature blow-up is due to the asymptotic formation of a cone-like point. Doing a further rescaling at this cone-like point, we obtain a Ricci flow solution whose final time-slice is a part of a non-flat metric cone, which is impossible. So $\mathcal{M}$ is in fact a non-singular Ricci flow spacetime with complete time-slices. Restricting the spacetime on $M$, we obtain a smooth Ricci flow.

I thank my advisor Richard Bamler for helpful discussions and many comments. I also thank John Lott and Guoqing Wu for comments, and Paula Burkhardt and Angxiu Ni for correcting my English.

\section{Preliminary}

In this section, we collect some notions and concepts of Perelman's Ricci flow with surgery [KL08] and singular Ricci flow [KL17], [BK17a] that will be frequently used later.

\subsection{Ricci flow spacetime.}

Definition 2.1 (Ricci flow spacetime). A Ricci flow spacetime is a tuple $\left(\mathcal{M}, \mathfrak{t}, \partial_{\mathfrak{t}}, g\right)$ (sometimes abbreviate as $\mathcal{M}$ or $(\mathcal{M}, g(t)))$ with the following properties:

(1) $\mathcal{M}$ is a smooth 4-manifold with (smooth) boundary $\partial \mathcal{M}$.

(2) $\mathfrak{t}: \mathcal{M} \rightarrow[0, T)$, where $T$ can be infinity, is a smooth function without critical points. For any $t \geq 0$ we denote by $\mathcal{M}_{t}:=\mathfrak{t}^{-1}(t) \subset \mathcal{M}$ the time-t-slice of $\mathcal{M}$. 
(3) $\partial \mathcal{M}=\mathcal{M}_{0}$, i.e. the boundary of $\mathcal{M}$ is equal to the initial time-slice.

(4) $\partial_{\mathfrak{t}}$ is a smooth vector field (the time vector field), which satisfies $\partial_{\mathfrak{t}} \mathfrak{t} \equiv 1$.

(5) $g$ is a smooth inner product on the spacial subbundle $\operatorname{ker}(d \mathfrak{t}) \subset T \mathcal{M}$. For any $t \geq 0$ we denote by $g(t)$ the restriction of $g$ to the time-t-slice $\mathcal{M}_{t}$, which is a Riemannian metric.

(6) $g$ satisfies the Ricci flow equation: $\mathcal{L}_{\partial_{t}} g=-2 \operatorname{Ric}(g(t))$.

We call the Riemannian metric $G:=d t^{2}+g$ the spacetime metric.

Definition 2.2 (Points in a Ricci flow spacetime). Let $\left(\mathcal{M}, \mathfrak{t}, \partial_{\mathfrak{t}}, g\right)$ be a Ricci flow spacetime and $x \in \mathcal{M}$ be a point. Set $t:=\mathfrak{t}(x)$. We sometimes write $x$ as $(x, t)$ to indicate its time, when there is no ambiguity. Consider the maximal trajectory $\gamma_{x}: I \rightarrow \mathcal{M}, I \subset[0, \infty)$ of the time-vector field $\partial_{\mathfrak{t}}$ such that $\gamma_{x}(t)=x$. Note that $\mathfrak{t}\left(\gamma_{x}\left(t^{\prime}\right)\right)=t^{\prime}$ for all $t^{\prime} \in I$. For any $t^{\prime} \in I$ we say that $x$ survives until time $t^{\prime}$ and we write

$$
x\left(t^{\prime}\right):=\gamma_{x}\left(t^{\prime}\right)
$$

Similarly, for a subset $X \subset \mathcal{M}_{t}$, we say that $X$ survives until time $t^{\prime}$ if this is true for every $x \in X$, and we write $X\left(t^{\prime}\right)=\left\{x\left(t^{\prime}\right): x \in X\right\}$.

Definition 2.3 (Distance and metric balls). Let $\left(\mathcal{M}, \mathfrak{t}, \partial_{\mathfrak{t}}, g\right)$ be a Ricci flow spacetime. For any two points $x, y \in \mathcal{M}_{t}$ we denote by $d_{t}(x, y)$, or simply $d(x, y)$ the distance between $x, y$ within $\left(\mathcal{M}_{t}, g(t)\right)$.

For any $x \in \mathcal{M}_{t}$ and $r \geq 0$ we denote by $B_{t}(x, r) \subset \mathcal{M}_{t}$ the $r$-ball around $x$ with respect to the Riemannian metric $g(t)$.

Definition 2.4 (Parabolic neighborhood). Let $\left(\mathcal{M}, \mathfrak{t}, \partial_{\mathfrak{t}}, g\right)$ be a Ricci flow spacetime. For any $y \in \mathcal{M}$ let $I_{y} \subset[0, \infty)$ be the set of all times until which $y$ survives. Let $x \in \mathcal{M}$ and $a \geq 0, b \in \mathbb{R}$. Set $t=\mathfrak{t}(x)$. Then we define the parabolic neighborhood $P(x, a, b) \subset \mathcal{M}$ to be:

$$
P(x, a, b):=\bigcup_{y \in B_{t}(x, a)} \bigcup_{t^{\prime} \in[t, t+b] \cap I_{y}} y\left(t^{\prime}\right) .
$$

If $b<0$, we replace $[t, t+b]$ by $[t+b, t]$. We call $P(x, a, b)$ unscathed if $B(x, a)$ is relatively compact in $\mathcal{M}_{t}$ and if $B(x, a)$ survives until $t+b$.

Definition 2.5 (Admissible curve and accessibility). Let $\mathcal{M}$ be a Ricci flow spacetime, we say $\gamma:[c, d] \rightarrow \mathcal{M}$ is an admissible curve if $\gamma(t) \in \mathcal{M}_{t}$ for all $t \in[c, d]$. We say a point $x \in \mathcal{M}$ with $\mathfrak{t}(x)<\mathfrak{t}\left(x_{0}\right)$ is accessible from $x_{0}$ if there is an admissible curve running from $(x, t)$ to $\left(x_{0}, t_{0}\right)$.

Let $x_{0} \in \mathcal{M}_{t}, t>0$. We denote by $\mathcal{M}\left(x_{0}\right)$ the subset consisting of all points in $\mathcal{M}$ that are accessible to $x_{0}$.

Definition 2.6 (Hamilton-Ivey pinching). Let $M$ be a 3 dimensional Riemannian manifold and $\varphi>0$. We say that the curvature at $x \in M$ is $\varphi$-positive if there is an 
$X>0$ with $\operatorname{Rm}(x) \geq-X$ such that

$$
R(x) \geq-\frac{3}{\varphi^{-1}} \quad \text { and } \quad R(x) \geq X\left(\log X+\log \left(\varphi^{-1}\right)-3\right) .
$$

Let $(M, g(t)), t \in[0, T]$ be a 3 dimensional compact Ricci flow and $\varphi \in \mathbb{R}_{+} \cup \infty$. We say that the curvature at $(x, t) \in M \times[0, T]$ is $\varphi$-positive if there is an $X>0$ with $\operatorname{Rm}(x, t) \geq-X$ such that

$$
R(x, t) \geq-\frac{3}{\varphi^{-1}+t} \quad \text { and } \quad R(x, t) \geq X\left(\log X+\log \left(\varphi^{-1}+t\right)-3\right) .
$$

The Hamilton-Ivey pinching theorem [KL08, Appendix B] says that if the curvature is $\varphi$-positive at time 0 , then the curvature is $\varphi$-positive at all positive times. Moreover, the same conclusion also holds for singular Ricci flow [KL17, Theorem 1.3].

Definition 2.7 ( $\kappa$-non-collapsed). Let $(M, g)$ be a 3 dimensional Riemannian manifold, $x \in M$ and $\kappa, r_{0}>0$. We say $M$ is $\kappa$-non-collapsed at $x$ at scales less than $r_{0}$, if $r^{-3} \operatorname{vol}\left(B_{g}(x, r)\right) \geq \kappa>0$, for all $0<r \leq r_{0}$ such that $|\mathrm{Rm}| \leq r^{-2}$ holds on $B_{g}(x, r)$.

Definition 2.8 (Normalized manifold). Let $(M, g)$ be a 3-dimensional compact orientable connected Riemannian manifold that

(1) is not a higher spherical space form,

(2) has scalar curvature $R<1$ everywhere,

(3) is 1-non-collapsed at scales less than 1 and

(4) satisfies the 1-positive curvature condition at time 0.

Then we say $(M, g)$ has normalized geometry. For a Ricci flow spacetime, we say it has normalized initial condition if it starts from a manifold $(M, g)$ with normalized geometry.

Definition 2.9 (Curvature scale). Let $(M, g)$ be a 3 dimensional Riemannian manifold and $x \in M$ a point. Let the curvature scale at $x$ be

$$
\rho(x)=R_{+}^{-1 / 2},
$$

where $R_{+}=\max \{R, 0\}$, and we use the convention $0^{-1 / 2}=\infty$.

Definition 2.10. (0-complete) We say a Ricci flow spacetime $\mathcal{M}$ is 0 -complete if for any smooth curve $\gamma:\left[0, s_{0}\right) \rightarrow \mathcal{M}$ that satisfies $\inf _{\left[0, s_{0}\right)} \rho(\gamma(s))>0$ and one of the following

(1) $\gamma\left(\left[0, s_{0}\right)\right)$ is contained in a time-slice $\mathcal{M}_{t}$, and has finite length with respect to the horizontal metric in $\mathcal{M}_{t}$, or

(2) $\gamma$ is the integral curve of $-\partial_{t}$, or $\partial_{t}$.

Then $\lim _{s \rightarrow s_{0}} \gamma(s)$ exists.

Also, we say a spacetime is backward (resp. forward) 0-complete if in case (2), $\gamma$ is only the integral curve of $-\partial_{t}\left(\right.$ resp. $\left.\partial_{t}\right)$.

We say a manifold is 0 -complete if it satisfies condition (1). 


\subsection{Singular Ricci flow.}

Definition 2.11 ( $\kappa$-solution). An ancient Ricci flow $\left(M, g(t)_{t \in(\infty, 0]}\right)$ on a 3 dimensional manifold $M$ is called a $\kappa$-solution if it satisfies the following:

(1) $(M, g(t))$ is complete for all $t \in(-\infty, 0]$,

(2) $|\mathrm{Rm}|$ is bounded on $M \times(-\infty, 0]$,

(3) sec $\geq 0$ on $M \times(-\infty, 0]$,

(4) $(M, g(t))$ is $\kappa$-non-collapsed at all scales for all $t \in(-\infty, 0]$.

Definition 2.12 (Geometric closeness). We say that a pointed Riemannian manifold $(M, g, x)$ is $\epsilon$-close to another pointed Riemannian manifold $(\bar{M}, \bar{g}, \bar{x})$ at scale $\lambda>0$ if there is a diffeomorphism onto its image

$$
\psi: B^{\bar{M}}\left(\bar{x}, \epsilon^{-1}\right) \rightarrow M
$$

such that $\psi(\bar{x})=x$ and

$$
\left\|\lambda^{-2} \psi^{*} g-\bar{g}\right\|_{C^{\left[\epsilon^{-1}\right]}\left(B^{\bar{M}}\left(\bar{x}, \epsilon^{-1}\right)\right)}<\epsilon .
$$

Here the $C^{\left[\epsilon^{-1}\right]}$-norm of a tensor $h$ is defined to be the sum of the $C^{0}$-norms of the tensors $h, \nabla^{\bar{g}} h, \nabla^{\bar{g}, 2} h, \ldots, \nabla^{\bar{g},\left[\epsilon^{-1}\right]} h$ with respect to the metric $\bar{g}$.

Similarly, we say a pointed Ricci flow $(M, g(t),(x, 0))$ is $\epsilon$-close to a pointed Ricci flow $(\bar{M}, \bar{g}(t),(\bar{x}, 0))$ on $[a, b](a \leq 0 \leq b)$ at scale $\lambda>0$ if $g(t)$ is defined on $\left[\lambda^{2} a, \lambda^{2} b\right]$, and there is a diffeomorphism onto its image

$$
\psi: B_{\bar{g}(0)}^{\bar{M}}\left(\bar{x}, \epsilon^{-1}\right) \rightarrow M
$$

such that $\psi(\bar{x})=x$ and

$$
\left\|\lambda^{-2} \psi^{*} g\left(\lambda^{2} t\right)-\bar{g}(t)\right\|_{\left.C \epsilon^{\left[\epsilon^{-1}\right](B \bar{g}(0)}\left(\bar{x}, \epsilon^{-1}\right)\right)}<\epsilon
$$

for all $t \in[a, b]$, where the norm is measured with respect to the metric $\bar{g}(t)$. In particular, when $a=-\epsilon^{-1}$ and $b=0$, we simply say $(M, g(t),(x, 0))$ is $\epsilon$-close to $(\bar{M}, \bar{g}(t),(\bar{x}, 0))$.

Definition 2.13 ( $\delta$-neck and strong $\delta$-neck). Let $(M, g)$ be a 3 dimensional Riemannian manifold and $\delta>0$. Suppose $U \subset M$ is an open subset, $x \in U$. We say $U$ is a $\delta$-neck centered at $x$, if $(U, g)$ is $\delta$-close to the standard cylindrical metric on $\left(-\delta^{-1}, \delta^{-1}\right) \times S^{2}$ at scale $\rho(x)$.

Let $(M, g(t))$ be a Ricci flow. Suppose $U \subset M$ is an open subset and $x$ is a point in $U$. We say that $U$ is a strong $\delta$-neck on $[-c, 0]$ centered at $x$ for some $c>0$, if $(U, g(t), x)$ is $\delta$-close to the standard cylindrical flow on the time interval $[-c, 0]$ at scale $\rho(x)$. We simply call it a strong $\delta$-neck when $c=-\delta^{-1}$.

Definition 2.14 (Canonical neighborhood assumption). Let $(M, g)$ be a 3 dimensional Riemannian manifold and $\epsilon>0$. We say that $(M, g)$ satisfies the $\epsilon$-canonical neighborhood assumption at some point $x \in M$ if there is a $\kappa$-solution $\left(\bar{M}, \bar{g}(t)_{t \in(-\infty, 0]}\right)$ and a point $\bar{x} \in \bar{M}$ such that $\rho(\bar{x}, 0)=1$ and $(M, g, x)$ is $\epsilon$-close to $(\bar{M}, \bar{g}(0), \bar{x})$ at scale $\rho(x)>0$. 
We say that $(M, g)$ satisfies the $\epsilon$-canonical neighborhood assumption at scales $\left(r_{1}, r_{2}\right)$, for some $r_{2}>r_{1}>0$, if $M$ satisfies the $\epsilon$-canonical neighborhood assumption at every point $x \in M$ with $r_{1}<\rho(x)<r_{2}$.

Lemma 2.15. (Gradient estimate, see e.g. [BK17b, Lemma 8.1]) There exist $\bar{\epsilon}$, and $\eta>0$ such that for all $\epsilon \leq \bar{\epsilon}$ the following holds: If $\mathcal{M}$ is a Ricci flow spacetime satisfying the $\epsilon$-canonical neighborhood assumption at some point $x \in \mathcal{M}$, then

$$
|\nabla \rho|(x) \leq \eta, \quad\left|\partial_{t} \rho^{2}(x)\right| \leq \eta
$$

Hereafter, we always assume $\epsilon>0$ to be smaller than the $\bar{\epsilon}$ from the above lemma whenever we talk about the $\epsilon$-canonical neighborhood assumption.

Lemma 2.16. ([BK17b, Lemma 8.2]) For every $\delta>0$ there are constants $C_{0}(\delta), \epsilon_{\text {can }}(\delta)>$ 0 such that if $\epsilon \leq \epsilon_{\text {can }}(\delta)$, then the following holds.

Let $(M, g)$ be a Riemannian manifold that satisfies the $\epsilon$-canonical neighborhood assumption at some point $x \in M$. Then $x$ is contained in a compact, connected domain $V \subset M$ such that $\operatorname{diam}(V) \leq C_{0} \rho(x)$ and $\rho\left(y_{1}\right) \leq C_{0} \rho\left(y_{2}\right)$ for all $y_{1}, y_{2} \in V$, and one of the following hold:

(1) $V$ is a $\delta$-neck at scale $\rho(x)$ and $x$ is its center.

(2) $V$ is a closed manifold without boundary.

(3) Either $V$ is a 3-disk or is diffeomorphic to a twisted interval bundle over $\mathbb{R} P^{2}$ and $\partial V$ is a central 2-sphere of a $\delta$-neck. We call $V$ a $\delta$-cap and $x$ its center. Moreover, for any $y_{1}, y_{2} \in \partial V$, we have $d\left(y_{1}, x\right)+d\left(y_{2}, x\right) \geq d\left(y_{1}, y_{2}\right)+100 \rho(x)$.

Definition 2.17 ( $\delta$-tube and capped $\delta$-tube). A $\delta$-tube $T$ in a Riemannian 3-manifold $M$ is a submanifold diffeomorphic to $S^{2} \times \mathbb{R}$ which is a union of $\delta$-necks with the central spheres that are isotopic to the 2-spheres of the product structure.

A capped $\delta$-tube is a connected submanifold that is the union of a $\delta$-cap and a $\delta$-tube where the intersection of them is diffeomorphic to $S^{2} \times \mathbb{R}$ and contains an end of the $\delta$-tube and an end of the $\delta$-cap.

Lemma 2.18. (High curvature regions are covered by tubes and capped-tubes) For some sufficiently small $\delta>0$, there exist $\epsilon_{\text {can }}(\delta), C_{0}(\delta), \lambda(\delta), \Lambda(\delta)>0$ such that the following holds:

Let $\left(M, g, x_{0}\right)$ be a 3 dimensional Riemannian manifold which is 0 -complete, $x_{0} \in$ $M, \rho\left(x_{0}\right) \geq C_{0}$. Suppose the $\epsilon_{\text {can }}$-canonical neighborhood assumption holds at scales $(0,1)$ on $B_{g}\left(x_{0}, d\right)$ for some $d \geq 2$. Let $r_{0} \in(0,1)$, then there exists a collection $S$ of disjoint $\delta$-tubes and capped $\delta$-tubes in $B_{g}\left(x_{0}, d\right)$ such that

(1) For all $x \in B_{g}\left(x_{0}, d-1\right)$ with $\rho(x) \leq \lambda r_{0}, x$ is contained in $\bigcup_{V \in S} V$.

(2) $\rho \leq \Lambda r_{0}$ on $\bigcup_{V \in S} V$.

(3) The boundary components of all $V \in S$ are central spheres of some $\delta$-neck with scale equal to $r_{0}$.

(4) Suppose for some $C>0, \operatorname{vol}\left(B_{g}\left(x_{0}, d\right)\right) \leq C$. Then there is $N\left(C, r_{0}\right)>0$ such that the number of elements in $S$ is less than $N$. 
Proof of Lemma 2.18. Let $x \in B_{g}\left(x_{0}, d\right)$ with $\rho(x)<1$. Then by $\rho\left(x_{0}\right) \geq C_{0}$ and Lemma 2.16, $x$ must be the center of a $\delta$-neck or a $\delta$-cap. Note also that by [MT09, Proposition 19.21], a connected subset consisting of points which are centers of a $\delta$-neck or a $\delta$-cap is a $\delta$-tube or a capped $\delta$-tube. Now the proof is an easy adaptation of [BK17a, Lemma 9.4].

Definition 2.19 (Singular Ricci flow). If $\epsilon>0$ and $r(t):[0, \infty) \rightarrow(0, \infty)$ is a nonincreasing function. Then we say a Ricci flow space time $\mathcal{M}$ is an $(r, \epsilon)$-singular Ricci flow if the following holds:

(1) $\mathcal{M}_{0}$ is a compact orientable manifold;

(2) $\mathcal{M}$ is 0-complete;

(3) $\mathcal{M}_{[0, t]}$ satisfies the $\epsilon$-canonical neighborhood assumption at scales $(0, r(t))$.

We call $t$ a singular time if $\mathcal{M}_{t}$ is not compact.

It was shown in [KL17, Theorem 1.3] that for any 3 dimensional compact Riemannian manifold $(M, g)$, there exists an $(r, \epsilon)$-singular Ricci flow with $\mathcal{M}_{0}$ isometric to $(M, g)$.

\subsection{Distance distortion estimates.}

In this subsection, we review some standard distance distortion estimates under different curvature conditions. which are originally due to Hamilton and Perelman.

Lemma 2.20. (see e.g. $\left[\mathrm{CCG}^{+} 10\right.$, Theorem 18.7]) Let $(M, g(t))_{t \in[0, T]}$ be a Ricci flow of dimension $n$. Let $K, r_{0}>0$.

(1) Let $x_{0} \in M$ and $t_{0} \in(0, T)$. Suppose that Ric $\leq(n-1) K$ on $B_{t_{0}}\left(x_{0}, r_{0}\right)$. Then the distance function $d(x, t)=d_{t}\left(x, x_{0}\right)$ satisfies

$$
\left.\left(\partial_{t}-\Delta\right)\right|_{t=t_{0}} d \geq-(n-1)\left(\frac{2}{3} K r_{0}+r_{0}^{-1}\right) .
$$

(2) Let $t_{0} \in[0, T)$ and $x_{0}, x_{1} \in M$. Suppose

$$
\operatorname{Ric}\left(x, t_{0}\right) \leq(n-1) K,
$$

for all $x \in B_{t_{0}}\left(x_{0}, r_{0}\right) \cup B_{t_{0}}\left(x_{1}, r_{0}\right)$. Then

$$
\left.\partial_{t}\right|_{t=t_{0}} d_{t}\left(x_{0}, x_{1}\right) \geq-2(n-1)\left(K r_{0}+r_{0}^{-1}\right) .
$$

Lemma 2.21. (see e.g. [ST17, Lemma 2.1, 2.2]) Let $(M, g(t))_{t \in[0, T]}$ be a Ricci flow of dimension $n, x_{0} \in M$. Let $K, A>0$.

(1) Suppose $\operatorname{Ric}_{g(t)} \geq-K$ on $B_{t}\left(x_{0}, A\right) \subset \subset M$ for all $t \in[0, T]$. Then the following holds for all $t \in[0, T]$ :

$$
B_{0}\left(x_{0}, R e^{-K T}\right) \subset B_{t}\left(x_{0}, R e^{-K(T-t)}\right) .
$$

(2) Suppose $\operatorname{Ric}_{g(t)} \leq K$ on $B_{t}\left(x_{0}, R\right) \subset \subset M$ for all $t \in[0, T]$. Then the following holds for all $t \in[0, T]$ :

$$
B_{t}\left(x_{0}, R e^{-K t}\right) \subset B_{0}\left(x_{0}, R\right) .
$$




\section{Preparatory Results}

In this section we prove some technical lemmas that will be used later. First, in Lemma 3.1 3.3, we study manifolds that are 0-complete and satisfy a canonical neighborhood assumption at scales depending on the distance to a base point $x_{0}$. We show for such manifolds that any metric ball of a fixed radius centered at $x_{0}$ is uniformly totally bounded (Lemma 3.1), and for any point $x \in M$, there exists a minimizing geodesic from $x_{0}$ to $x$ (Lemma 3.2).

Second, we show in Lemma 3.4 that for a Ricci flow spacetime with some appropriate canonical neighborhood assumption, the closeness of a time-slice to a $\kappa$-solution implies the closeness in a parabolic region. As a consequence, Lemma 3.5 shows that a blow-up sequence in a singular Ricci flow converges to a $\kappa$-solution defined from $-\infty$ to its maximal existence time.

Lemma 3.1. (Metric balls are uniformly totally bounded) Let $\left(M, g, x_{0}\right)$ be a 3 dimensional connected Riemannian manifold, $x_{0} \in M$. Suppose $M$ is 0-complete and 1-positive. Suppose for any $A, \epsilon_{\text {can }}>0$, there are $r\left(A, \epsilon_{\text {can }}\right), \kappa(A)>0$ such that the $\epsilon_{\text {can }}$-canonical neighborhood assumption and the $\kappa(A)$-non-collapsing assumption hold at scales less than $r\left(A, \epsilon_{\text {can }}\right)$ on $B_{g}\left(x_{0}, A\right)$.

Then for any given $A, \epsilon>0$, there exist $V(A), N(A, \epsilon)>0$ such that $\operatorname{vol}\left(B_{g}\left(x_{0}, A\right)\right) \leq$ $V(A)$, and the number of elements in any $\epsilon$-separating subset of $B_{g}\left(x_{0}, A\right)$ is bounded above by $N(A, \epsilon)$.

Proof. Fix some small $\delta>0$ and let $\epsilon_{\text {can }}(\delta), C_{0}(\delta)>0$ be from Lemma 2.16, Let $\eta>0$ be from Lemma 2.15. Let $r_{0}=\frac{1}{7 \eta} \cdot \min \left\{r\left(A+1, \epsilon_{\text {can }}\right), \frac{1}{6}\right\}$.

We claim that there exists a universal constant $C_{1}>0$ such that $\operatorname{vol}\left(B_{g}\left(x, 3 r_{0}\right)\right) \leq$ $C_{1}$ for all $x \in B_{g}\left(x_{0}, A\right)$. In fact, suppose first that $\rho(x)<4 \eta r_{0}$, then by Lemma 2.15 we get $\rho<7 \eta r_{0}$ on $B_{g}\left(x, 3 r_{0}\right)$. By the assumption of $r_{0}$, we see that $B_{g}\left(x, 3 r_{0}\right)$ is contained in some $\delta$-tube or capped $\delta$-tube with diameter less than 1 , which has volume less than $C_{1}$. So the claim holds. Next, suppose $\rho(x) \geq 4 \eta r_{0}$, then by Lemma 2.15 we get $\rho \geq \eta r_{0}$ on $B_{g}\left(x, 3 r_{0}\right)$. So the claim follows from the Bishop-Gromov volume comparison.

Now suppose by induction that for some $k \in \mathbb{N}$ with $k r_{0} \leq A$, there exist $C_{k}(A)>0$ and $N_{k}(A, \epsilon) \in \mathbb{N}_{+}$such that the following holds:

$\mathcal{A}(\mathrm{k}): \operatorname{vol}\left(B_{g}\left(x_{0}, k r_{0}\right)\right) \leq C_{k}$

$\mathcal{B}(\mathrm{k})$ : For any $\epsilon \leq r_{0}$, an $\epsilon$-separating subset in $B_{g}\left(x_{0},(k-1) r_{0}\right)$ has at most $N_{k}$ elements.

To show $\mathcal{A}(k+1)$, consider a maximal $r_{0}$-separating subset $\left\{y_{j}\right\}$ in $B_{g}\left(x_{0},(k-1) r_{0}\right)$. Then $B_{g}\left(x_{0},(k-1) r_{0}\right)$ is covered by the union of all $B_{g}\left(y_{j}, r_{0}\right)$, and by the triangle inequality we get

$$
B_{g}\left(x_{0},(k+1) r_{0}\right) \subset \bigcup_{j} B_{g}\left(y_{j}, 3 r_{0}\right)
$$


So $\mathcal{A}(\mathrm{k}+1)$ follows from the above claim and $\mathcal{B}(\mathrm{k})$. It remains to establish $\mathcal{B}(k+1)$.

Let $\left\{x_{j}\right\}_{j=1}^{m}$ be an $\epsilon$-separating set in $B_{g}\left(x_{0}, k r_{0}\right)$. First, by the non-collapsing assumption and $\mathcal{A}(k+1)$ we see that the number of $x_{j}$ with $\rho\left(x_{j}\right) \geq\left(2 C_{0}\right)^{-1} \epsilon$ is bounded above in terms of $A, \epsilon$. So we may assume $\rho\left(x_{j}\right)<\left(2 C_{0}\right)^{-1} \epsilon$ for all $j$. Then by $\mathcal{A}(k+1)$ and Lemma 2.18 , we may further assume that all $x_{j}$ are contained in a single $\delta$-tube or capped $\delta$-tube $V \subset B_{g}\left(x_{0},(k+1) r_{0}\right)$.

Pick a point $y \in \partial V$, we can arrange the order of $\left\{x_{j}\right\}_{j=1}^{m}$ in a way such that $d_{V}\left(y, x_{j+1}\right) \geq d_{V}\left(y, x_{j}\right)$ for each $j \leq m-1$. Here $d_{V}$ denotes the length metric in $V$ induced by $g$. We claim that each $x_{j}, j \leq m-1$, is the center of a $\delta$-neck. Otherwise, $x_{j}$ is the center of a $\delta$-cap $\mathcal{C} \subset V$. By Lemma 2.16 we have $\operatorname{diam}(\mathcal{C}) \leq C_{0} \rho\left(x_{j}\right) \leq \epsilon / 2$. Since $d_{V}\left(x_{j}, x_{j+1}\right) \geq \epsilon$, we get $x_{j+1} \in V-\mathcal{C}$, and $x_{j+1}$ is the center of a $\delta$-neck. Connecting $y$ with $x_{j}$ by a minimizing geodesic, then it must intersect the central sphere at $x_{j+1}$, which has diameter less than $10\left(2 C_{0}\right)^{-1} \epsilon<\epsilon / 2$. So it is easy to see $d_{V}\left(y, x_{j+1}\right)<d_{V}\left(y, x_{j}\right)$, a contradiction.

So by the triangle inequality we get

$$
d_{V}\left(y, x_{j+1}\right) \geq d_{V}\left(y, x_{j}\right)+\epsilon-2 \cdot 10 \cdot\left(2 C_{0}\right)^{-1} \epsilon \geq d_{V}\left(y, x_{j}\right)+\epsilon / 2,
$$

for all $j \leq m-1$. In particular, this implies

$$
(m-1) \epsilon / 2 \leq d_{V}\left(x_{m}, y\right) \leq \operatorname{diam}(V) \leq 2(k+1) r_{0},
$$

and hence $m \leq 4(k+1) r_{0} \epsilon^{-1}+1$. This established $\mathcal{B}(k+1)$.

Lemma 3.2. Under the same assumptions as Lemma 3.1. Then for any $x \in M$, there exists a minimizing geodesic connecting $x$ to $x_{0}$.

Proof. By Lemma 3.1 we have $\operatorname{vol}\left(B_{g}\left(x_{0}, A\right)\right)$ is bounded above, so by Lemma 2.15 it is easy to see that $|\mathrm{Rm}|$ is a proper function restricted on $\overline{B_{g}\left(x_{0}, A\right)}$ for all $A>0$. Suppose $d=d_{g}\left(x_{0}, x\right)>0$. Fix a sufficiently small number $\delta>0$, and let $\epsilon_{\text {can }}=$ $\epsilon_{c a n}(\delta), C_{0}=C_{0}(\delta)$ be from Lemma 2.16. Let $r_{0}=\min \left\{C_{0}^{-1} \rho\left(x_{0}\right), C_{0}^{-1} \rho(x), r(d+\right.$ $\left.\left.2, \epsilon_{\text {can }}\right), 1\right\}$. Then by Lemma 2.18 and Lemma 3.1, all points in $B_{g}\left(x_{0}, d+1\right)$ with $\rho \leq r_{0}$ are contained in the union of a finite collection $S$ of disjoint $\delta$-tubes and capped $\delta$-tubes in $B_{g}\left(x_{0}, d+2\right)$.

For each $i \in \mathbb{N}$, let $\gamma_{i}:[0, d] \rightarrow M$ be a smooth curve joining $x_{0}$ and $x$ with constant speed, such that the length of $\gamma_{i}$ satisfies $L\left(\gamma_{i}\right)<d+\frac{1}{i}$. So $\gamma_{i} \subset B_{g}\left(x_{0}, d+1\right)$. We claim that the curvature on $\gamma_{i}$ is uniformly bounded for all $i$. Suppose not, we may assume there is a sequence of points $x_{i} \in \gamma_{i}$ such that $\rho\left(x_{i}\right) \rightarrow 0$ as $i \rightarrow \infty$. By the finiteness of $S$ we may also assume there is some $\mathcal{T} \in S$ that contains all $x_{i}$. Then $\mathcal{T}$ must be a $\delta$-tube with curvature blowing up in one end. Taking a point $y \in \mathcal{T}$ such that $\rho(y)<\frac{1}{2} \min \left\{\rho\left(x_{0}\right), \rho(x)\right\}$. Then for all large $i, \gamma_{i}$ passes through the $\delta$-neck centered at $y$ at least twice, which contradicts the almost minimality of $\gamma_{i}$. So the claim holds. Therefore, by the properness of $|\mathrm{Rm}|$, there is a compact set $K \subset M$ such that $\gamma_{i} \subset K$ for all $i$. 
Since $\left|\gamma_{i}^{\prime}\right|=\frac{L\left(\gamma_{i}\right)}{d} \rightarrow 1$, it follows that $\gamma_{i}^{\prime}$ 's are uniformly Lipschiz-continuous on $[0, d]$. Since $\gamma_{i}^{\prime}$ 's are equicontinuous and map into a compact set of $M$, the ArzelaAscoli Lemma applies. So by passing to a subsequence, we may assume that $\gamma_{i}$ uniformly converges to some continuous curve $\gamma_{\infty}:[0, d] \rightarrow M$. Since $\int_{0}^{d}\left|\gamma_{i}^{\prime}\right|^{2} d t=$ $\frac{L\left(\gamma_{i}\right)^{2}}{d} \leq 4 d$, we can apply weak compactness to the sequence $\left\{\gamma_{i}\right\}$. By passing to a subsequence, we may assume $\gamma_{i}$ weakly converges to $\gamma_{\infty}$ in $W^{1,2}$.

Let $E(\gamma)=\int_{0}^{d}\left|\gamma^{\prime}(t)\right|^{2} d t$ be the energy function on all $W^{1,2}$-path connecting $x_{0}$ and $x$. Then by Cauchy-Schwarz inequality we have

$$
E(\gamma)=\int_{0}^{d}\left|\gamma^{\prime}(t)\right|^{2} d t \geq \frac{\left(\int_{0}^{d}\left|\gamma^{\prime}\right|\right)^{2}}{d}=\frac{L(\gamma)^{2}}{d} \geq d
$$

By the weak semi-continuity of the $E$-energy, it follows that the $W^{1,2}$-path $\gamma_{\infty}$ has energy $E\left(\gamma_{\infty}\right) \leq d$. So $\gamma_{\infty}$ minimizes the energy $E$ in $W^{1,2}$. Therefore, $\gamma_{\infty}$ is a smooth solution to the geodesic equation, and hence it is a minimizing geodesic.

The next lemma says that if a ball is scathed, then we can find a minimizing geodesic in the ball along which the curvature blows up, and it is covered by $\delta$-necks.

Lemma 3.3. (Minimal geodesic covered by $\delta$-necks) Under the same assumptions as Lemma 3.1. Let $\delta>0$. Suppose $\inf _{B_{g}\left(x_{0}, A\right)} \rho=0$ for some $A>0$. Then there exists a minimizing geodesic $\gamma:[0,1) \rightarrow B_{g}\left(x_{0}, A\right)$ such that $R(\gamma(s)) \rightarrow \infty$ as $s \rightarrow 1$, and $\gamma(s)$ is the center of a $\delta$-neck for all s close to 1 .

Proof. Let $x_{i} \in B_{g}\left(x_{0}, A\right)$ be a sequence of points such that $R\left(x_{i}\right) \rightarrow \infty$ as $i \rightarrow \infty$. Since by Lemma 3.1 the ball $B_{g}\left(x_{0}, A\right)$ is totally bounded, we may assume by passing to a subsequence that $\left\{x_{i}\right\}$ is Cauchy. So there is a $\delta$-tube $\mathcal{T}$, which blows up at one end, that contains $x_{i}$ for all large $i$. By Lemma 3.2 , there exists $\gamma_{i}:[0,1] \rightarrow M$, which is the minimizing geodesic connecting $x_{0}$ and $x_{i}$. Noting that $\gamma_{i}$ passes through any $\delta$-neck in $\mathcal{T}$ at most once, after passing to a subsequence, $\gamma_{i}$ converges to a minimizing geodesic $\gamma:[0,1) \rightarrow M$. Moreover, by the minimality of $\gamma, \gamma(s)$ is the center of a $\delta$-neck for $s$ close enough to 1 .

The following lemma shows that for a Ricci flow spacetime satisfying some appropriate canonical neighborhood assumption, suppose a time-slice is close enough to that of a $\kappa$-solution, then a parabolic region of a certain size is close to that in the $\kappa$-solution.

Lemma 3.4. (Time-slice closeness implies spacetime closeness) Let $\left(M_{\infty}, g_{\infty}(t), x_{\infty}\right)$ be a $\kappa$-solution, and $a, b>0$ be constants such that $g_{\infty}(t)$ is defined for all $t \in[-a, b]$. Let $\delta>0$. Then there exists $\epsilon>0$ such that the following holds: 
Let $\mathcal{M}$ be a Ricci flow spacetime, $x_{0} \in \mathcal{M}, t_{0}:=\mathfrak{t}\left(x_{0}\right)$. Suppose $\left(\mathcal{M}_{t_{0}}, x_{0}\right)$ is $\epsilon$-close to $\left(M_{\infty}, g_{\infty}(0), x_{\infty}\right)$. Suppose also that $B_{t_{0}}\left(x_{0}, \epsilon^{-1}\right)$ survives on $\left[t_{0}-a, t_{0}+b\right]$, and the $\epsilon$ canonical neighborhood assumption holds at every point in $\bigcup_{t \in\left[t_{0}-a, t_{0}+b\right]}\left(B_{t_{0}}\left(x_{0}, \epsilon^{-1}\right)\right)(t)$.

Then $\left(\mathcal{M}, x_{0}\right)$ is $\delta$-close to $\left(M_{\infty}, g_{\infty}(t), x_{\infty}\right)$ on the time interval $[-a, b]$.

Proof. Suppose the assertion does not hold, then there is a sequence of spacetimes $\mathcal{M}_{i}$ and points $x_{0 i} \in \mathcal{M}_{i}$, and a sequence of $\epsilon_{i}>0$ with $\lim _{i \rightarrow \infty} \epsilon_{i}=0$, such that the assumptions are satisfied for each $i$, but the conclusion fails.

Let $-a^{*}, b^{*}$ be the infimum and supremum of $s_{1}, s_{2} \in[-a, b]$, respectively, such that there exists $C$ (may depend on $s_{1}, s_{2}$ ) such that for all $d>0,|\operatorname{Rm}| \leq C$ in $\bigcup_{t \in\left[t_{0 i}-s_{1}, t_{0 i}+s_{2}\right]}\left(B_{t_{0 i}}\left(x_{0 i}, d\right)\right)(t)$. Then by the gradient estimate we have $a^{*}, b^{*}>0$. So by passing to a subsequence, we may assume $\left(\mathcal{M}_{i}, x_{0 i}\right)$ converges to a smooth complete Ricci flow $(\widehat{M}, \widehat{g}(t), \widehat{x}), t \in\left(-a^{*}, b^{*}\right)$, which has bounded curvature in any compact subinterval in $\left(-a^{*}, b^{*}\right)$.

Note that $(\widehat{M}, \widehat{g}(0), \widehat{x})$ is isometric to $\left(M_{\infty}, g_{\infty}(0), x_{\infty}\right)$, and each time-slice of $(\widehat{M}, \widehat{g}(t))$ is a time-slice of a $\kappa$-solution, it is easy to see that the flow $(\widehat{M}, \widehat{g}(t), \widehat{x})$ is isometric to $\left(M_{\infty}, g_{\infty}(t), x_{\infty}\right)$ for all $t \in\left(-a^{*}, b^{*}\right)$.

So $(\widehat{M}, \widehat{g}(t), \widehat{x})$ extends to a complete Ricci flow with bounded curvature on $\left[-a^{*}, b^{*}\right]$. Applying the gradient estimates on $\left(B_{t_{0 i}}\left(x_{0 i}, \epsilon^{-1}\right)\right)(t)$ for $t=t_{0 i}-a^{*}$ and $t_{0 i}+b^{*}$, and seeing the assumptions of $a^{*}$ and $b^{*}$, we get $a^{*}=a$ and $b^{*}=b$. From this it follows that $\left(\mathcal{M}_{i}, x_{0 i}\right)$ is $\delta$-close to $\left(M_{\infty}, g_{\infty}(t), x_{\infty}\right)$ on $[-a, b]$, which is a contradiction.

Lemma 3.5. (Blow-up sequence converges to a $\kappa$-solution) Let $\mathcal{M}$ be a singular Ricci flow with normalized initial condition. Let $x_{i} \in M$ be a sequence of points such that $\sup _{i} \mathfrak{t}\left(x_{i}\right)<\infty$, and $\sup _{i}|\operatorname{Rm}|\left(x_{i}\right)=\infty$. Then there exists a subsequence $\left\{x_{i_{k}}\right\}$, such that $\left(\mathcal{M}, x_{i_{k}}\right)$ converges to a $\kappa$-solution on its maximal existence time interval.

Proof. By [KL17, Theorem 1.3], the canonical neighborhood assumption for singular Ricci flows, and the gradient estimates, the conditions in Lemma 3.4 are satisfied. Assume $\left(\mathcal{M}_{\mathfrak{t}\left(x_{i}\right)}, x_{i}\right)$ converges to the time-0-slice of a $\kappa$-solution $\left(M_{\infty}, g_{\infty}(t), x_{\infty}\right)$. Then by Lemma 3.4, after passing to a subsequence, $\left(\mathcal{M}, x_{i_{k}}\right)$ converges to a $\kappa$ solution on its maximal existence time interval.

\section{CAnonical neighborhood theOREM}

The main results in this section are a canonical neighborhood theorem (Proposition 4.1) for singular Ricci flows, and a bounded curvature at bounded distance theorem by reduced volume (Theorem 4.2). 
Recall that Perelman proved a canonical neighborhood theorem for compact smooth Ricci flows [KL08, Theorem 26.2], which says that assuming the Ricci flow has normalized initial condition, then for any $T>0$ there exists $r(T) \geq 0$ such that the canonical neighborhood assumption holds in $\mathcal{M}_{t \leq T}$ at scales less than $r(T)$.

He also proved a local version of the theorem [KL08, Proposition 85.1], in which he assumed the curvature is bounded in a backward parabolic neighborhood of a point $x_{0}, \mathfrak{t}\left(x_{0}\right)=t_{0}$, and showed that for all $A>0$ there exists $r(A)>0$, such that the canonical neighborhood assumption holds in $B_{t_{0}}\left(x_{0}, A\right)$ at scales less than $r(A)$.

The following proposition extends the local theorem to singular Ricci flows.

Proposition 4.1. (Canonical neighborhood theorem) For any $A>0, \epsilon>0$, there are constants $\kappa(A), r(A, \epsilon), \bar{r}(A), K(A)>0$, such that the following holds: Let $\mathcal{M}$ be a singular Ricci flow, $x_{0} \in \mathcal{M}, \mathfrak{t}\left(x_{0}\right)=t_{0}>0$. Suppose for some $r_{0}>0$ with $2 r_{0}^{2}<t_{0}$ the following holds:

(1) $\mathcal{M}$ is unscathed on a parabolic neighborhood $P\left(x_{0}, r_{0},-r_{0}^{2}\right)$.

(2) $|\mathrm{Rm}| \leq r_{0}^{-2}$ on $P\left(x_{0}, r_{0},-r_{0}^{2}\right)$.

(3) $\operatorname{vol}\left(B_{t_{0}}\left(x_{0}, r_{0}\right)\right) \geq A^{-1} r_{0}^{3}$.

Then

(a) The solution is $\kappa$-non-collapsed at scales less than $r_{0}$ in $B_{t_{0}}\left(x_{0}, A r_{0}\right)$.

(b) The $\epsilon$-canonical neighborhood assumption holds in $B_{t_{0}}\left(x_{0}, A r_{0}\right)$ at scales less than $\mathrm{rr}_{0}$.

(c) If $r_{0} \leq \bar{r} \sqrt{t_{0}}$ then $|\mathrm{Rm}| \leq K r_{0}^{-2}$ in $B_{t_{0}}\left(x_{0}, A r_{0}\right)$.

As an application of Proposition 4.1, as well as other results by Perelman, we can generalize his bounded curvature at bounded distance theorem by using the reduced volume:

Theorem 4.2. (Bounded curvature at bounded distance by reduced volume) For any $A, \kappa>0$, there exist $\varphi(A, \kappa), K(A, \kappa)>0$ such that the following holds: Let $\mathcal{M}$ be a singular Ricci flow, $x_{0} \in \mathcal{M}, t_{0}:=\mathfrak{t}\left(x_{0}\right)$. Suppose the reduced volume $\tilde{V}_{x_{0}}(1) \geq \kappa$, and $\mathcal{M}_{t \geq t_{0}-1}$ is $\varphi$-positive.

If $|\operatorname{Rm}|\left(x_{0}\right) \leq 1$, then $|\mathrm{Rm}| \leq K$ in $B_{t_{0}}\left(x_{0}, A\right)$.

In the theorem, $\tilde{V}_{x_{0}}(1)$ is the reduced volume at $\tau=1$, for the base point $x_{0}$. It is defined by

$$
\tilde{V}_{x_{0}}(\tau)=\int_{\mathcal{M}_{t_{0}-\tau}} \tau^{-\frac{3}{2}} e^{-\ell_{x_{0}}(x)} d_{t_{0}-\tau} x .
$$

To prove Proposition 4.1 and Theorem 4.2, we first prove some results in $\mathcal{L}$ geometry for singular Ricci flows. 
Definition 4.3. The $\mathcal{L}_{+}$-length of an admissible curve $\gamma:\left[t_{0}-\tau, t_{0}\right] \rightarrow \mathcal{M}$ is

$$
\mathcal{L}_{+}(\gamma)=\int_{t_{0}-\tau}^{t_{0}} \sqrt{t_{0}-t}\left(R_{+}(\gamma(t))+\left|\gamma^{\prime}(t)\right|^{2}\right) d t
$$

Lemma 4.4. Given constants $T, E$ and $\Lambda>0$, there exists $r=r(\Lambda, T, E)$ such that the following holds. Let $\mathcal{M}$ be a singular Ricci flow with normalized initial condition. Suppose $\gamma:[a, b] \rightarrow \mathcal{M}$ is a smooth admissible curve with $b \leq T$. Suppose $b-a \geq E$, and $\rho(\gamma(a)) \leq r$.

$$
\text { Then } \int_{a}^{b} R_{+}(\gamma(t))+\left|\gamma^{\prime}(t)\right|^{2} d t>\Lambda \text {. }
$$

Proof. Suppose for some $\Lambda, T, E>0$, the conclusion does not hold, then we can find a sequence of singular Ricci flows $\left(\mathcal{M}_{k}, g_{k}(t)\right)$ and a sequence of smooth curves $\gamma_{k}:\left[a_{k}, b_{k}\right] \rightarrow \mathcal{M}_{k}$ satisfying the assumptions in the theorem. In particular, we have $\rho\left(\gamma_{k}\left(a_{k}\right)\right) \leq r_{k}$, and $r_{k} \rightarrow 0$ as $k \rightarrow \infty$, but

$$
\int_{a_{k}}^{b_{k}}\left(R_{+}\left(\gamma_{k}(t)\right)+\left|\gamma_{k}^{\prime}(t)\right|^{2}\right) d t \leq \Lambda
$$

We rescale each $\left(\mathcal{M}_{k}, g_{k}(t), \gamma_{k}\left(a_{k}\right)\right)$ by $\rho_{k}^{-2}:=\rho^{-2}\left(\gamma_{k}\left(a_{k}\right)\right)$ and then shift time $a_{k}$ to 0 , to obtain a sequence of Ricci flow spacetimes $\left(\tilde{\mathcal{M}}_{k}, \tilde{g}_{k}(t), \gamma_{k}\left(a_{k}\right)\right)$ defined on $[0, \infty)$. Then $\gamma_{k}:\left[0,\left(b_{k}-a_{k}\right) \rho_{k}^{-2}\right] \rightarrow \tilde{\mathcal{M}}_{k}$ is an admissible curve and $\left(b_{k}-a_{k}\right) \rho_{k}^{-2} \rightarrow \infty$ as $k \rightarrow \infty$. Note that (4.3) is invariant under rescaling. Applying Lemma 3.5 and passing to a subsequence we may assume that $\left(\tilde{\mathcal{M}}_{k}, \tilde{g}_{k}(t), \gamma_{k}\left(a_{k}\right)\right)$ converges to a $\kappa$ solution $\left(M_{\infty}, g_{\infty}(t), x_{\infty}\right)$ on $[0, \infty)$. So by [Bre18], $\left(M_{\infty}, g_{\infty}(t)\right)$ is either a Bryant soliton [Bre18], or has finite extinction time.

Suppose first that $\left(M_{\infty}, g_{\infty}(t)\right)$ is a Bryant soliton. Let $\mathcal{M}_{\infty}$ denote the Ricci flow spacetime associated to it. Fix a large $A>0$ and let $\mathcal{P}_{A}:=\bigcup_{t \in\left[0, A^{3 / 2}\right]} B_{t}\left(x_{\infty}, A\right)$. Then for large $k$, there exists a time-preserving diffeomorphism $\phi_{k}: U_{k} \rightarrow V_{k}$, with $\phi_{k}\left(x_{\infty}\right)=\gamma_{k}\left(a_{k}\right)$, where $U_{k}$ and $V_{k}$ are open subsets of $\mathcal{M}_{\infty}$ and $\tilde{\mathcal{M}}_{k}$ respectively, such that given any compact subset $K \subset \mathcal{M}_{\infty}$ and $\delta>0$, we have $K \subset U_{k}$ for all large $k$, and $\left\|\phi_{k}^{*} G_{k}-G_{\infty}\right\|_{C^{[\delta-1]}\left(K, G_{\infty}\right)}$, where $G_{k}$ and $G_{\infty}$ are spacetime metrics of $\tilde{\mathcal{M}}_{k}$ and $\mathcal{M}_{\infty}$ respectively. So we have $\mathcal{P}_{A} \subset U_{k}$ for all large $k$. Let $\widehat{\gamma}_{k} \subset U_{k}$ be the image of $\left.\gamma_{k}\right|_{V_{k}}$ under $\phi_{k}^{-1}$.

Since $\left(b_{k}-a_{k}\right) \rho_{k}^{-2} \rightarrow \infty$ as $k \rightarrow \infty$, we see that $\gamma_{k}$ must exit $\phi_{k}\left(\mathcal{P}_{A}\right)$ at some time $T_{k} \in\left[0, A^{3 / 2}\right]$, and accordingly $\widehat{\gamma}_{k}$ exits $\mathcal{P}_{A}$ at the time $\widehat{T}_{k} \in\left[0, A^{3 / 2}\right]$. Then for sufficiently large $k$, we have

$$
\begin{aligned}
\int_{0}^{T_{k}}\left|\gamma_{k}^{\prime}(t)\right|^{2} d t & \geq \frac{1}{2} \int_{0}^{\widehat{T}_{k}}\left|\widehat{\gamma}_{k}^{\prime}(t)\right|^{2} d t, \\
\int_{0}^{T_{k}} R\left(\gamma_{k}(t)\right) d t & \geq \frac{1}{2} \int_{0}^{\widehat{T}_{k}} R\left(\widehat{\gamma_{k}}(t)\right) d t .
\end{aligned}
$$


Suppose $T_{k}<A^{3 / 2}$. Since Ric $\geq 0$, we have $\partial_{t} g_{\infty}(t) \leq 0$ for all $t \geq 0$. So $\left|\widehat{\gamma}_{k}^{\prime}(t)\right|_{g_{\infty}(t)} \geq\left|\widehat{\gamma}_{k}^{\prime}(t)\right|_{g_{\infty}\left(T_{k}\right)}$ for all $t \in\left[0, T_{k}\right]$, and hence

$$
\int_{0}^{T_{k}}\left|\widehat{\gamma}_{k}^{\prime}(t)\right|^{2} d t \geq \int_{0}^{T_{k}}\left|\widehat{\gamma}_{k}^{\prime}(t)\right|_{T_{k}}^{2} d t \geq \frac{d_{T_{k}}^{2}\left(\widehat{\gamma}_{k}(0), \widehat{\gamma}_{k}\left(T_{k}\right)\right)}{T_{k}} \geq A^{1 / 2}
$$

Otherwise, we have $T_{k}=A^{3 / 2}$, and $\widehat{\gamma}_{k}(t) \in B_{t}\left(\left(\widehat{\gamma}_{k}(0)\right)(t), A\right)$ for all $t \in\left[0, A^{3 / 2}\right]$. Since $\left(M_{\infty}, g_{\infty}(t)\right)$ is a Bryant soliton, we have $R\left(\widehat{\gamma}_{k}(t)\right) \geq \frac{C}{A}$, where $C$ is a constant depending only on the curvature of the tip. So we have

$$
\int_{0}^{A^{3 / 2}} R\left(\widehat{\gamma}_{k}(t)\right) d t \geq \int_{0}^{A^{3 / 2}} \frac{C}{A} d t=C A^{1 / 2} .
$$

In both cases, taking $A$ sufficiently large, it follows by (4.4) that

$$
\int_{0}^{T_{k}}\left(R\left(\gamma_{k}(t)\right)+\left|\gamma_{k}^{\prime}\right|^{2}\right) d t>\Lambda
$$

a contradiction to (4.3).

Now suppose $\left(M_{\infty}, g_{\infty}(t)\right)$ has a finite extinction time $T_{\infty}<\infty$. Let $\theta \in\left(0, T_{\infty}\right)$ and consider $\mathcal{P}_{A}:=\bigcup_{t=0}^{\theta} B_{t}\left(x_{\infty}, A\right)$. Let $T_{k}$ and $\widehat{T}_{k}$ be defined as above. Then if $\widehat{T}_{k}<\theta$, then (4.5) holds. Otherwise, since the scalar curvature blows up at the rate of $\left(T_{\infty}-t\right)^{-1}$ when the time goes up to $T_{\infty}$, we have

$$
\int_{0}^{\theta} R\left(\widehat{\gamma}_{k}(t)\right) d t \geq \int_{0}^{\theta} \frac{C}{T_{\infty}-t} d t=-C \log (T-\theta) .
$$

By taking $\theta$ sufficiently close to $T_{\infty}$ and $A$ sufficiently large, we get a contradiction.

The following lemma says that an admissible curve that contains a point of large curvature must have large $\mathcal{L}_{+}$-length.

Lemma 4.5. For all $\Lambda<\infty, \bar{r}, T>0$, there is a constant $\delta=\delta(\Lambda, \bar{r}, T)$ with the following property:

Let $\mathcal{M}$ be a singular Ricci flow with normalized initial condition, and $x_{0} \in \mathcal{M}_{t_{0}}$ with $0<t_{0} \leq T$. Suppose $r_{0} \geq \bar{r}$, and $P\left(x_{0}, r_{0},-r_{0}^{2}\right)$ is unscathed, and $|\mathrm{Rm}| \leq r_{0}^{-2}$ on $P\left(x_{0}, r_{0},-r_{0}^{2}\right)$. Suppose also $\gamma:\left[t_{1}, t_{0}\right] \rightarrow \mathcal{M}$ is an admissible curve ending at $\left(x_{0}, t_{0}\right)$, and there exists $t \in\left[t_{1}, t_{0}\right]$ such that $\rho(\gamma(t))<\delta$.

Then $\mathcal{L}_{+}(\gamma)>\Lambda$.

Proof. First, let $\Delta t=\frac{1}{4} 10^{-4} \bar{r}^{4} \Lambda^{-2}$. By taking $\delta<\bar{r}$, we see that $\gamma$ must exit $P\left(x_{0}, r_{0},-r_{0}^{2}\right)$ at some time $\tilde{t}$. First, suppose $\tilde{t}>t_{0}-\Delta t$. Then by the Schwarz 
inequality we get

$$
\begin{aligned}
\int_{\tilde{t}}^{t_{0}} \sqrt{t_{0}-s}\left|\gamma^{\prime}(s)\right|^{2} d s & \geq\left(\int_{\tilde{t}}^{t_{0}}\left|\gamma^{\prime}(s)\right| d s\right)^{2}\left(\int_{\tilde{t}}^{t_{0}}\left(t_{0}-s\right)^{-1 / 2} d s\right)^{-1} \\
& \geq \frac{1}{2} 10^{-2} r_{0}^{2}(\Delta t)^{-1 / 2}>\Lambda
\end{aligned}
$$

where the factor $10^{-2}$ comes from the distance distortion on $P\left(x_{0}, r_{0},-r_{0}^{2}\right)$.

So now we may assume that $\gamma$ exits $P\left(x_{0}, r_{0},-r_{0}^{2}\right)$ at a time $\tilde{t} \leq t_{0}-\Delta t$. Then the conclusion follows immediately from Lemma 4.4.

Proof. Note by Lemma 4.5 and the properness of scalar curvature from [KL17, Theorem 1.3], a minimizing sequence of admissible curves between any two points converges to a smooth minimizing $\mathcal{L}$-geodesic. Now the rest of proof for part (a) is the same as [KL08, Proposition 85.1(a)].

For part (b), suppose that for some $A>0$ the claim is not true. Then there is a sequence of singular Ricci flows $\mathcal{M}_{k}$ which provide a counterexample. In particular, there exists $x_{k} \in B_{t_{0 k}}\left(x_{0 k}, A r_{0 k}\right)$ with $\rho\left(x_{k}\right) \leq r_{k} r_{0 k}$ but at which the $\epsilon$-canonical neighborhood assumption does not hold, where $r_{k} \rightarrow 0$ as $k \rightarrow \infty$.

Omitting the subscripts for a moment, by Lemma 3.5 we can apply a point-picking and find points $\bar{x} \in B_{\bar{t}}\left(x_{0}, 2 A r_{0}\right), \bar{t} \in\left[t_{0}-r_{0}^{2} / 2, t_{0}\right]$ with $\bar{\rho}:=\rho(\bar{x}, \bar{t}) \leq r r_{0}$ satisfying the following: the $\epsilon$-canonical neighborhood assumption does not hold at $(\bar{x}, \bar{t})$, but it holds at all points in $\bar{P}$ with $\rho \leq \bar{\rho} / 2$, where

$$
\bar{P}=\left\{x \in \mathcal{M}_{t}: d_{t}\left(x_{0}(t), x\right) \leq d_{\bar{t}}\left(x_{0}(\bar{t}), \bar{x}\right)+r^{-1} \bar{\rho}, t \in\left[\bar{t}-\frac{1}{4} r^{-2} \bar{\rho}^{2}, \bar{t}\right]\right\} .
$$

The rest of proof is the same as [KL08, Prop 85.1(b)], in which we can extract a convergent subsequence of $\left(\bar{P}_{k}, g_{k}(t), \bar{x}_{k}\right)$ that converges to a $\kappa$-solution. Then this contradicts the assumption of $\bar{x}_{k}$ for large $k$, and thus proves part (b).

Part (c) follows from (b) in the same way as [KL08, Prop 85.1(c)].

The following lemma is a corollary of Proposition 4.1(c). Given the curvature and reduced volume bound at a single point, it provides curvature bound in a backward parabolic neighborhood of this point.

Lemma 4.6. For any $\kappa>0$, there exists $r(\kappa)>0$ such that the following holds: Let $\mathcal{M}$ be a singular Ricci flow, $x_{0} \in \mathcal{M}, \mathfrak{t}\left(x_{0}\right)=t_{0}$. Suppose the reduced volume $\tilde{V}_{x_{0}}(1) \geq \kappa$, and $\mathcal{M}_{t \geq t_{0}-1}$ is 1-positive.

$$
\text { If }|\operatorname{Rm}|\left(x_{0}\right) \leq 1 \text {, then }|\operatorname{Rm}|(x) \leq r^{-2} \text { for all } x \in P\left(x_{0}, r,-r^{2}\right) \text {. }
$$

Proof. We will first show that $|\operatorname{Rm}|(x) \leq r^{-2}$ for all $x \in B_{t_{0}}\left(x_{0}, r\right)$. Then the assertion in the theorem follows immediately from this by [KL08, Theorem 54.2], which gives 
upper bound on curvature at earlier smaller balls, assuming lower bounds for the volume and curvature in a ball.

For any $x \in \mathcal{M}$, put $\bar{\rho}(x)=\sup \left\{r>0:|\operatorname{Rm}|(y) \leq r^{-2}\right.$ for all $\left.y \in B_{\mathfrak{t}(x)}(x, r)\right\}$. Suppose the assertion is not true. Then there is a sequence of singular Ricci flows $\mathcal{M}_{i}$ and points $x_{0 i} \in \mathcal{M}_{i}, \mathfrak{t}\left(x_{0 i}\right)=t_{0 i}$, that satisfy the assumptions, but $\lim _{i \rightarrow \infty} \bar{\rho}\left(x_{0 i}\right)=0$.

By the reduced volume comparison theorem (see e.g. [KL08, Lem 78.11]), $\tilde{V}_{x_{0}}(1) \geq$ $\kappa$ implies that there exists $\kappa^{\prime}(\kappa)>0$ such that $\mathcal{M}_{i}$ is $\kappa^{\prime}$-non-collapsed at $x_{0 i}$ at scales less than 1 . Rescale and do a time-shifting to the flows $\left(\mathcal{M}_{i}, x_{0 i}\right)$ to get a sequence of new flows, which are still denoted by $\left(\mathcal{M}_{i}, x_{0 i}\right)$, such that in the new flows we have $\bar{\rho}\left(x_{0 i}\right)=1$ and $\mathfrak{t}\left(x_{0 i}\right)=0$. So $\operatorname{vol}\left(B_{0}\left(x_{0 i}, 1\right)\right) \geq \kappa^{\prime}>0$ for all $i$.

By [KL08, Theorem 54.2], we can find $C(\kappa)>0$ such that $|\mathrm{Rm}| \leq C$ in $P\left(x_{0 i}, C^{-1 / 2},-C^{-1}\right)$. So by applying Proposition 4.1(c) and some distance distortion estimates, we can find a smooth Ricci flow $\left(U, g_{\infty}(t), x_{\infty}\right)$ (possibly incomplete), $t \in[-c, 0]$, for some $c>0$, such that there are diffeomorphisms $\phi_{i}: U \rightarrow \mathcal{M}_{i}$ such that $\phi_{i}\left(x_{\infty}\right)=x_{0 i}$ and $\lim _{i \rightarrow \infty}\left\|\phi_{i}^{*} g_{i}(t)-g_{\infty}(t)\right\|_{C^{k}(U)}=0$ for all $k \in \mathbb{N}$ and $t \in[-c, 0]$, and $B_{0}\left(x_{\infty}, 2\right)$ is relatively compact in $U$.

By the 1-positive pinching assumption, we see that $\operatorname{Rm}(x, t) \geq 0$ for all $x \in U$ and $t \in[-c, 0]$. Also, we have $|\operatorname{Rm}|\left(x_{\infty}, 0\right)=0, \bar{\rho}\left(x_{\infty}, 0\right)=1$, and hence $|\operatorname{Rm}|(y, 0)=1$ for some $y \in U$. However, this contradicts with the strong maximum principle, see e.g. [MT09, Theorem 4.18].

Theorem 4.2 is a immediate consequence of combining Lemma 4.6 and Proposition 4.1(c).

\section{HEAT KERNEL FOR SINGULAR RICCI FLOW}

Let $\mathcal{M}$ be a singular Ricci flow and $x_{0} \in \mathcal{M}_{t_{0}}, 0<t_{0}<T$. Our first main result in this section is Theorem 5.1, in which we find a positive solution $u$ to the conjugate heat equation $\left(-\partial_{t}-\Delta+R\right) u=0$, which is a $\delta$-function at $x_{0}$. Moreover, $u$ is positive at all points that are accessible to $x_{0}$, and vanishes elsewhere. We also show that outside a backward parabolic neighborhood $P\left(x_{0}, r_{0},-r_{0}^{2}\right)$ around $x_{0}$, we have $u \leq C_{0}$. We say such a function $u$ is the heat kernel of $\mathcal{M}$ starting from $x_{0}$.

The next main result is Theorem 5.5, which improves the bound $u \leq C_{0}$ to the following for any integer $m \geq 1$ :

$$
u R^{m} \leq C_{m}
$$

where $C_{m}>0$ depends on $m$. This indicates that the heat kernel $u$ is sufficiently small at points where the curvature is sufficiently large. With this estimate we are able to show that the heat kernel in Theorem 5.1 has many good properties as those of the ordinary heat kernel for compact smooth Ricci flows.

To compare, let $u(\cdot, \cdot)$ be the heat kernel starting from $\left(x_{0}, t_{0}\right)$ for a smooth compact Ricci flow $(M, g(t)), x_{0} \in M$. Then a direct computation by integration by parts 
shows

$$
\partial_{t} \int u(x, t) d_{t} x=\int\left(\partial_{t} u-R u\right) d_{t} x=\int-\Delta u d_{t} x=0 .
$$

So we get $\int u d_{t} x=1$ for all $t<t_{0}$. For the singular Ricci flow and the generalized heat kernel, we show in Corollary 5.6 that $\int u d_{t} x=1$ also holds for the heat kernel in Theorem 5.1. Also, by a similar argument we show in Corollary 5.7 that Perelman's differential Harnack inequality $v \leq 0$ holds.

\subsection{Construction of the heat kernel.}

Theorem 5.1. Let $\mathcal{M}$ be a singular Ricci flow with normalized initial condition, and $x_{0} \in \mathcal{M}$ with $t_{0}:=\mathfrak{t}\left(x_{0}\right)>0$. Then there exists a function $u: \mathcal{M}_{t<t_{0}} \rightarrow \mathbb{R}$ which is a smooth solution to the conjugate heat equation, i.e. $\left(-\partial_{t}-\Delta+R\right) u=0$, and it satisfies the following properties

(1) $u$ is a positive smooth function on $\mathcal{M}\left(x_{0}\right)$, and $u$ vanishes on $\mathcal{M}-\mathcal{M}\left(x_{0}\right)$. Recall $\mathcal{M}\left(x_{0}\right)$ is the subset of all points that are accessible to $x_{0}$.

(2) $u$ is a delta-function at $x_{0}$, in the sense that $\lim _{t \nearrow t_{0}} \int_{\mathcal{M}_{t}} u(x) h(x) d_{t} x=h\left(x_{0}\right)$ for any smooth function $h$ on $\mathcal{M}$ that has a compact support.

(3) Suppose for some $r_{0}, T>0$ we have $|\mathrm{Rm}| \leq r_{0}^{-2}$ on $\mathcal{P}_{0}:=P\left(x_{0}, r_{0},-r_{0}^{2}\right)$, and $t_{0}<T$. Then there exists $C_{0}\left(r_{0}, T\right)>0$ such that $u \leq C_{0}$ on $\mathcal{M}_{t<t_{0}}-\mathcal{P}_{0}$.

For simplicity, we use the following variant of Perelman's Ricci flow with surgery, in which the surgeries are done slightly before singular times (the times where curvature blows up), instead of exactly at them. This Ricci flow with surgery can be obtained with little modification to that of Perelman's. It is also constructed for complete manifold with bounded geometry in BBM11.

Definition 5.2 (Ricci flow with surgery). A Ricci flow with surgery is given by

(1) A collection of Ricci flows $\left\{\left(M_{k} \times\left[t_{k}^{-}, t_{k}^{+}\right], g_{k}(\cdot)\right)\right\}_{1 \leq k \leq N}$, where $N \leq \infty, M_{k}$ is a compact (possibly empty) manifold, $t_{k}^{+}=t_{k+1}^{-}$for all $1 \leq k \leq N$.

(2) A collection of isometric embeddings $\left\{\psi_{k}: Y_{k}^{+} \rightarrow Y_{k+1}^{-}\right\}_{1 \leq k \leq N}$ where $Y_{k}^{+} \subset M_{k}$ and $Y_{k+1}^{-} \subset M_{k+1}, 1 \leq k<N$, are compact 3 dimensional submanifold with boundary. The $Y_{k}^{ \pm}$'s are the subsets which survive the transition from one flow to the next, and the $\psi_{k}$ 's give the identification between them.

We call each final time $t_{k}^{+}$a surgery time. Let $X_{k}^{+}$and $X_{k+1}^{-}$denote the interior of $Y_{k}^{+}$and $Y_{k+1}^{-}$respectively for $1 \leq k<N$. We can associate a Ricci flow spacetime $\mathcal{M}$ to the Ricci flow with surgery by taking $\mathcal{M}$ to be the disjoint union of

$$
\left(M_{k} \times\left[t_{k}^{-}, t_{k}^{+}\right)\right) \cup\left(X_{k}^{+} \times\left\{t_{k}^{+}\right\}\right)
$$

for $1 \leq k \leq N$, and removing the following subset

$$
\left(M_{k+1}-X_{k+1}^{-}\right) \times\left\{t_{k+1}^{-}\right\}
$$


for all $1 \leq k<N$ (making identifications using the $\psi_{k}$ 's as gluing maps).

Proof of Theorem 5.1. Let $\mathcal{M}_{i}$ be a sequence Ricci flow with surgery spacetimes starting from $(M, g)$, with the surgery scale $\delta_{i} \rightarrow 0$ as $i \rightarrow \infty$. Then $\mathcal{M}_{i}$ have the local control required for the application of the spacetime compactness theorem in KL17, Theorem 2.20], and hence $\mathcal{M}_{i}$ converges to the singular Ricci flow $\mathcal{M}$ as $i \rightarrow \infty$. Assume $x_{0 i} \in \mathcal{M}_{i}$ converges to $x_{0}$ when $i \rightarrow \infty$. We shall construct smooth nonnegative solutions to the conjugate heat equation on each $\mathcal{M}_{i}$ starting from $x_{0 i}$, and then take a limit of them to obtain the desired heat kernel on $\mathcal{M}$.

Let $\mathcal{M}_{i}$ be fixed below, and assume the compact Ricci flows that form $\mathcal{M}_{i}$ are $\left\{\left(M_{k} \times\left[t_{k}^{-}, t_{k}^{+}\right], g_{k}(\cdot)\right)\right\}_{1 \leq k \leq N}$, where $t_{N}^{+}=t_{0 i}=\mathfrak{t}\left(x_{0 i}\right)$. We shall define $u_{i}$ on each of these Ricci flows and then restrict it to $\mathcal{M}_{i}$ to get a smooth function.

First, on $M_{N} \times\left[t_{N}^{-}, t_{N}^{+}\right)$, let $u_{i}$ be the ordinary heat kernel of $\left(M_{N}, g_{N}(t)\right)$ which starts from $\left(x_{0 i}, t_{0 i}\right)$. Note that $u_{i}$ vanishes at $(x, t)$ if $x$ are not in the same component with $x_{0 i}$. Then suppose by induction that $u_{i}$ has been defined on $M_{j} \times\left[t_{j}^{-}, t_{j}^{+}\right)$for all $j=k, \ldots, N$ such that the following holds:

(1) $u_{i} \geq 0$ is a smooth solution to the conjugate heat equation on $\mathcal{M}_{i, t \geq t_{k}^{-}}$.

(2) $u_{i}$ vanishes at points that are not accessible to $x_{0 i} \in \mathcal{M}_{i}$.

(3) $\int_{M_{j}} u_{i}(x, t) d_{t} x \leq 1$ for all $t \in\left[t_{j}^{-}, t_{j}^{+}\right)$, and $j=k, \ldots, N$.

Then we define $u_{i}$ on $M_{k-1} \times\left\{t_{k-1}^{+}\right\}$by letting $u_{i}\left(x, t_{k-1}^{+}\right)=u_{i}\left(x, t_{k}^{-}\right)$if $x \in X_{k-1}^{+}$, and $u_{i}\left(x, t_{k-1}^{+}\right)=0$ if $x \in M_{k-1}-X_{k-1}^{+}$. Then for any $(x, t) \in M_{k-1} \times\left[t_{k-1}^{-}, t_{k-1}^{+}\right)$, set

$$
u_{i}(x, t)=\int_{X_{k-1}^{+}} u_{i}\left(y, t_{k-1}^{+}\right) H\left(y, t_{k-1}^{+} ; x, t\right) d_{t_{k-1}^{+}} y,
$$

where $H(\cdot, \cdot ; \cdot, \cdot)$ is the ordinary heat kernel for the smooth compact Ricci flow $\left(M_{k-1}, g_{k-1}(t)\right)$, $t \in\left[t_{k-1}^{-}, t_{k-1}^{+}\right]$. So $u_{i}$ is a smooth solution to the conjugate heat equation on $M_{k-1} \times\left[t_{k-1}^{-}, t_{k-1}^{+}\right) \cup X_{k-1}^{+} \times\left\{t_{k-1}^{+}\right\}$, and hence it is smooth on $\mathcal{M}_{i, t \geq t_{k-1}^{-}}$. Moreover, by (5.5) we get for all $t \in\left[t_{k-1}^{-}, t_{k-1}^{+}\right)$that

$$
\begin{aligned}
\int_{M_{k-1}} u_{i}(x, t) d_{t} x & \leq \int_{M_{k-1}} \int_{X_{k-1}^{+}} u_{i}\left(y, t_{k-1}^{+}\right) H\left(y, t_{k-1}^{+} ; x, t\right) d_{t_{k-1}^{+}} y d_{t} x \\
& =\int_{X_{k-1}^{+}} u_{i}\left(y, t_{k-1}^{+}\right) d_{t_{k-1}^{+}} y \leq 1 .
\end{aligned}
$$

It is clear that assumptions (1) and (2) also hold for $j=k-1$. So by induction, we obtain a smooth non-negative solution $u_{i}$ on $\mathcal{M}_{i}$ which satisfies $\int_{\mathcal{M}_{i}(t)} u_{i}(x, t) d_{t} x \leq 1$, and $u_{i} \equiv 0$ on $\mathcal{M}_{i}-\mathcal{M}_{i}\left(x_{0 i}\right)$.

Next, suppose $r_{0}, T$ are the constants in assertion (3). Then since $\left(\mathcal{M}_{i}, x_{0 i}\right)$ converges to $\left(\mathcal{M}, x_{0}\right)$, we have $|\mathrm{Rm}| \leq 2 r_{0}^{-2}$ on $\mathcal{P}_{0 i}:=P\left(x_{0 i}, r_{0},-r_{0}^{2}\right)$ for all large $i$. Since $\mathcal{M}_{i}$ has the normalized initial condition, the scalar curvature satisfies $R \geq-6$ 
anywhere on $\mathcal{M}_{i}$, see [KL08, Lemma 79.11]. So $\tilde{u}_{i}:=u_{i} e^{-6\left(t_{0}-t\right)}$ satisfies

$$
\left(-\partial_{t}-\Delta\right) \tilde{u}_{i} \leq 0 \text {. }
$$

Let $\Gamma$ be the parabolic boundary of $\mathcal{P}_{0 i}$, i.e.

$$
\Gamma=\left(\bigcup_{t \in\left[t_{0 i}-r_{0}^{2}, t_{0 i}\right)} \partial B_{t_{0 i}}\left(x_{0 i}, r_{0}\right)(t)\right) \cup B_{t_{0 i}}\left(x_{0 i}, r_{0}\right)\left(t_{0 i}-r_{0}^{2}\right) .
$$

Then since $\int u_{i} d_{t} x \leq 1$, we can apply the parabolic mean value inequality (see e.g. $\left[\mathrm{CCG}^{+} 10\right.$, Theorem 25.2]) at any points in $\Gamma$, and hence find a constant $C_{1}\left(r_{0}\right)>0$ such that $\tilde{u}_{i} \leq C_{1}$ on $\Gamma$.

Suppose by induction that $\tilde{u}_{i} \leq C_{1}$ on $\mathcal{M}_{i,\left[t_{k}^{-}, t_{0}\right)}-\mathcal{P}_{0 i}$ for some $k \leq N$. Without loss of generality, we may assume that $t_{0 i}-r_{0}^{2}$ is a surgery time. Then if $M_{k-1} \times\left[t_{k-1}^{-}, t_{k-1}^{+}\right)$ does not intersect $\mathcal{P}_{0 i}$, by the inductive assumption we can apply the maximum principle and get $\tilde{u}_{i} \leq C_{1}$ on $\mathcal{M}_{i,\left[t_{k-1}^{-}, t_{k-1}^{+}\right)}$. Otherwise, apply the maximum principle on $\bigcup_{t \in\left[t_{k-1}^{-}, t_{k-1}^{+}\right)}\left(M_{k}(t) \backslash B_{t_{0 i}}\left(x_{0 i}, r_{0}\right)(t)\right)$, whose boundary is contained in $\Gamma$. Then by inductive assumption and $\tilde{u}_{i} \leq C_{1}$ on $\Gamma$, we get $\tilde{u}_{i} \leq C_{1}$ on $\mathcal{M}_{i,\left[t_{k-1}^{-}, t_{k-1}^{+}\right)}$. So by induction, $\tilde{u}_{i} \leq C_{1}$ holds on $\mathcal{M}_{i, t<t_{0 i}}-\mathcal{P}_{0 i}$. Let $C_{0}=C_{1} e^{6 T}$, then $u_{i} \leq C_{0}$ on $\mathcal{M}_{i, t<t_{0 i}}-\mathcal{P}_{0 i}$.

Then applying the interior Hölder estimate, we can bound the derivatives of $u_{i}$ in terms of its $C^{0}$-norm and the curvature norm nearby. So by passing to a subsequence, we may assume that $u_{i}$ converges smoothly to a non-negative smooth solution $u$ to the conjugate heat equation on $\mathcal{M}$. It follows immediately that

$$
u(x) \leq C_{0}
$$

for all $x \in \mathcal{M}-\mathcal{P}_{0}$, which proves assertion (3) in the theorem.

Now we establish the properties claimed in Theorem 5.1. First, we show that $u$ is a $\delta$-function at $x_{0}$. For large $i$, let $\phi_{i}$ be a cut-off function whose support is contained in $B_{t_{0 i}}\left(x_{0 i}, r_{0}\right), \phi_{i} \equiv 1$ on $B_{t_{0 i}}\left(x_{0 i}, \frac{r_{0}}{2}\right)$, and $\left|\nabla \phi_{i}\right|$ and $\left|\Delta \phi_{i}\right|$ are bounded above in terms of $r_{0}$. Here the derivatives and norms are considered with respect to $g\left(t_{0 i}\right)$. By the choice of $r_{0}$, there exists a universal constant $c>0$ such that $\frac{1}{2} g(t) \leq g\left(t_{0 i}\right) \leq 2 g(t)$ for all $t \in\left[t_{0 i}-c r_{0}^{2}, t_{0 i}\right]$ on $B_{t_{0 i}}\left(x_{0 i}, r_{0}\right)$. So for all $t \in\left[t_{0 i}-c r_{0}^{2}, t_{0 i}\right)$, a direct computation using integration by parts shows that there is a constant $C=C\left(r_{0}\right)>0$ such that

$$
\partial_{t} \int_{B_{t_{0 i}}\left(x_{0 i}, r_{0}\right)} u_{i} \phi_{i} d_{t} x=\int_{B_{t_{0 i}}\left(x_{0 i}, r_{0}\right)}-u_{i} \Delta_{g(t)} \phi_{i} d_{t} x \leq\left|\Delta_{g(t)} \phi_{i}\right| \leq C,
$$

where we also used $\int_{\mathcal{M}_{i, t}} u_{i}(x, t) d_{t} x \leq 1$. Integrating this we get for all $t \in\left[t_{0 i}-\right.$ $\left.c r_{0}^{2}, t_{0 i}\right)$ that $\int_{B_{t_{0 i}}\left(x_{0 i}, r_{0}\right)} u_{i}(x, t) d_{t} x \geq 1-C\left(t_{0 i}-t\right)$. Passing to the limit this implies

$$
\int_{\left(B_{t_{0}}\left(x_{0}, r_{0}\right)\right)(t)} u(x) d_{t} x \geq 1-C\left(t_{0}-t\right) .
$$


Letting $t \nearrow t_{0}$, we get

$$
\lim _{t \nearrow t_{0}} \int_{\mathcal{M}_{t}} u(x) d_{t} x=1
$$

By a same argument we can show that $\lim _{t} t_{0} \int_{\mathcal{M}_{t}} u(x) h(x) d_{t} x=h\left(x_{0}\right)$, for all smooth function $h$ that has compact support. So $u$ is a $\delta$-function at $x_{0}$, which verifies assertion (2) in Theorem 5.1,

Now we verify assertion (1) in Theorem 5.1, First, the positivity of $u$ on $\mathcal{M}\left(x_{0}\right)$ is an easy consequence of the Harnack inequality for parabolic equations. So it remains to show that $u$ vanishes on $\mathcal{M}-\mathcal{M}\left(x_{0}\right)$.

To show this, let $y \in \mathcal{M}-\mathcal{M}\left(x_{0}\right), \mathfrak{t}(y)=t \in\left[0, t_{0}\right)$. Since by [KL18, Theorem 6.3] the non-singular times, at which the time-slices have bounded curvature, are dense, we may assume without loss of generality that $t$ is a non-singular time. Otherwise, we can find a sequence of non-singular times $s_{k}>t$ that converges to $t$ as $k \rightarrow \infty$, and $y\left(s_{k}\right) \in \mathcal{M}-\mathcal{M}\left(x_{0}\right)$. So $\mathcal{M}_{t}$ has finitely many connected components which are closed manifolds. Since $y \in \mathcal{M}-\mathcal{M}\left(x_{0}\right)$, it is easy to see that $x_{0}(t)$ and $y$ are in different connected components in $\mathcal{M}_{t}$.

Then by [KL17, Theorem 1.3], there is a sequence of time-preserving diffeomorphisms $\left\{\phi_{i}: U_{i} \rightarrow \mathcal{M}_{i}\right\}$, where $U_{i}$ are open subsets of $\mathcal{M}$ such that given any $\bar{t}, \bar{R}>0$, if $i$ is sufficiently large then

$$
U_{i} \supset\{x \in \mathcal{M}: \mathfrak{t}(x) \leq \bar{t} \text { and } R(x) \leq \bar{R}\},
$$

and $\left\{\phi_{i}^{*} g_{i}\right\}$ converges smoothly on compact subsets of $\mathcal{M}$ to $g$. So $\mathcal{M}_{t}$ is contained in $U_{i}$ for all large $i$ and $\phi_{i}\left(\mathcal{M}_{t}\right)$ is a finite union of closed manifolds. In particular, $\phi_{i}\left(x_{0}(t)\right)$ and $\phi_{i}(y)$ are in different connected components in $\phi_{i}\left(\mathcal{M}_{t}\right)$, which implies $\phi_{i}(y) \in \mathcal{M}_{i}-\mathcal{M}\left(x_{0 i}\right)$. So $u_{i}$ vanishes at $\phi_{i}(y)$. Letting $i \rightarrow \infty$ we get $u(y)=0$.

\subsection{Further properties of the heat kernel.}

In this subsection we investigate more properties of the heat kernel in Theorem 5.1. The first main result is Theorem 5.4, which is a semi-local maximum principle for the heat kernel. Then in Theorem 5.5 we derive from Theorem 5.4 a polynomial decay estimate of the heat kernel. Corollary 5.6 and 5.7 are applications of Theorem 5.5.

The main ingredient in the proof of the semi-local maximum principle is the following vanishing theorem of the Bryant soliton:

Proposition 5.3. (Vanishing theorem on Bryant soliton) Let $(M, g(t)), t \in \mathbb{R}$ be a Bryant soliton with tip $x_{0} \in M, R\left(x_{0}, 0\right)=1$, and $u(x, t): M \times[0, \infty) \rightarrow \mathbb{R}$ be a smooth non-negative solution to the conjugate heat equation. Suppose there are constants $C>0$ and $m \in \mathbb{N}_{+}$such that $u(x, t) R^{m}(x, t) \leq C$ for all $x \in M$ and $t \in[0, \infty)$.

\section{Then $u \equiv 0$.}


Proof. Let $C$ denote all the constants that depend only on the constants $C$ and $m$ in the assumption. Without loss of generality, it suffices to show $u(x, 0)=0$ for all $x \in M$.

Let $H(y, t ; z, s), t>s$ be the heat kernel of $(M, g(t))$. Then the following holds for all $t>0$

$$
\begin{aligned}
u(x, 0) & =\int H(y, t ; x, 0) u(y, t) d_{t} y \\
& =\int_{d_{t}\left(y, x_{0}\right) \leq 1} H(y, t ; x, 0) u(y, t) d_{t} y+\int_{d_{t}\left(y, x_{0}\right)>1} H(y, t ; x, 0) u(y, t) d_{t} y \\
& =\mathcal{I}(t)+\mathcal{J}(t) .
\end{aligned}
$$

We shall show that $\mathcal{I}(t)$ and $\mathcal{J}(t)$ converge to 0 as $t \rightarrow \infty$. First, to estimate $\mathcal{I}(t)$ we note that $d_{t}\left(y, x_{0}\right) \leq 1$ implies $R(y, t)>C^{-1}$. So it follows from the assumption $u R^{m} \leq C$ and the Bishop-Gromov volume comparison that

$$
\mathcal{I}(t) \leq C \int_{d_{t}\left(y, x_{0}\right) \leq 1} H(y, t ; x, 0) d_{t} y \leq C \sup _{d_{t}\left(y, x_{0}\right) \leq 1} H(y, t ; x, 0) .
$$

By the Gaussian bound for heat kernel of a Ricci flow with bounded curvature $\left[\mathrm{CCG}^{+} 10\right.$, Corollary 26.26], we have $\lim _{t \rightarrow \infty} H(y, t ; x, 0)=0$ uniformly for all $y \in M$. So $\mathcal{I}(t) \rightarrow 0$, as $t \rightarrow \infty$.

Next we estimate $\mathcal{J}(t)$. Since $d_{t}\left(y, x_{0}\right) \geq 1$, it follows that $C^{-1} d_{t}\left(y, x_{0}\right)^{-1} \leq$ $R(y, t) \leq C d_{t}\left(y, x_{0}\right)^{-1}$, which together with $u R^{m} \leq C$ implies

$$
\mathcal{J}(t) \leq C \int_{d_{t}\left(y, x_{0}\right)>1} H(y, t ; x, 0) d_{t}^{m}\left(y, x_{0}\right) d_{t} y .
$$

We claim that the following estimate holds for all $(y, t)$ such that $t \geq C$ and $d_{t}\left(y, x_{0}\right) \geq$ 1:

$$
H(y, t ; x, 0) \leq C \exp \left(-\frac{d_{0}^{2}(x, y)}{C t}\right)
$$

Assume for a moment that the claim is true, and we use it to prove the proposition. For any $(y, t)$ such that $t \geq C$ and $d_{t}\left(y, x_{0}\right) \geq 1$, we have $d_{s}\left(y, x_{0}\right) \geq 1$ for all $s \in[0, t]$ because of Ric $\geq 0$. Since $R \geq C^{-1}$ on $B_{s}\left(x_{0}, 1\right)$ for any $s \in \mathbb{R}$, by a distance distortion estimate we get $d_{s}\left(x_{0}, y\right) \geq C^{-1}(t-s)$ for all $s \in[0, t]$. So when $t \geq C$ we have

$$
d_{0}(x, y) \geq d_{0}\left(x_{0}, y\right)-d_{0}\left(x, x_{0}\right) \geq d_{0}\left(x_{0}, y\right)\left(1-\frac{d_{0}\left(x, x_{0}\right)}{C^{-1} t}\right) \geq \frac{1}{2} d_{0}\left(x_{0}, y\right)
$$

substituting which into (5.17) we get

$$
H(y, t ; x, 0) \leq C \exp \left(-\frac{d_{0}^{2}\left(x_{0}, y\right)}{C t}\right) .
$$


Putting this into (5.16) and using the Bishop-Gromov volume comparison theorem we get

$$
\mathcal{J}(t) \leq \int_{d_{0}\left(y, x_{0}\right)>C^{-1} t} C \exp \left(-\frac{d_{0}^{2}\left(x_{0}, y\right)}{C t}\right) d_{0}^{m}\left(x_{0}, y\right) d_{0} y \leq C e^{-\frac{t}{C}} .
$$

Hence $\mathcal{J}(t) \rightarrow 0$ as $t \rightarrow \infty$. Therefore, by letting $t \rightarrow \infty$, we obtain $u(x, 0)=0$.

Now we establish (5.17) to finish the proof. Fix a pair $(y, t)$ such that $t \geq C$ and $d_{t}\left(y, x_{0}\right) \geq 1$. The value of $C$ will be determined later. For any $s \in[0,1]$ and $z \in B_{s}(y, 1)$, let $\ell(z, s)$ be the reduced length from $(z, s)$ to $(y, t)$, and let $\gamma:[s, t] \rightarrow M$ be a curve such that $\left.\gamma\right|_{[2, t]} \equiv y$ and $\left.\gamma\right|_{[s, 2]}$ is a minimal geodesic connecting $y$ and $z$ with respect to $g(0)$.

For $\tau \in[0, t-s]$, we have $R(y, t-\tau) \leq C d_{t-\tau}^{-1}\left(y, x_{0}\right)$ and $d_{t-\tau}\left(y, x_{0}\right) \geq C^{-1} \tau$, and hence $R(y, t-\tau) \leq \frac{C}{\tau}$. For $\tau \in[t-2, t-s]$, we have $R(\gamma(t-\tau), t-\tau) \leq C$ and $\left|\gamma^{\prime}\right|(t-\tau) \leq C$. Putting these together we can estimate the $\mathcal{L}$-length of $\gamma$ :

$$
\begin{aligned}
\mathcal{L}(\gamma) & =\int_{0}^{t-2} \sqrt{\tau} R(y, \tau) d \tau+\int_{t-2}^{t-s} \sqrt{\tau}\left(R(\gamma(t-\tau), t-\tau)+\left|\gamma^{\prime}\right|^{2}\right) d \tau \\
& \leq \int_{0}^{t-2} \sqrt{\tau} \frac{C}{\tau} d \tau+\int_{t-2}^{t-s} C \sqrt{\tau} d \tau \leq C \sqrt{t}
\end{aligned}
$$

and hence

$$
\ell(z, s)=\frac{\mathcal{L}(z, s)}{2 \sqrt{t-s}} \leq \frac{\mathcal{L}(\gamma)}{2 \sqrt{t-s}} \leq C .
$$

Recall the heat kernel lower bound by Perelman in [Per02, Corollary 9.5] we get:

$$
H(y, t ; z, s) \geq \frac{1}{4 \pi(t-s)^{3 / 2}} e^{-\ell(z, s)} \geq \frac{C}{t^{\frac{3}{2}}},
$$

for all $s \in[0,1]$ and $z \in B_{s}(y, 1)$. So by the Bishop-Gromov volume comparison we get

$$
\int_{B_{s}(y, 1)} H(y, t ; z, s) d_{s} z \geq \frac{C}{t^{3 / 2}} \geq \frac{C}{d_{0}(x, y)^{3 / 2}}
$$

for all $s \in[0,1]$.

Note by the multiplication inequality for heat kernel in HN14 we have

$$
\left(\int_{B_{s}(y, 1)} H(y, t ; z, s) d_{s} z\right)\left(\int_{B_{s}(x, 1)} H(y, t ; z, s) d_{s} z\right) \leq C \exp \left(-\frac{\left(d_{s}(x, y)-2\right)^{2}}{4 C(t-s)}\right) .
$$

So by substituting (5.24) into (5.25) we get

$$
\left(\int_{B_{s}(x, 1)} H(y, t ; z, s) d_{s} z\right) \leq C d_{0}(x, y)^{\frac{3}{2}} \exp \left(-\frac{d_{0}(x, y)^{2}}{4 C(t-s)}\right) \leq C \exp \left(-\frac{d_{0}(x, y)^{2}}{4 C(t-s)}\right),
$$


where we also used $d_{s}(x, y)-2 \geq C^{-1} d_{0}(x, y)-2 \geq C^{-1} d_{0}(x, y)$ for $t$ very large. Integrating this for all $s \in[0,1]$, and then applying the parabolic mean value inequality (see e.g. [CCG $\left.{ }^{+} 10\right)$ to $H(y, t ; \cdot, \cdot)$ at $(x, 0)$, we obtain

$$
H(y, t ; x, 0) \leq C \exp \left(-\frac{d_{0}^{2}(x, y)}{4 C t}\right),
$$

which confirms claim (5.17) and hence completes the proof.

Proposition 5.4. (A semi-local Maximum Principle) Given $r_{0}, T>0, r_{0}^{2}<T$ and $m \in \mathbb{N}$, there exist $\epsilon=\epsilon\left(r_{0}, T, m\right)>0$ and $r=r\left(r_{0}, T, m\right)>0$ such that for any $t_{0} \in\left(r_{0}^{2}, T\right)$ the following holds:

Let $\mathcal{M}$ be a singular Ricci flow with normalized initial condition, $x_{0} \in \mathcal{M}, \mathfrak{t}\left(x_{0}\right)=$ $t_{0}>0$. Suppose $|\mathrm{Rm}| \leq r_{0}^{-2}$ on $\mathcal{P}_{0}:=P\left(x_{0}, r_{0},-r_{0}^{2}\right)$. Let $u$ be the heat kernel in Theorem 5.1 which starts from $x_{0}$. Then for any $x \in \mathcal{M}_{t<t_{0}}-\mathcal{P}_{0}$ with $R(x)>r^{-2}$, there exists $y \in \mathcal{M}_{t<t_{0}}-\mathcal{P}_{0}$ with $\mathfrak{t}(y) \geq \mathfrak{t}(x)$ such that

$$
\left\{\begin{array}{l}
u(y) \geq(1+\epsilon) u(x) \quad \text { and } \\
\left(u R^{m}\right)(y) \geq(1+\epsilon)\left(u R^{m}\right)(x) .
\end{array}\right.
$$

Proof. Suppose the conclusion is not true, then there are sequences $\left\{\epsilon_{k}\right\}$ and $\left\{r_{k}\right\}$ both converging to zero, and a sequence of Ricci flow spacetime $\left(\mathcal{M}_{k}, g_{k}(t)\right)$, along with the heat kernels $u_{k}$ starting from $x_{0 k} \in \mathcal{M}_{k}, t_{0 k}=\mathfrak{t}\left(x_{0 k}\right)$, which contradict the lemma at points $x_{k} \in \mathcal{M}_{k, t<t_{0 k}}-\mathcal{P}_{0 k}, \mathfrak{t}\left(x_{k}\right)=t_{k}$. This means $\rho\left(x_{k}\right)<r_{k}$, and for any $y \in \mathcal{M}_{k, t<t_{0 k}}-\mathcal{P}_{0 k}$ with $\mathfrak{t}(y) \geq t_{k}$, we have either

$$
u_{k}(y)<\left(1+\epsilon_{k}\right) u_{k}\left(x_{k}\right)
$$

or

$$
\left(u_{k} R^{m}\right)(y)<\left(1+\epsilon_{k}\right)\left(u_{k} R^{m}\right)\left(x_{k}\right) .
$$

This implies $u\left(x_{k}\right)>0$, and by item (1) in Theorem 5.1 we get $x_{k} \in \mathcal{M}_{k}\left(x_{0 k}\right)$.

Let $\rho_{0}=\min \left\{\frac{1}{2} r_{0}, r\left(\epsilon_{\text {can }}, T+1\right)\right\}$, where $r(\cdot, \cdot)$ is the canonical neighborhood scale function for singular Ricci flow with normalized initial condition in Definition 2.19, and $\epsilon_{\text {can }}$ is sufficiently small. Let $P_{k}=P\left(x_{k}, \frac{\rho_{0}}{4 \eta}, \frac{\rho_{0}^{2}}{4 \eta}\right) \subset \mathcal{M}_{k}$, where $\eta>0$ is from Lemma 2.15. Then by Lemma 2.15 we have $\rho<r_{0}$ on $P_{k}$ for all large $k$. Seeing that $\rho \geq r_{0}$ on $\mathcal{P}_{0 k}$, it implies that $P_{k} \subset \mathcal{M}_{k, t<t_{0 k}}-\mathcal{P}_{0 k}$. Now rescaling the spacetimes in $P_{k}$ by $R\left(x_{k}\right)$ and shifting the times $t_{k}$ to 0 , we get a sequence of Ricci flow spacetimes $\left(\tilde{P}_{k}, \tilde{g}_{k}(s)_{s \geq 0},\left(x_{k}, 0\right)\right)$, where $\tilde{g}_{k}(s)$ denotes the horizontal Riemannian metric. Since $r_{k} \rightarrow 0$ as $k \rightarrow \infty$, by Lemma 3.5 we may assume by passing to a subsequence that $\left(\tilde{P}_{k}, \tilde{g}_{k}(s)_{s \geq 0},\left(x_{k}, 0\right)\right)$ converges to a $\kappa$-solution $\left(M_{\infty}, g_{\infty}(s)_{s \geq 0},\left(x_{\infty}, 0\right)\right)$.

Let $\tilde{u}_{k}(y)=\frac{u_{k}(y)}{u_{k}\left(x_{k}\right)}$ for all $y \in \tilde{P}_{k}$. Then for all $y \in \tilde{P}_{k}$ we have either

$$
\tilde{u}_{k}(y)<1+\epsilon_{k},
$$

or

$$
\left(\tilde{u}_{k} R^{m}\right)(y)<1+\epsilon_{k} .
$$


Then since $R>0$ on $\left(M_{\infty}, g_{\infty}(s), x_{\infty}\right)$, we deduce that $\tilde{u}_{k}$ has locally bounded $C^{0}$ norm. By Hölder estimate this implies that the $C^{k}$-norm of $\tilde{u}_{k}$ is locally bounded bound for any $k \in \mathbb{N}$. So by passing to a subsequence we may assume that $\tilde{u}_{k}$ converges smoothly to a smooth non-negative solution $\tilde{u}$ to the conjugate heat equation of the flow $\left(M_{\infty}, g_{\infty}(s)_{s \geq 0}, x_{\infty}\right)$, and $\tilde{u}\left(x_{\infty}, 0\right)=1$. Since $\epsilon_{k} \rightarrow 0$ as $k \rightarrow \infty$, we have that one of the following holds for all $y \in M_{\infty}$ and $s \geq 0$,

$$
\tilde{u}(y, s) \leq 1
$$

or

$$
\left(\tilde{u} R^{m}\right)(y, s) \leq 1
$$

We claim that $\left(M_{\infty}, g_{\infty}(s)\right)$ is either a cylindrical solution (the standard solution on $S^{2} \times \mathbb{R}$, or its quotient by the map that is a reflection on $\mathbb{R}$ and an antipodal map on $S^{2}$ ), or the Bryant soliton. Using the classification result of non-compact $\kappa$-solutions [Bre18], it suffices to show that $M_{\infty}$ is not compact.

Suppose this is not true. On the one hand, by the compactness of $M_{\infty}$, for large $k$ there exists a diffeomorphism $\phi_{k}: M_{\infty} \rightarrow U_{k}$ such that $\phi_{k}\left(x_{\infty}\right)=x_{k}$, where $U_{k}=\phi_{k}\left(M_{\infty}\right)$ is a connected component in $\mathcal{M}_{k, t_{k}}$ and $x_{k} \in U_{k}$. Also, for any given $\delta>0$, the following holds:

$$
\left\|r_{k}^{-2} \phi_{k}^{*}\left(g_{k}\left(t_{k}\right)\right)-g_{\infty}(0)\right\|_{C^{\left[\delta^{-1}\right]}\left(M_{\infty}, g_{\infty}(0)\right)} \leq \delta
$$

On the other hand, since $u_{k}\left(x_{k}\right)>0$ and by item 1 in Theorem 5.1 we see that $x_{k} \in \mathcal{M}_{k}\left(x_{0 k}\right)$. By the component stability theorem, [KL17, Proposition 5.32], for any $t<t_{0 k}$, the time-t-slice of $\mathcal{M}_{k}\left(x_{0 k}\right)$ is the connected component of $\mathcal{M}_{k, t}$ that contains $x_{0 k}(t)$. So we deduce that $U_{k}$ is equal to $\mathcal{M}_{k}\left(x_{0 k}\right)\left(t_{k}\right)$, which is the time- $t_{k}$-slice of $\mathcal{M}_{k}\left(x_{0 k}\right)$.

Since $\inf _{M_{\infty}} R(\cdot, 0) \geq c$ for some $c>0$, by (5.35) we get

$$
\inf _{\mathcal{M}_{k}\left(x_{0 k}\right)\left(t_{k}\right)} R \geq \frac{1}{2} c r_{k}^{-2}
$$

for all large $k$. Then by the maximum principle for scalar curvature we get

$$
R\left(x_{0 k}\right) \geq \inf _{\mathcal{M}_{k}\left(x_{0 k}\right)\left(t \geq t_{k}\right)} R \geq \frac{1}{2} c r_{k}^{-2} .
$$

For sufficiently large $k$, this contradicts the assumption $R\left(x_{0 k}\right) \leq r_{0}^{-2}$. So $M_{\infty}$ must be non-compact.

So first we suppose $\left(M_{\infty}, g_{\infty}(s)\right), s \in[0, \infty)$ is the Bryant soliton. Since the curvature is uniformly bounded everywhere, if $\tilde{u}(y, s) \leq 1$ for some $(y, s) \in M_{\infty} \times[0, \infty)$, then

$$
\tilde{u}(y, s) R^{m}(y, s) \leq C,
$$

where $C$ depends only on the curvature at the tip of $\left(\mathcal{M}_{\infty}, g_{\infty}(0)\right)$. Combining with (5.34), we see that (5.38) holds at all $(y, s) \in M_{\infty} \times[0, \infty)$. By the vanishing theorem, Proposition 5.3, we get $\tilde{u}\left(x_{\infty}, 0\right)=0$, contradiction. 
Next, suppose $\left(M_{\infty}, g_{\infty}(s)\right)$, is a cylindrical solution with $R\left(x_{\infty}, 0\right)=1$. Then the flow exists on $\left[0, \frac{3}{2}\right)$, and $R(y, s) \geq 1$ for all $y \in M_{\infty}$ and $s \in\left[0, \frac{3}{2}\right)$. So (5.34) implies (5.33), and hence $\tilde{u}(y, s) \leq 1$ for all $(y, s) \in M_{\infty} \times\left[0, \frac{3}{2}\right)$. Noting $\tilde{u}\left(x_{\infty}, 0\right)=1$, we can apply the maximum principle at $\left(x_{\infty}, 0\right)$ and get

$$
\left(-\partial_{t}-\Delta\right) \tilde{u} \geq 0, \quad \text { at } \quad\left(x_{\infty}, 0\right) .
$$

This is impossible seeing that $\left(-\partial_{t}-\Delta+R\right) \tilde{u}=0$ and $\tilde{u}\left(x_{\infty}, 0\right) R\left(x_{\infty}, 0\right)>0$.

Theorem 5.5. Under the same assumption as in Proposition 5.4, there exists $C_{m}=$ $C\left(r_{0}, T, m\right)>0$ such that the following holds for all $x \in \mathcal{M}_{t<t_{0}}-\mathcal{P}_{0}$ :

$$
u(x) R^{m}(x) \leq C_{m}
$$

Proof. Let $C_{m}=C_{0} r^{-2 m}$, where $C_{0}=C_{0}\left(r_{0}, T\right)$ is from item (3) in Theorem 5.1, and $r=r\left(r_{0}, T, m\right)>0$ is from Theorem 5.4. Then it's clear that $u R^{m} \leq C_{m}$ for all the points in $\mathcal{M}_{t<t_{0}}-\mathcal{P}_{0}$ that satisfy $R \leq r^{-2}$. We shall show that $u R^{m} \leq C_{m}$ holds everywhere on $\mathcal{M}_{t<t_{0}}-\mathcal{P}_{0}$. Suppose by contradiction that this is not true. Then there exists $x_{1} \in \mathcal{M}_{t<t_{0}}-\mathcal{P}_{0}$ such that $u R^{m}\left(x_{1}\right)>C_{m}$ and $R\left(x_{1}\right)>r^{-2}$.

Suppose by induction that there are $\left\{x_{k}\right\} \subset \mathcal{M}_{t<t_{0}}-\mathcal{P}_{0}, t_{k}=\mathfrak{t}\left(x_{k}\right), k=1,2, \ldots, N$, such that $t_{k} \geq t_{k-1}$, and the following holds for all $k$ :

$$
\left\{\begin{array}{l}
u R^{m}\left(x_{k}\right) \geq(1+\epsilon) u R^{m}\left(x_{k-1}\right), \quad \text { and } \\
u\left(x_{k}\right) \geq(1+\epsilon) u\left(x_{k-1}\right),
\end{array}\right.
$$

where $\epsilon=\epsilon\left(r_{0}, T, m\right)>0$ is from Theorem 5.4. Since $u R^{m}\left(x_{N}\right) \geq u R^{m}\left(x_{1}\right)>C_{m}$, it follows from the definition of $C_{m}$ that $R\left(x_{N}\right)>r^{-2}$. This allows us to apply Proposition 5.4 and get a point $x_{N+1} \in \mathcal{M}_{t<t_{0}}-\mathcal{P}_{0}, \mathfrak{t}\left(x_{N+1}\right)=t_{N+1} \geq t_{N}$ which together with $x_{N}$ satisfies (5.41). So by induction we get an infinite sequence $\left\{x_{k}\right\}_{k=1}^{\infty}$ which satisfies (5.41). Then we can deduce from the second inequality in (5.41) that $u\left(x_{k}\right) \rightarrow \infty$ as $k \rightarrow \infty$, which contradicts item (3) in Theorem 5.1.

Corollary 5.6. Under the same assumption as in Proposition 5.4. Then

$$
\int_{\mathcal{M}_{t}} u d_{t} x=1
$$

for all $t \in\left[0, t_{0}\right)$.

Proof. Without loss of generality, we may assume $t=0$. First, we fix some small $\delta_{\#}>0$ and $\epsilon<\epsilon_{c a n}\left(\delta_{\#}\right)$ from Lemma 2.16. Let $\eta$ be from Lemma 2.15, Let $m \in \mathbb{N}$ be greater than 1 . We use $C$ to denote all the constants depending on $\delta_{\#}, r_{0}, T$ and $m$.

Let $\delta>0$, whose value will be determined in the course of the proof. Choose a division of $\left[0, t_{0}\right]$ by $0=t_{1}<t_{2}<\ldots<t_{N}=t_{0}$, such that $t_{i+1}-t_{i} \leq \delta^{2}$ for all $i=1, \ldots, N-1$, and $N \leq\left(t_{0}+1\right) \delta^{-2} \leq(T+1) \delta^{-2}$. By Lemma [2.18, for sufficiently small $\delta$, there is an open domain $\Omega \subset \mathcal{M}_{t_{2 i+1}}$ such that the boundary components of $\Omega$ are finitely many central spheres in some $\delta_{\#}$-neck, whose number is bounded by $C$, 
and the area of $\partial \Omega$ is less than $C \delta^{2}$. Moreover, we have $\rho \geq 2 \sqrt{\eta} \delta$ on $\Omega$, and $\rho \leq C \delta$ on $\mathcal{M}_{t_{2 i+1}}-\Omega$.

Then by Lemma 2.15 we see that $\sqrt{\eta} \delta \leq \rho(x(t)) \leq C \delta$ for all $x \in \Omega$ and $t \in$ $\left[t_{2 i}, t_{2 i+1}\right]$. So $\Omega$ survives until time $t_{2 i}$, and Area $_{t}(\partial \Omega(t)) \leq C \delta^{2}$. Then applying Theorem 5.5 at points in $\partial \Omega(t)$ and then using the interior Hölder estimate, we get $|\nabla u| \leq C \delta^{2 m-1}$ on $\partial \Omega(t)$. Let $t \in\left[t_{2 i}, t_{2 i+1}\right]$, then

$$
\partial_{t} \int_{\Omega(t)} u(x) d_{t} x=\int_{\Omega(t)}-\Delta u(x) d_{t} x=\int_{\partial \Omega(t)} \frac{\partial u}{\partial \vec{n}} d_{t} S \leq C \cdot \delta^{2 m-2},
$$

where $\vec{n}$ is the inwards unit normal vector field on $\partial \Omega(t)$. Integrating this on $\left[t_{2 i}, t_{2 i+1}\right]$, we get

$$
\int_{\Omega\left(t_{2 i}\right)} u(x) d_{t_{2 i}} x \geq \int_{\Omega} u(x) d_{t_{2 i+1}} x-C \delta^{2 m} .
$$

Also, applying Theorem 5.5 on $\mathcal{M}_{t_{2 i+1}}-\Omega$ and using $\operatorname{vol}\left(\mathcal{M}_{t_{2 i+1}}\right) \leq C$, we get

$$
\int_{\mathcal{M}_{t_{2 i+1}} \Omega} u(x) d_{t_{2 i+1}} x \leq C \delta^{2 m}
$$

which combining with (5.44) gives

$$
\int_{\mathcal{M}_{t_{2 i}}} u(x) d_{t_{2 i}} x \geq \int_{\mathcal{M}_{t_{2 i+1}}} u(x) d_{t_{2 i+1}} x-C \delta^{2 m} .
$$

Note $\lim _{t \nearrow t_{0}} u(x) d_{t} x=1$, by induction we have

$$
\int_{\mathcal{M}_{0}} u(x) d_{0} x \geq 1-C N \delta^{2 m} \geq 1-C(T+1) \delta^{2 m-2} .
$$

Letting $\delta \rightarrow 0$, the conclusion follows immediately.

Corollary 5.7. Under the same assumption as in Proposition 5.4. Let $f$ be a smooth function on $\mathcal{M}\left(x_{0}\right)$ such that $u=\left(4 \pi\left(t_{0}-t\right)\right)^{-3 / 2} e^{-f}$. Then

$$
v=\left[\left(t_{0}-t\right)\left(2 \Delta f-|\nabla f|^{2}+R\right)+f-n\right] u \leq 0 .
$$

Proof. Suppose the conclusion does not hold. Then without loss of generality we may assume that there exists $x_{1} \in \mathcal{M}_{0}$ such that $v\left(x_{1}\right)>0$. Let $h_{0} \geq 0$ be a smooth function on $\mathcal{M}_{0}$ which is supported in a neighborhood of $x_{1}$ in which $v>0$, and $h_{0}\left(x_{1}\right)>0$. Then $\int_{\mathcal{M}_{0}} h_{0} v d_{0} x>0$. In the same way we constructed $u$, we can find a smooth and bounded function $h \geq 0$ on $\mathcal{M}$ with $h(x)=h_{0}(x)$ for all $x \in \mathcal{M}_{0}$, which solves the heat equation $\left(\partial_{t}-\Delta\right) h=0$.

Since $\left(-\partial_{t}-\Delta+R\right) v \leq 0$, see e.g. [KL08], for any open domain $\Omega \subset \mathcal{M}_{t}$ with smooth boundary, we have

$$
\partial_{t} \int_{\Omega}-h v d_{t} V \leq \int_{\partial \Omega}\left(\frac{\partial h}{\partial \vec{n}} v-\frac{\partial v}{\partial \vec{n}} h\right) d_{t} S .
$$


Applying Theorem 5.5 in the same way as Corollary [5.6, we get

$$
\int_{\mathcal{M}_{t}} h v d_{t} x \leq \lim _{s \nearrow t_{0}} \int_{\mathcal{M}_{s}} h v d_{s} x
$$

for all $t \in\left[0, t_{0}\right)$. It was shown in [Ni06] that $\int_{\mathcal{M}_{t}} h v d_{t} x$ approaches to zero as $t$ goes up to $t_{0}$. So (5.50) implies $\int_{\mathcal{M}_{0}} h v d_{0} x \leq 0$, a contradiction.

\section{Pseudolocality theorem on singular Ricci flow}

In this section, we generalize Perelman's pseudolocality theorem for compact Ricci flows to singular Ricci flows. The main ingredient is the heat kernel in Section 5, especially Corollary 5.6 and 5.7.

Theorem 6.1. (Pseudolocality theorem) For every $\alpha>0$ there exists $\delta, \epsilon>0$ with the following property. Let $(\mathcal{M}, g(t))$ be a singular Ricci flow and $x_{0} \in \mathcal{M}_{t_{0}}$ for some $t_{0} \geq 0$. Suppose $R \geq-1$ on $B_{t_{0}}\left(x_{0}, 2\right)$, and for any $\Omega \subset B_{t_{0}}\left(x_{0}, 2\right)$ we have $\operatorname{vol}(\partial \Omega)^{3} \geq(1-\delta) c_{3} \operatorname{vol}(\Omega)^{2}$, where $c_{3}$ is the Euclidean isoperimetric constant at dimension 3. Then $\bigcup_{t \in\left[t_{0}, t_{0}+\epsilon^{2}\right]} B_{t}\left(x_{0}(t), \epsilon\right)$ is unscathed, and $|\operatorname{Rm}|(x)<\frac{\alpha}{\mathfrak{t}(x)-t_{0}}+\epsilon^{-2}$ holds for all $x \in \bigcup_{t \in\left[t_{0}, t_{0}+\epsilon^{2}\right]} B_{t}\left(x_{0}(t), \epsilon\right)$.

As the proof has a lot in common with that of Perelman's pseudolocality theorem, we will focus on the differences, especially the places where the generalized heat kernel comes into play, see [KL08, Section 30-34] for details of the parts which we are brief about.

Proof. Without loss of generality, we assume $t_{0}=0$, and $\alpha<\frac{1}{300}$. Suppose the assertion is not true. Then there are sequences $\epsilon_{k} \rightarrow 0$ and $\delta_{k} \rightarrow 0$, and pointed singular Ricci flows $\left(\mathcal{M}_{k},\left(x_{0 k}, 0\right), g_{k}(\cdot)\right)$ which satisfy the hypotheses of the theorem but for which there is a point $x_{k}$ in the unscathed set $\bigcup_{t \in\left[0, \epsilon_{k}^{2}\right]} B_{t}\left(x_{0 k}(t), \epsilon_{k}\right)$ with $|\operatorname{Rm}|\left(x_{k}\right) \geq \alpha t_{k}^{-1}+\epsilon_{k}^{-2}$. By reducing $\epsilon_{k}$ if needed, we may also assume that

$$
|\operatorname{Rm}|(x)<\alpha t_{k}^{-1}+2 \epsilon_{k}^{-2},
$$

for all $x \in \bigcup_{t \in\left[0, \epsilon_{k}^{2}\right]} B_{t}\left(x_{0 k}(t), \epsilon_{k}\right)$. We abbreviate $d_{t}\left(x_{0 k}(t), x\right)$ as $d(x, t)$.

Let $A_{k}=\frac{1}{300 \epsilon_{k}}$. We say a point $y$ is an $\alpha$-large point if $|\operatorname{Rm}|(y) \geq \frac{\alpha}{\mathfrak{t}(y)}$. First, suppose $\mathcal{P}_{k}:=\bigcup_{t \in\left[0, \epsilon_{k}^{2}\right]} B_{t}\left(x_{0 k}(t),\left(2 A_{k}+1\right) \epsilon_{k}\right)$ is unscathed. Then by a point-picking we can find an $\alpha$-large point $\bar{x}_{k} \in \mathcal{P}_{k}, \mathfrak{t}\left(\bar{x}_{k}\right)=\bar{t}_{k}$, such that

$$
|\operatorname{Rm}|(y) \leq 4|\operatorname{Rm}|\left(\bar{x}_{k}\right):=4 Q_{k},
$$

holds for all $\alpha$-large points $y, \mathfrak{t}(y)=s$, with $s \in\left(0, \bar{t}_{k}\right]$ and $d(y, s) \leq d\left(\bar{x}_{k}, \bar{t}_{k}\right)+$ $A_{k} Q_{k}^{-1 / 2}$. By a distance distortion estimate we can show that (6.2) also holds on $P\left(\bar{x}_{k}, \frac{1}{10} A_{k} Q_{k}^{-1 / 2},-\frac{1}{2} \alpha Q_{k}^{-1 / 2}\right)$. 
Next, suppose $\mathcal{P}_{k}$ is scathed. They by Lemma 3.5, we can also find an $\alpha$-large point $\bar{x}_{k} \in \mathcal{P}_{k}$ so that for large $k,(\underline{6.2})$ holds on $P\left(\bar{x}_{k}, \frac{1}{10} A_{k} Q_{k}^{-1 / 2},-\frac{1}{2} \alpha Q_{k}^{-1 / 2}\right)$.

Now let $u_{k}=\left(4 \pi\left(\bar{t}_{k}-t\right)\right)^{-n / 2} e^{-f_{k}}$ be the heat kernel on $\mathcal{M}_{k}$ starting from $\bar{x}_{k}$, and $v_{k}$ be defined by (5.48). Then $v_{k} \leq 0$. The following lemma says that a local integral of $v_{k}$ has a negative upper bound at some time earlier than $\bar{t}_{k}$.

Lemma 6.2. ([KL08, Lemma 33.4]) There is some $\beta>0$ so that for all sufficiently large $k$, there is some $\tilde{t}_{k} \in\left[\bar{t}_{k}-\frac{1}{2} \alpha Q_{k}^{-1}, \bar{t}_{k}\right)$ with $\int_{B_{k}} v_{k} d V_{k} \leq-\beta$, where $B_{k}$ is the time- $\tilde{t}_{k}$ ball of radius $\sqrt{\bar{t}_{k}-\tilde{t}_{k}}$ centered at $\bar{x}_{k}\left(\tilde{t}_{k}\right)$.

We drop the subscript $k$ for a moment and consider a fixed $\mathcal{M}_{k}$ for $k$ large. Let $\phi$ be a smooth non-increasing function on $\mathbb{R}$ such that: $\phi$ is 1 on $(-\infty, 1]$ and 0 on $[2, \infty)$, and $\phi^{\prime \prime} \geq-10 \phi$ and $\left(\phi^{\prime}\right)^{2} \leq 10 \phi$. Put $h(y)=\phi\left(\frac{d(y, \mathfrak{t}(y))+600 \sqrt{\mathfrak{t}(y)}}{10 A \epsilon}\right)$ on $\mathcal{M}_{t \leq \epsilon^{2}}$. Then

$$
\left(\partial_{t}-\Delta\right) h=\frac{1}{10 A \epsilon}\left(d_{t}-\Delta d+\frac{300}{\sqrt{t}}\right) \phi^{\prime}-\frac{1}{(10 A \epsilon)^{2}} \phi^{\prime \prime}
$$

By (6.1) and Lemma 2.20, we get

$$
d_{t}-\Delta d+\frac{300}{\sqrt{t}} \geq 0
$$

for all points $y, t=\mathfrak{t}(y)$, such that $d(y, t)>\epsilon \geq \sqrt{t}$. In particular, if $\phi^{\prime}\left(\frac{d(y, t)+600 \sqrt{t}}{10 A \epsilon}\right) \neq$ 0 , then $9 A \epsilon<d(y, t)<20 A \epsilon$, and hence (6.4) holds at the point. So we have

$$
\left(\partial_{t}-\Delta\right) h \leq \frac{-\phi^{\prime \prime}}{(10 A \epsilon)^{2}} \leq \frac{10 \phi}{(10 A \epsilon)^{2}}
$$

First, for any open domain $\Omega \subset \mathcal{M}_{t}$ with smooth boundary, we can compute that

$$
\partial_{t} \int_{\Omega} h u d_{t} V=\int_{\Omega}\left(\partial_{t} h-\Delta h\right) u d_{t} V+\int_{\partial \Omega}\left(-\frac{\partial h}{\partial \vec{n}} u+\frac{\partial u}{\partial \vec{n}} h\right) d_{t} S
$$

where $\vec{n}$ is the inwards unit normal vector field on $\partial \Omega$. Applying Theorem 5.5 as in Corollary [5.6, and using (6.5), $h \leq 1$, and $|\nabla h| \leq \frac{\phi^{\prime}}{10 A \epsilon}$, we get

$$
\left.\int h u d_{t} V\right|_{t=0} \geq\left.\int h u d_{t} V\right|_{t=\bar{t}}-\frac{\bar{t}}{(A \epsilon)^{2}} \geq 1-A^{-2}
$$

Similarly, we can show

$$
\int-\left.h v d_{t} V\right|_{t=0} \geq \exp \left(\frac{-\tilde{t}}{10(A \epsilon)^{2}}\right) \int-\left.h v d_{t} V\right|_{t=\tilde{t}} \geq\left(1-A^{-2}\right) \int-\left.h v d_{t} V\right|_{t=\tilde{t}} .
$$


Also, replacing the function $h$ by $\bar{h}(y)=\phi\left(\frac{d(y, \mathrm{t}(y))+600 \sqrt{\mathfrak{t}(y)}}{5 A \epsilon}\right)$, we can show for some constant $C>0$,

$$
\int_{B_{0}\left(x_{0}, 10 A \epsilon\right)} u d V \geq 1-C A^{-2}
$$

By some distance distortion estimates using Lemma 2.20, Lemma 2.21 and (6.1) we can establish the following inclusion

$$
B_{\tilde{t}}(\bar{x}(\tilde{t}), \sqrt{\bar{t}-\tilde{t}}) \subset B_{\tilde{t}}\left(x_{0}(\tilde{t}), 9 A \epsilon\right) .
$$

Since $h(\cdot, \tilde{t})=1$ on $B_{\tilde{t}}\left(x_{0}(\tilde{t}), 9 A \epsilon\right)$ and $v \leq 0$, Lemma 6.2 implies $\int-\left.h v d_{t} V\right|_{t=\tilde{t}} \geq \beta$. Hence by (6.8) we get

$$
\int-\left.h v d_{t} V\right|_{t=0} \geq \beta\left(1-A^{-2}\right)
$$

Let $\tilde{u}(x)=h(x) u(x)$ for all $x \in \mathcal{M}_{0}$, and define $\tilde{f}(x)$ by $\tilde{u}=(2 \pi)^{-\frac{n}{2}} e^{-\tilde{f}}$. Then a direct computation shows

$$
\int_{\mathcal{M}_{0}}-h v d_{0} V=\int_{\mathcal{M}_{0}}\left(-\bar{t}|\nabla \tilde{f}|^{2}-\tilde{f}+3\right) \tilde{u} d_{0} V+\int_{\mathcal{M}_{0}}\left(\bar{t}\left(\frac{|\nabla h|^{2}}{h}-R h\right)-h \log h\right) u d_{0} V .
$$

By (6.9) and $-h \log h \leq 1$ when $h \leq 1$, we have

$$
\begin{aligned}
\int_{\mathcal{M}_{0}}-u h \log h d_{0} V & =\int_{B_{0}\left(x_{0}, 20 A \epsilon\right)-B_{0}\left(x_{0}, 10 A \epsilon\right)}-u h \log h d_{0} V \leq \int_{\mathcal{M}_{0}-B_{0}\left(x_{0}, 10 A \epsilon\right)} u d_{0} V \\
& \leq 1-\int_{B_{0}\left(x_{0}, 10 A \epsilon\right)} u d_{0} V \leq C A^{-2} .
\end{aligned}
$$

Seeing also that $\frac{|\nabla h|^{2}}{h} \leq \frac{10}{(10 A \epsilon)^{2}}$, and $R \geq-1$ on $B_{0}\left(x_{0}, 20 A \epsilon\right)$, we can bound the second integral in (6.12) above by $(1+C) A^{-2}+\epsilon^{2}$. This combining with (6.11) implies

$$
\beta\left(1-A^{-2}\right) \leq \int_{\mathcal{M}_{0}}\left(-\bar{t}|\nabla \tilde{f}|^{2}-\tilde{f}+3\right) \tilde{u} d V+(1+C) A^{-2}+\epsilon^{2} .
$$

Put $\widehat{g}=\frac{1}{2 \bar{t}} g(0), \widehat{u}=(2 \bar{t})^{\frac{n}{2}} \tilde{u}$, and define $\widehat{f}$ by $\widehat{u}=(2 \pi)^{-\frac{n}{2}} e^{-\widehat{f}}$. Restoring the subscript $k$, then $\widehat{u}_{k}$ are supported in $B_{0}\left(x_{0 k}, 2\right)$, and by (6.7) we get

$$
\lim _{k \rightarrow \infty} \int_{B_{0}\left(x_{0 k}, 2\right)} \widehat{u}_{k} d \widehat{V}_{k}=1 .
$$

Moreover, (6.14) implies the following for large $k$,

$$
\frac{1}{2} \beta \leq \int_{B_{0}\left(x_{0 k}, 2\right)}\left(-\frac{1}{2}\left|\nabla \widehat{f}_{k}\right|^{2}-\widehat{f}_{k}+3\right) \widehat{u}_{k} d \widehat{V}_{k}
$$


This contradicts with the isoperimetric inequality in the assumption.

\section{GENERALIZED SINGULAR RiCCI FLOW}

\subsection{Generalized singular Ricci flow: the definition and properties.}

In this subsection, we give the definition and some properties of the generalized singular Ricci flow.

Definition 7.1. Let $(M, g)$ be a Riemannian manifold. For any $x \in M$, let

$$
\bar{\rho}(x)=\sup \left\{r>0: B_{g}(x, r) \text { is relatively compact and }|\mathrm{Rm}| \leq r^{-2} \text { on } B_{g}(x, r)\right\} .
$$

Recall $\rho=R_{+}^{-1 / 2}$, it's clear that $\bar{\rho} \leq \rho$.

By replacing the curvature scale $\rho$ by $\bar{\rho}$ in Definition 2.10 , we say a spacetime is weakly 0-complete (resp. forward or backward).

Definition 7.2. A generalized singular Ricci flow is a $\operatorname{Ricci}$ flow spacetime $(\mathcal{M}, g(t))$, which satisfies:

(1) $\mathcal{M}_{0}=M$ is a complete orientable manifold.

(2) $g(t)$ satisfies the Hamilton-Ivey pinching condition (2.4) with $\varphi=\infty$.

(3) $\mathcal{M}$ is forward 0-complete, and weakly backward 0-complete.

(4) For any $x_{0} \in \mathcal{M}$, there exist $N \in \mathbb{N}$ and a sequence of points $\left\{x_{j}\right\}_{j=0}^{N}$ with $\mathfrak{t}\left(x_{j}\right)=t_{j}$, such that $t_{0} \geq t_{1} \geq \cdots \geq t_{N}=0, x_{j}$ survives until $t_{j+1}$, and $\left.x_{j}\left(t_{j+1}\right)\right), x_{j+1}$ are in the same connected component in $\mathcal{M}_{t_{j+1}}$.

(5) For any $x_{0} \in \mathcal{M}$ surviving on $\left[t_{1}, t_{0}\right]$ for some $t_{1}<t_{0}$, and any $A, \epsilon_{\text {can }}>0$, there is $r>0$ such that the $\epsilon_{\text {can }}$-canonical neighborhood assumption holds at scales less than $r$ on $B_{t}\left(x_{0}(t), A\right)$ for all $t \in\left[t_{1}, t_{0}\right]$.

Definition 7.3. A semi-generalized singular Ricci flow is a Ricci flow spacetime $\left(\mathcal{M}, g(t), x_{0}\right)$ with $\mathfrak{t}(\mathcal{M})=\left[0, t_{0}\right)$ for some $t_{0} \in[0, \infty]$, and $x_{0} \in \mathcal{M}_{0}$, which satisfies the following properties:

(1) $x_{0}$ survives until $t$ for all $t \in\left[0, t_{0}\right)$, .

(2) $g(t)$ satisfies the Hamilton-Ivey pinching condition (2.4) with $\varphi=\infty$.

(3) $\mathcal{M}$ is weakly backward 0-complete.

(4) $\mathcal{M}_{t}$ is connected for each $t \in\left[0, t_{0}\right)$.

(5) For any $t_{1} \in\left[0, t_{0}\right)$, and any $A, \epsilon_{\text {can }}>0$ there is $r>0$ such that the $\epsilon_{\text {can }}{ }^{-}$ canonical neighborhood assumption holds at scales less than $r$ on $B_{t}\left(x_{0}(t), A\right)$ for all $t \in\left[0, t_{1}\right]$.

Remark 7.4. Note that a singular Ricci flow satisfies (11)(21)(31)(41)(51), so it is a generalized singular Ricci flow. Moreover, let $\mathcal{M}$ be a singular Ricci flow, $x_{0} \in \mathcal{M}_{0}$. Suppose $x_{0}$ survives on $\left[0, t_{0}\right)$ for some $t_{0} \in[0, \infty]$, and let $\mathcal{M}_{x_{0}}=\bigcup_{\left[0, t_{0}\right)} \bigcup_{A>0} B_{t}\left(x_{0}(t), A\right)$. By the component stability [KL17, Prop 5.17], the connected components are preserved when going backwards in time, it is clear that $\mathcal{M}_{x_{0}}$ is a semi-generalized singular Ricci flow. 
The following properties can be derived directly from the definition of the semigeneralized singular Ricci flow.

Lemma 7.5. Let $\left(\mathcal{M}, g(t), x_{0}\right)$ be a semi-generalized singular Ricci flow on $\left[0, t_{0}\right)$. Let $t \in\left(0, t_{0}\right)$, then

(i) For any $A>0$, the scalar curvature is proper on $\overline{B_{t}\left(x_{0}(t), A\right)}$.

(ii) For any $A>0$, there exist $\bar{Q}, C>0$ such that for any $x \in B_{t}\left(x_{0}(t), A\right)$, letting $Q=\max \{\bar{Q}, R(x)\}$, then $R \leq C Q$ in $P\left(x,(C Q)^{-1 / 2},-(C Q)^{-1}\right)$, which is contained in $\bigcup_{s \in[0, t]} B_{s}\left(x_{0}(s), 2 A\right)$.

Proof. For any $C>0$, consider the subset $K:=\overline{B_{t}\left(x_{0}(t), A\right)} \cap\{y \in \mathcal{M}: R(y) \leq C\}$, equipped with the metric induced by the length metric on $\overline{B_{t}\left(x_{0}(t), A\right)}$. On the one hand, Lemma 3.1 implies that $B_{t}\left(x_{0}(t), A\right)$ is totally bounded. So $K$ is totally bounded. On the other hand, by the gradient estimate there exists $c>0$ such that for any $x \in K$, the ball $B_{t}(x, c)$ is unscathed and $R \leq 2 C$ in $B_{t}(x, c)$. From this it is easy see that $K$ is complete as a metric space. So $K$ is compact, which established (i).

For any $A>0$, by the gradient estimate, and the distance distortion estimate, and seeing that $\mathcal{M}$ is weakly backward 0-complete, we can find $\bar{Q}, C>0$ such that $(C \bar{Q})^{-1}<t / 2$, and the following holds: For any $x \in B_{t}\left(x_{0}(t), A\right), Q=\max \{\bar{Q}, R(x)\}$, $x$ survives on $\left[t-(C Q)^{-1}, t\right]$, and $R \leq C Q$ in $B_{s}\left(x(s),(C Q)^{-1 / 2}\right)$, which is contained in $B_{s}\left(x_{0}(s), 2 A\right)$. By another distance distortion estimate this implies assertion (ii).

The next proposition says that the connected components of a Ricci flow spacetime are preserved when going backwards in time, assuming the spacetime is weakly backward 0-complete and satisfies a distance-dependent canonical neighborhood assumption. In particular, this component stability holds for generalized singular Ricci flows.

Proposition 7.6. (Component stability when going backwards in time) Let $\mathcal{M}$ be a Ricci flow spacetime, $x_{0}, x_{1} \in \mathcal{M}_{t_{1}}$ for some $t_{1}>0$. Suppose that $\mathcal{M}$ is weakly backward 0-complete. Suppose both $x_{0}, x_{1}$ survive until some $t_{2}<t_{1}$. Suppose also for any $A, \epsilon_{\text {can }}>0$ there exists $r>0$ such that the $\epsilon_{\text {can }}$-canonical neighborhood assumption holds in $B_{t}\left(x_{0}(t), A\right)$ at scales less than $r$ for all $t \in\left[t_{2}, t_{1}\right]$.

Suppose $x_{0}, x_{1}$ are in the same connected component of $\mathcal{M}_{t_{1}}$. Then $x_{0}\left(t_{2}\right), x_{1}\left(t_{2}\right)$ are in the same connected component of $\mathcal{M}_{t_{2}}$.

Proof. Without loss of generality we may assume that $x_{0}(t), x_{1}(t)$ are in the same connected component of $\mathcal{M}_{t}$ for all $t \in\left(t_{2}, t_{1}\right]$. Put

$$
\rho_{0}=\min \left\{\inf _{\left[t_{2}, t_{1}\right]} \bar{\rho}\left(x_{0}(t)\right), \inf _{\left[t_{2}, t_{1}\right]} \bar{\rho}\left(x_{1}(t)\right), 1\right\}>0 .
$$


So by the distance distortion estimate we can find $A>0$ such that $d_{t}\left(x_{0}(t), x_{1}(t)\right) \leq A$ for all $t \in\left(t_{2}, t_{1}\right]$. Fix a small $\delta>0$ and let $C_{0}(\delta)>0$ and $\epsilon_{\text {can }}(\delta)>0$ be from Lemma 2.16. Choose some $\bar{r} \in\left(0, C_{0}^{-1} \rho_{0}\right)$ such that the $\epsilon_{c a n}$-canonical neighborhood assumption holds in $B_{t}\left(x_{0}(t), 6 A\right)$ at scales less than $4 \bar{r}$ for all $t \in\left[t_{2}, t_{1}\right]$. We may also assume $t_{1}-t_{2}<c$ for some $c(\bar{r}, A)>0$ whose value will be determined in the course of the proof.

By Lemma 3.2, there exists a minimizing geodesic $\sigma:[0,1] \rightarrow \mathcal{M}_{t_{1}}$ between $x_{0}$ and $x_{1}$. Choose a division of $[0,1]$ by $0=\alpha_{0} \leq \alpha_{1} \leq \ldots \leq \alpha_{m}=1$ such that one of the following two cases holds for each $i=0,1, \ldots, m-1$ :

(1) $\rho(x) \geq \bar{r}$ for all $x \in \sigma_{i}:=\sigma\left(\left[\alpha_{i}, \alpha_{i+1}\right]\right)$;

(2) $\rho(x) \leq 2 \bar{r}$ for all $x \in \sigma\left(\left[\alpha_{i}, \alpha_{i+1}\right]\right)$, and $\rho\left(\sigma\left(\alpha_{i}\right)\right)=\rho\left(\sigma\left(\alpha_{i+1}\right)\right)=2 \bar{r}$.

Next, suppose by induction that for $\sigma_{i-1}, i \geq 1$, the following assumptions hold:

(a) $\sigma_{i-1}$ survives backwards until $t_{2}$.

(b) $\sigma_{i-1}(t) \subset B_{t}\left(x_{0}(t), 6 A\right)$ for all $t \in\left[t_{2}, t_{1}\right]$.

Suppose $\sigma_{i}$ satisfies case (1) and assume $c$ sufficiently small. Then by the gradient estimate, the distance distortion estimate, and the weakly backward 0-completeness of $\mathcal{M}$, we get that (a)(b) hold for $\sigma_{i}$. In particular, (a)(b) hold for $\sigma_{0}$. So we can assume $\sigma_{i}$ satisfies case $(2), i \geq 1$. Let $t_{3}$ be the infimum of all times $t \in\left[t_{2}, t_{1}\right]$ such that $\sigma_{i}$ survives until $t$, and $\sigma_{i}(s) \subset B_{s}\left(x_{0}(s), 6 A\right)$ for all $s \in\left[t, t_{1}\right]$.

First, since $\bar{r}<C_{0}^{-1} \rho_{0}$ and $\sigma$ is a minimizing geodesic, it follows from Lemma 2.16 that $y_{1}:=\sigma\left(\alpha_{i}\right)$ and $y_{2}:=\sigma\left(\alpha_{i+1}\right)$ are both centers of $\delta$-necks. Taking $c$ small, then by the gradient estimate we have for all $t \in\left(t_{3}, t_{1}\right]$ that $\rho\left(y_{1}(t)\right), \rho\left(y_{2}(t)\right) \in[\bar{r}, 4 \bar{r}]$, and $\rho(x(t)) \leq 4 \bar{r}$ for all $x \in \sigma_{i}$. Since the $\epsilon_{\text {can }}$-canonical neighborhood assumption holds in $B_{t}\left(x_{0}(t), 6 A\right)$ at scales less than $4 \bar{r}$, by Lemma 3.4 that $y_{1}(t), y_{2}(t)$ are centers of $2 \delta$-necks when $c$ is taken sufficiently small. Moreover, by [MT09, Proposition 19.21] we know that $\sigma_{i}(t)$ is contained in a $2 \delta$-tube or a capped $2 \delta$-tube. Since $y_{1}(t), y_{2}(t)$ are the centers of $2 \delta$-necks, it is easy to see that $\sigma_{i}(t)$ is contained in a $2 \delta$-tube. Then the evolution equation of scalar curvature implies $\partial_{t} R(x(t))>0$ for all $x \in \sigma_{i}$ and $t \in\left(t_{3}, t_{1}\right]$. Therefore, $\sigma_{i}$ survives until $t_{3}$.

Next, for any $t \in\left[t_{3}, t_{1}\right]$, let $\mathcal{T}_{t} \subset \mathcal{M}_{t}$ be a $2 \delta$-tube that contains the $100 \bar{r}$ neighborhood of $\sigma_{i}(t)$, and let $\widehat{d}_{t}$ denote the length metric induced by $g(t)$ in $\mathcal{T}_{t}$. Then for any $z_{1}, z_{2} \in \sigma_{i}(t), \widehat{d}_{t}\left(z_{1}, z_{2}\right)$ is realized by a smooth geodesic in $\mathcal{T}_{t}$. Let $\gamma_{t}$ be such a minimizing geodesic connecting $y_{1}(t)$ and $y_{2}(t)$. Then all the second variations along $\gamma_{t}$ are non-negative since it has the minimal length among all smooth curves in a neighborhood around it. So a distance distortion estimate as Lemma 2.20 shows

$$
\partial_{t} \widehat{d}_{t}\left(y_{1}(t), y_{2}(t)\right) \geq-C \bar{r}^{-1},
$$

for some universal constant $C>0$. Integrating this and taking $c$ sufficiently small, we get

$$
\widehat{d}_{t}\left(y_{1}(t), y_{2}(t)\right) \leq \widehat{d}_{t_{1}}\left(y_{1}, y_{2}\right)+C \bar{r}^{-1}\left(t_{1}-t\right) \leq 2 A \text {. }
$$


Moreover, since $\rho(x) \leq 4 \bar{r}$ for all $x \in \sigma_{i}(t)$, by the triangle inequality we get

$$
\widehat{d}_{t}\left(y_{1}(t), x\right) \leq \widehat{d}_{t}\left(y_{1}(t), y_{2}(t)\right)+10 \cdot 2 \cdot 4 \bar{r} \leq 3 A .
$$

By the distance distortion estimate we get $d_{t}\left(x_{0}(t), y_{1}(t)\right) \leq 2 A$ and

$$
d_{t}\left(x, x_{0}\right) \leq d_{t}\left(x_{0}(t), y_{1}(t)\right)+d_{t}\left(y_{1}(t), x\right) \leq 2 A+\widehat{d}_{t}\left(y_{1}(t), x\right) \leq 5 A .
$$

So $\sigma_{i}(t) \subset B_{t}\left(x_{0}(t), 6 A\right)$ for all $t \in\left[t_{3}, t_{1}\right]$. By the infimum assumption of $t_{3}$, we get $t_{3}=t_{2}$. Hence (a)(b) hold for $\sigma_{i}$. So by induction the entire $\sigma$ survives backwards until $t_{2}$. It follows that $x_{0}\left(t_{2}\right), x_{1}\left(t_{2}\right)$ are in the same connected component of $\mathcal{M}_{t_{2}}$.

The following corollary of Proposition 7.6 gives the relation between a semi-generalized singular Ricci flow and a generalized singular Ricci flow.

Corollary 7.7. A Ricci flow spacetime $(\mathcal{M}, g(t))$ is a generalized singular Ricci flow if and only if it satisfies property (11)(21)(3) (41) in Definition 7.2, and for any $x_{0} \in \mathcal{M}$, which survives on $\left[t_{1}, t_{0}\right)$, let $\mathcal{M}_{x_{0}}=\bigcup_{t \in\left[t_{1}, t_{0}\right)} \bigcup_{A>0} B_{t}\left(x_{0}(t), A\right)$, then $\left(\mathcal{M}_{x_{0}}, x_{0}\right)$ is a semi-generalized singular Ricci flow.

Proof. The 'if' part is obvious by the definitions. For the 'only if' part, we need to show $\mathcal{M}_{x_{0}}$ is a semi-generalized singular Ricci flow. It suffices to show $\mathcal{M}_{x_{0}}$ is weakly backward 0-complete. Let $x \in \mathcal{M}_{x_{0}}$ be an arbitrary point. Suppose $\mathfrak{t}(x)=t_{1}$, $x$ survives on $\left(t_{2}, t_{1}\right]$ in $\mathcal{M}_{x_{0}}$ for some $t_{2}<t_{1}$, and $\inf _{t \in\left(t_{2}, t_{1}\right]} \bar{\rho}(x(t))>0$. Since $\mathcal{M}$ is weakly backward 0-complete, $x\left(t_{2}\right)=\lim _{t \searrow t_{2}} x(t)$ exists. By Proposition 7.6, $x\left(t_{2}\right) \in \mathcal{M}_{x_{0}}$. So $\mathcal{M}_{x_{0}}$ is weakly backward 0-complete.

\subsection{Compactness and existence theorems.}

First, we show a compactness theorem which gives a criterion for a sequence of singular Ricci flows to have a subsequence that converges to a semi-generalized singular Ricci flow. Then we apply the compactness theorem to show the existence of generalized singular Ricci flows.

Definition 7.8. (Partial convergence) We say a sequence of Ricci flow spacetimes $\left(\mathcal{M}_{i}, G_{i}\right)$ partially converges to a spacetime $(\mathcal{M}, G)$ if the following holds: There is a sequence of diffeomorphisms $\phi_{i}: U_{i} \rightarrow V_{i} \subset \mathcal{M}_{i}$, where $U_{i}$ and $V_{i}$ are open domains in $\mathcal{M}$ and $\mathcal{M}_{i}$ respectively, such that given any compact subset $K \subset \mathcal{M}, k \in \mathbb{N}$ and $\epsilon>0$, we have $K \subset U_{i}$ for all large $i$, and $\left\|\phi_{i}^{*} G_{i}-G\right\|_{C^{k}(K, G)} \leq \epsilon$.

Let $x_{0} \in \mathcal{M}$ and $x_{0 i} \in \mathcal{M}_{i}$. We say the sequence of pointed spacetimes $\left(\mathcal{M}_{i}, x_{0 i}\right)$ partially converges to $\left(\mathcal{M}, x_{0}\right)$, if $x_{0} \in U_{i}, x_{0 i} \in V_{i}$, and $\phi_{i}\left(x_{0}\right)=x_{0 i}$.

Theorem 7.9 (Compactness theorem). Let $\left\{\left(\mathcal{M}_{i}, x_{0 i}\right)\right\}_{i=1}^{\infty}$ be a sequence of singular Ricci flows, $x_{0 i} \in \mathcal{M}_{i}, \mathfrak{t}\left(x_{0 i}\right)=t_{0 i}$. Suppose

(a) For some $r_{0}>0, x_{0 i}$ survives until $t_{0 i}+r_{0}^{2}$, and $|\mathrm{Rm}| \leq r_{0}^{-2}$ on $P\left(x_{0 i}, r_{0}, r_{0}^{2}\right)$.

(b) For any $\epsilon_{\text {can }}>0$ and $A>0$, there exist $r\left(A, \epsilon_{\text {can }}\right)$ and $\kappa(A)$ such that the

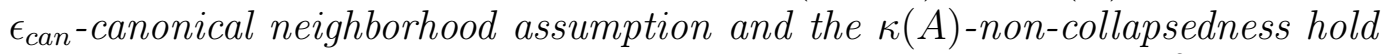
at scales less than $r\left(A, \epsilon_{\text {can }}\right)$ in $B_{t}\left(x_{0 i}(t), A\right)$ for all $t \in\left[t_{0 i}, t_{0 i}+r_{0}^{2}\right]$. 
Then there exists a semi-generalized singular Ricci flow $\left(\mathcal{M}, x_{0}\right)$ on $\left[0, r_{0}^{2}\right), x_{0} \in$ $\mathcal{M}_{0}$, such that a subsequence of $\left(\mathcal{M}_{i, t \geq t_{0 i}}, x_{0 i}\right)$ partially converges to $\left(\mathcal{M}, x_{0}\right)$.

Proof to Theorem 7.9. We may assume $t_{0 i}=0$ and $r_{0} \leq 1$ without loss of generality. Let $d_{G_{i}}$ be the length metric on $\mathcal{M}_{i}$ induced by the spacetime meric $G_{i}$. For any $A>0$, restrict the metric $d_{G_{i}}$ on the subset

$$
\mathcal{P}_{i}(A)=\bigcup_{t \in\left[0, r_{0}^{2}\right)} B_{t}\left(x_{0 i}(t), A\right) \cap\left\{x \in \mathcal{M}_{i}:|\operatorname{Rm}|(x) \leq A^{2}\right\} .
$$

Then the diameter of every $\left(\mathcal{P}_{i}(A), d_{G_{i}}\right)$ is bounded above by $2\left(A+r_{0}^{2}\right)$. Moreover, the following lemma shows that they are uniformly totally bounded.

Lemma 7.10. For any $A, \epsilon>0$, there exists $N=N(A, \epsilon) \in \mathbb{N}$ such that for all $i$, any $\epsilon$-separating subset in $\left(\mathcal{P}_{i}(A), d_{G_{i}}\right)$ has at most $N$ elements.

Proof. On the one hand, by a combination of assumption (b), the gradient estimate and the distance distortion estimate, we may assume that $\epsilon$ is sufficiently small (depending on $A$ ) so that the following holds: First, for any $x \in \mathcal{P}_{i}(A), \mathfrak{t}(x) \geq \epsilon / 8$, the backward parabolic neighborhood $P\left(x, \epsilon / 8,-(\epsilon / 8)^{2}\right)$ is unscathed and contained in $\mathcal{P}_{i}(2 A)$. Second, for any $x \in \mathcal{P}_{i}(A)$ with $\mathfrak{t}(x) \leq \epsilon / 8, x$ survives until 0 , and $x(0) \in \mathcal{P}_{i}(2 A)$. Furthermore, there exists $c(A, \epsilon)>0$ such that

$$
\operatorname{vol}_{G_{i}}\left(P\left(x, \epsilon / 8,-(\epsilon / 8)^{2}\right)\right) \geq c(A, \epsilon) .
$$

On the other hand, assumption (b) allows us to apply Lemma 3.1 on each timeslice $\mathcal{M}_{i, t}$, and deduce that $B_{t}\left(x_{0 i}(t), 2 A\right)$ is uniformly totally bounded, and there is a constant $v(A)>0$ such that

$$
\operatorname{vol}_{g_{i}(t)} B_{t}\left(x_{0 i}(t), 2 A\right) \leq v(A)
$$

for all $i$ and $t \in\left[0, r_{0}^{2}\right)$. Integrating this we get

$$
\operatorname{vol}_{G_{i}}\left(\mathcal{P}_{i}(2 A)\right) \leq \int_{0}^{r_{0}^{2}} \operatorname{vol}_{g_{i}(t)} B_{t}\left(x_{0 i}(t), 2 A\right) d t \leq v(A)
$$

Now suppose $\left\{x_{k}\right\}_{k=1}^{N_{i}}$ is an $\epsilon$-separating subset of $\left(\mathcal{P}_{i}(A), d_{G_{i}}\right)$, and $t_{k}=\mathfrak{t}\left(x_{k}\right)$. Let $\left\{x_{k_{j}}\right\}_{j=1}^{J_{i}}$ be all $x_{k}$ with $t_{k}<\epsilon / 8$, then each $x_{k_{j}}$ survives backwards until 0 and $x_{k_{j}}(0) \in B_{0}\left(x_{0 i}, 2 A\right)$. Since $d_{G_{i}}\left(x_{k_{j}}, x_{k_{l}}\right)>\epsilon$ for any $j \neq l$, by the triangle inequality, $\left\{x_{k_{j}}(0)\right\}_{j=1}^{J_{i}}$ is an $3 \epsilon / 4$-separating subset of $B_{0}\left(x_{0 i}, 2 A\right)$. Since $B_{0}\left(x_{0 i}, 2 A\right)$ is uniformly totally bounded, there is $C(A, \epsilon)>0$ such that $J_{i} \leq C$. Therefore, in order to bound $N_{i}$ we may assume that $t_{k} \geq \epsilon / 8$ for all $k$.

Then each $P\left(x_{k}, \epsilon / 8,-(\epsilon / 8)^{2}\right)$ is unscathed, and $d_{G_{i}}\left(x_{k}, y\right) \leq \epsilon / 8+(\epsilon / 8)^{2}<\epsilon / 4$ for all $y \in P\left(x_{k}, \epsilon / 8,-(\epsilon / 8)^{2}\right)$. Since $d_{G_{i}}\left(x_{k}, x_{j}\right)>\epsilon$ for any $k \neq j$, by the triangle inequality, we see that $P\left(x_{k}, \epsilon / 8,-(\epsilon / 8)^{2}\right), k=1,2, \ldots, N_{i}$, are pairwise disjoint. Therefore, combining (7.7) and (7.9), we conclude that there is $N(A, \epsilon)>0$ such that $N_{i} \leq N(A, \epsilon)$ for all $i$. 
Now since $\left(\mathcal{P}_{i}(A), d_{G_{i}}\right)$ have uniformly bounded diameter and are uniformly totally bounded for all $i$, by Gromov's compactness theorem [Pet06, Proposition 44], we may assume $\left(\mathcal{P}_{i}(A), d_{G_{i}}, x_{0 i}\right)$ converges to a metric space $\left(X(A), d_{A}, x_{0}\right)$ in the pointed Gromov-Hausdorff sense. Since for any $A_{1} \geq A_{2},\left(\mathcal{P}_{i}\left(A_{2}\right), d_{G_{i}}, x_{0 i}\right)$ isometrically embeds into $\left(\mathcal{P}_{i}\left(A_{1}\right), d_{G_{i}}, x_{0 i}\right)$, we get $\left(X\left(A_{2}\right), d_{A_{2}}, x_{0}\right)$ isometrically embeds into $\left(X\left(A_{1}\right), d_{A_{1}}, x_{0}\right)$. Let $(X, d)=\bigcup_{A>0}\left(X(A), d_{A}\right)$, and $\mathcal{N}_{i}=\bigcup_{A>0} \mathcal{P}_{i}(A) \subset \mathcal{M}_{i}$, then $\left(\mathcal{N}_{i}, d_{G_{i}}, x_{0 i}\right)$ converges to $\left(X, d, x_{0}\right)$ in the pointed Gromov-Hausdorff sense as $i \rightarrow \infty$.

Let $x \in X$, and suppose $x \in X(A)$ for some $A>0$. We say $x$ is a smooth point if there are a $\delta>0$, and a sequence of points $x_{i_{k}} \in \mathcal{P}_{i_{k}}(A)$ with $|\operatorname{Rm}|\left(x_{i_{k}}\right) \leq \delta^{-2}$ converging to $x$ (modulo the Gromov-Hausdorff approximations). By the canonical neigborhood assumption in $\mathcal{P}_{i_{k}}(A)$ and the gradient estimate, we can find $\bar{\delta}=\bar{\delta}(\delta, A)$ such that $|\mathrm{Rm}| \leq \bar{\delta}^{-2}$ in $P\left(x_{i_{k}}, \bar{\delta},-\bar{\delta}^{2}\right)$. Moreover, by the non-collapsing assumption in $\mathcal{P}_{i_{k}}(A)$, and the pseudolocality theorem, we get $|\mathrm{Rm}| \leq \bar{\delta}^{-2}$ in $P\left(x_{i_{k}}, \bar{\delta}, \bar{\delta}^{2}\right)$ with a possibly smaller $\bar{\delta}$. Let $U\left(x_{i_{k}}, \bar{\delta}\right)=P\left(x_{i_{k}}, \bar{\delta},-\bar{\delta}^{2}\right) \cup P\left(x_{i_{k}}, \bar{\delta}, \bar{\delta}^{2}\right)$, then by Shi's derivative estimate, the derivatives of $\mathrm{Rm}$ is also uniformly bounded in $U\left(x_{i_{k}}, \bar{\delta}\right)$. So we obtain a smooth limit of $U\left(x_{i_{k}}, \bar{\delta}\right)$ in the Cheeger-Gromov sense by passing to a subsequence. This defines a Ricci flow spacetime metric in a neighborhood of $x$ in $X$, which is isometric to that on $X$ by the uniqueness of the Gromov-Hausdorff limit.

Let $X_{0} \subset X$ be the set of all smooth points. Then we obtain a global Ricci flow spacetime metric on $X_{0}$, denoted by $G_{\infty}=d t^{2}+g(t)$. In particular, $P\left(x_{0 i}, r_{0}, r_{0}^{2}\right)$ converges smoothly to $P\left(x_{0}, r_{0}, r_{0}^{2}\right) \subset X_{0}$. Moreover, by a standard gluing argument (see e.g. [Pet06, Theorem 72]) we get a sequence of diffeomorphisms under which a subsequence of $\mathcal{M}_{i}$ partially converges to $X_{0}$, in the sense of Definition 7.8 ,

It implies that for any $\epsilon_{c a n}, A>0$, there exists $r\left(A, \epsilon_{\text {can }}\right)>0$ such that the

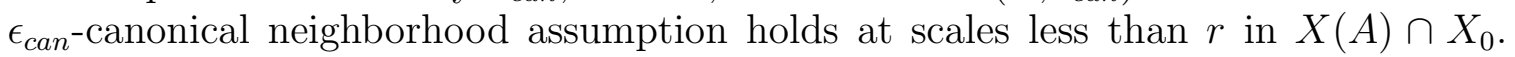
Furthermore, for any $A, C>0$, there exists $c=c(A, C)>0$ such that for all $x \in B_{t}\left(x_{0 i}(t), A\right) \subset \mathcal{N}_{i}$ with $|\operatorname{Rm}|(x) \leq C$, the backward parabolic neighborhood $P\left(x, c,-c^{2}\right)$ in $\mathcal{N}_{i}$ is unscathed. So for all $x \in X(A) \cap X_{0}$ with $|\operatorname{Rm}|(x) \leq C$, the region $P\left(x, c,-c^{2}\right)$ in $X_{0}$ is unscathed. From this it is easy to see that $X_{0}$ is weakly backward 0-complete.

Let $\mathcal{M}=\bigcup_{t \in\left[0, r_{0}^{2}\right)} \bigcup_{A>0} B_{t}\left(x_{0}(t), A\right)$ be a subset in $X_{0}$. Then $\mathcal{M}$ is a smooth Ricci flow spacetime with connected time-slices, and a subsequence of $\mathcal{M}_{i}$ partially converge to $\mathcal{M}$. It is clear that $\mathcal{M}$ satisfies property (11)(2) (4) (15) in Definition 7.3 . Moreover, applying Proposition 7.6 to $X_{0}$, we see that $\mathcal{M}$ is also weakly backward 0 -complete and hence satisfies property (3). This proved Theorem 7.9 .

The next lemma shows that the convergence of the initial manifolds implies the convergence of the singular Ricci flows to some semi-generalized singular Ricci flow. 
Lemma 7.11. Let $\left(\mathcal{M}_{i}, g_{i}(t)\right)$ be a sequence of singular Ricci flows. Suppose for some $t_{1} \geq 0$ and $x_{0 i} \in \mathcal{M}_{i, t_{1}}$, the sequence of time-slices $\left(\mathcal{M}_{i, t_{1}}, g_{i}\left(t_{1}\right), x_{0 i}\right)$ smoothly converges to a 0-complete manifold $\left(M, g, x_{0}\right)$. Then there exists a semi-generalized singular Ricci flow $\left(\mathcal{M}, g(t), x_{0}\right)$ on $\left[0, t_{0}\right)$ for some $t_{0}>0$, such that $\mathcal{M}_{0}=M$ and a subsequence of $\left(\mathcal{M}_{i, t \geq t_{1}}, x_{0 i}\right)$ partially converges to $\left(\mathcal{M}, x_{0}\right)$. Moreover, $t_{0}$ can be chosen such that $\inf _{\left[0, t_{0}\right)} \rho\left(x_{0}(t)\right)=0$, i.e. $\limsup _{t \nearrow t_{0}}|\operatorname{Rm}|\left(x_{0}(t)\right)=\infty$.

Proof. Without loss of generality we may assume $t_{1}=0$. On the one hand, by the pseudolocality theorem for singular Ricci flow, Theorem 6.1, there exist $r_{0}, t_{0}>0$ such that for all large $i$ the domain $P\left(x_{0 i}, r_{0}, t_{0}\right) \subset \mathcal{M}_{i}$ is unscathed and $|\mathrm{Rm}| \leq r_{0}^{-2}$ holds there. Moreover, for any fixed $A>0$, there exists $t_{A} \in\left(0, t_{0}\right)$ such that the geometry $P\left(x_{0 i}, 2 A, t_{A}\right) \subset \mathcal{M}_{i}$ is uniformly bounded for all large $i$. By a distance distortion estimate this implies the uniformly bounded geometry on $\bigcup_{t \in\left[0, t_{A}\right]} B_{t}\left(x_{0 i}(t), A\right) \subset \mathcal{M}_{i}$ for a possibly smaller $t_{A}$.

On the other hand, for any $t \in\left(t_{A}, t_{0}\right)$, by Proposition 4.1 there are constants $r, \kappa>$ 0 , such that the $\epsilon_{\text {can }}$-canonical neighborhood assumption and the $\kappa$-non-collapsing assumption hold at scales less than $r$ in $B_{t}\left(x_{0 i}(t), A\right)$. So by Theorem 7.9 there is a subsequence of $\left(\mathcal{M}_{i}, x_{0 i}\right)$ which partially converges to $\left(\mathcal{M}, x_{0}\right)$.

If $\inf _{\left[0, t_{0}\right)} \rho\left(x_{0}(t)\right)>0$, then by Lemma 4.6 we have $\inf _{\left[0, t_{0}\right)} \bar{\rho}\left(x_{0}(t)\right)>0$. So there exist $\kappa^{\prime}, r^{\prime}>0$ such that $\mathcal{M}$ is $\kappa^{\prime}$-non-collapsed at $x_{0}(t)$ at scales less than $r^{\prime}$ for all $t \in\left[0, t_{0}\right)$. Repeating the above argument at $t$ sufficiently close to $t_{0}$, we can extend $\mathfrak{t}(\mathcal{M})$ to $\left[0, t_{1}\right)$ with $t_{1}>t_{0}$. So we may assume $\inf _{\left[0, t_{0}\right)} \rho\left(x_{0}(t)\right)=0$.

Theorem 7.12. (Existence of a semi-generalized singular Ricci flow) Let $(M, g)$ be a $3 d$ orientable complete Riemannian manifold, $x_{0} \in M$. Then there exists a semigeneralized singular Ricci flow $\left(\mathcal{M}, g(t), x_{0}\right)$ on $\left[0, t_{0}\right)$ for some $t_{0}>0$ with $\mathcal{M}_{0}=M$.

Moreover, if $M$ is the double cover of a non-orientable manifold, and $\sigma: M \rightarrow M$ is the corresponding deck transformation which acts as an isometry. Then there is a semi-generalized singular Ricci flow $\mathcal{M}$ with $\mathcal{M}_{0}=M$ such that $\sigma$ extends to an isometry on $\mathcal{M}$, which acts free on the open domain $\{x \in \mathcal{M}: x$ survives until $t=0\}$.

Proof. The first assertion follows directly from Lemma 7.11. It only remains to establish the assertion about the $\mathbb{Z}_{2}$-symmetry. For this we assume $\left(M, g, x_{0}\right)$ is the double cover of a non-orientable manifold $(N, \bar{g})$, and $\sigma: M \rightarrow M$ is the non-trivial deck transformation in $\mathbb{Z}_{2}$, which acts as an isometry. Let $N_{i} \subset N$ be a compact 3 dimensional submanifold with smooth boundary that contains $B_{\bar{g}}\left(\pi\left(x_{0}\right), i\right)$. Take $i>d_{g}\left(x_{0}, \sigma\left(x_{0}\right)\right)$, then $\pi^{-1}\left(N_{i}\right)$ is a compact connected orientable manifold which has smooth orientable boundary $\pi^{-1}\left(\partial N_{i}\right)$, and $B_{g}\left(x_{0}, i\right) \cup B_{g}\left(\sigma\left(x_{0}\right), i\right) \subset \pi^{-1}\left(N_{i}\right)$. First, we extend $\pi^{-1}\left(N_{i}\right)$ and the metric past a collar of its boundary, and assume the new metric $g_{i}$ is isometric to the product of a metric on $\pi^{-1}\left(\partial N_{i}\right)$ with an interval. Next, since $\pi^{-1}\left(\partial N_{i}\right)$ is $\sigma$-invariant, we can extend the action of $\sigma$ to the collar neighborhood $\pi^{-1}\left(\partial N_{i}\right) \times[0,1]$ such that $\sigma(x, s)=\sigma(x, 0)$ for all $x \in \pi^{-1}\left(\partial N_{i}\right)$ and $s \in[0,1]$. Then by replacing $g_{i}$ with $\frac{1}{2}\left(g_{i}+\sigma^{*} g_{i}\right)$, we may assume $g_{i}$ is $\sigma$-invariant, and it is still a product metric near the new boundary. Therefore, by doubling the extended 
manifold, we get a closed, connected and orientable manifold $\left(M_{i}, g_{i}, x_{0}\right)$ with a deck transformation $\sigma_{i}$ which is an isometry, and $g_{i}=g, \sigma_{i}=\sigma$ on $B_{g_{i}}\left(x_{0}, i\right)$.

Let $\left(\mathcal{M}_{i}, g_{i}(t), x_{0}\right)$ be a sequence of singular Ricci flows starting from $\left(M_{i}, g_{i}, x_{0}\right)$. Then by Lemma 7.11 there is $t_{0}>0$ such that $\left\{\left(\mathcal{M}_{i}, g_{i}(t), x_{0}\right)\right\}$ converges to a semigeneralized singular Ricci flow $\left(\mathcal{M}, x_{0}\right)$ on $\left[0, t_{0}\right)$. Moreover, by the uniqueness of singular Ricci flow in [BK17b], each $\sigma_{i}: M_{i} \rightarrow M_{i}$ can be uniquely extended to an isometry $\sigma_{i}: \mathcal{M}_{i} \rightarrow \mathcal{M}_{i}$. So for any $x_{1}, x_{2} \in M_{i}$, if $\sigma_{i}\left(x_{1}\right)=x_{2}$ and $x_{1}$ survives until $t>0$, then $x_{2}$ also survives until $t$ and $\sigma_{i}\left(x_{1}(t)\right)=\sigma_{i}\left(x_{2}(t)\right)$. Therefore, $\sigma_{i}$ converges to an isometry $\sigma: \mathcal{M} \rightarrow \mathcal{M}$, which acts free on $\left\{x \in \mathcal{M}: x\right.$ survives back to $\mathcal{M}_{0}=$ $M\}$

The next lemma shows that for two spacetimes $\left(\mathcal{N}_{j}, x_{j}\right), j=1,2$, which have connected time-slices, suppose they are limits of a same sequence of Ricci flow spacetimes $\mathcal{M}_{i}$ under the partial convergence. Then they are isometric if the preimages of $x_{1}, x_{2}$ under the diffeomorphisms are contained in a parabolic region in $\mathcal{M}_{i}$.

Lemma 7.13. Let $\mathcal{M}_{i}$ be a sequence of Ricci flow spacetimes, $x_{1, i}, x_{2, i} \in \mathcal{M}_{i, 0}$. Suppose $\left(\mathcal{M}_{i}, x_{j, i}\right)$ partially converges to a Ricci flow spacetime $\left(\mathcal{N}_{j}, x_{j}\right)$ on $[0, T)$, for some $T>0, x_{j} \in \mathcal{N}_{j, 0}$, and each time-slice $\mathcal{N}_{j, t}$ is connected, $j=1,2$. Suppose also there is $D>0$ such that $x_{2, i} \in B_{0}\left(x_{1, i}, D\right)$ for all $i$, and $P\left(x_{1}, D, T-\delta\right) \subset \mathcal{N}_{1}$ is unscathed for any $\delta>0$. Then $\mathcal{N}_{1}$ is isometric to $\mathcal{N}_{2}$.

Proof. Let $H_{i}$ be the spacetime metric of $\mathcal{M}_{i}$, and $G_{j}$ the spacetime metric of $\mathcal{N}_{j}$, $j=1,2$. Let $\phi_{j, i}: \mathcal{N}_{j} \supset U_{j, i} \rightarrow V_{j, i} \subset \mathcal{M}_{i}$ be the two corresponding diffeomorphism sequences such that $\cup_{i=1}^{\infty} U_{j, i}=\mathcal{N}_{j}$ and $\left\|\phi_{j, i}^{*} H_{i}-G_{j}\right\| \leq \epsilon_{i} \rightarrow 0$. Let $P_{j, k}=\bigcup_{t \in\left[0, T-k^{-1}\right]} \overline{B_{t}\left(x_{j}, k\right)} \cap\left\{x: \rho(x) \geq k^{-1}\right\}$, then $\cup_{k=1}^{\infty} P_{j, k}=\mathcal{N}_{j}$. By the assumption of $x_{1, i}$ and $x_{2, i}$, for a given $k$ there exists $\ell(k, D) \in \mathbb{N}$ such that for all large $i$, we have $\phi_{1, i}\left(P_{1, k}\right) \subset \phi_{2, i}\left(P_{2, \ell}\right)$. So the maps $\phi_{2, i}^{-1} \circ \phi_{1, i}: \mathcal{N}_{1} \supset P_{1, k} \rightarrow \mathcal{N}_{2}$ are well-defined, and $\left\|\left(\phi_{2, i}^{-1} \circ \phi_{1, i}\right)^{*} G_{2}-G_{1}\right\| \leq \delta_{i} \rightarrow 0$. Moreover, $\phi_{2, i}^{-1} \circ \phi_{1, i}\left(P_{1, k}\right)$ form an exhaustion of $\mathcal{N}_{2}$ as $i, k \rightarrow \infty$. So $\mathcal{N}_{1}$ is isometric to $\mathcal{N}_{2}$.

Theorem 7.14. (Theorem 1.1 and 1.2, Existence of generalized singular Ricci flow) Let $(M, g)$ be a $3 d$ orientable complete Riemannian manifold, $x_{0} \in M$. Let $\left(\mathcal{M}_{i}, g_{i}(t), x_{0 i}\right)$ be a sequence of singular Ricci flows with $\left(\mathcal{M}_{i, 0}, g_{i}(0), x_{0 i}\right)$ smoothly converging to $\left(M, g, x_{0}\right)$. Then there exists a generalized singular Ricci flow $\mathcal{M}$ with $\mathcal{M}_{0}=M$, such that $\left(\mathcal{M}_{i}, x_{0 i}\right)$ partially converges to $\left(\mathcal{M}, x_{0}\right)$.

Moreover, if $M$ is the double cover of a non-orientable manifold, then the same conclusion as Theorem 7.12 holds.

Proof. Let $x_{0} \in M$, by Lemma 7.11 there exist $t_{0}>0$ and a semi-generalized singular Ricci flow $\left(\mathcal{M}^{1}, x_{0}\right)$ on $\left[0, t_{0}\right)$ such that $\left(\mathcal{M}_{i}, G_{i}, x_{0}\right)$ partially converges to $\left(\mathcal{M}^{1}, x_{0}\right)$ and $\inf _{\left[0, t_{0}\right)} \rho\left(x_{0}(t)\right)=0$.

Suppose by induction that there is a sequence of Ricci flow spacetimes $\left\{\mathcal{M}^{j}\right\}_{j=1}^{k-1}$ such that $\mathcal{M}^{j-1} \subset \mathcal{M}^{j}$ and the followings hold for all $j=2, \ldots, k-1$ : 
(1) A subsequence of $\mathcal{M}_{i}$ partially converges to $\mathcal{M}^{j}$.

(2) $\mathcal{M}^{j}$ is weakly backward 0-complete.

(3) For any $x \in \mathcal{M}^{j-1}$, let $a$ be the supremum of all times $t$ until which $x$ survives until in $\mathcal{M}^{j}$. Then $\inf _{[t(x), a)} \rho(x(t))=0$.

(4) For any $x \in \mathcal{M}^{j-1}$, suppose $x$ survives until some $t>\mathfrak{t}(x)$. Let $\mathcal{M}_{x} \subset \mathcal{M}^{j}$ be the subset $\bigcup_{s \in[\mathfrak{t}(x), t)} \bigcup_{A>0} B_{s}(x(s), A)$, then $\left(\mathcal{M}_{x}, x\right)$ is a semi-generalized singular Ricci flow on $[\mathfrak{t}(x), t)$.

Let $\left\{x_{j}\right\}_{j=1}^{\infty}$ be a dense subset in $\mathcal{M}^{k-1}$. For each $x_{j}$, by Lemma 7.11 there is a subsequence of $\left\{\left(\mathcal{M}_{i}, x_{j}\right)\right\}_{i=1}^{\infty}$ that partially converges to a semi-generalized singular Ricci flow $\left(\mathcal{N}_{j}, x_{j}\right)$, such that $x_{j}$ survives in $\mathcal{N}_{j}$ until $R\left(x_{j}(t)\right)$ goes unbounded. So by a diagonal argument we may assume that $\left\{\left(\mathcal{M}_{i}, x_{j}\right)\right\}_{i=1}^{\infty}$ converges to $\left(\mathcal{N}_{j}, x_{j}\right)$ for all $x_{j}$.

For any $y_{1}, y_{2} \in \mathcal{M}^{k-1} \sqcup \coprod_{j=1}^{\infty} \mathcal{N}_{j}$, we say $y_{1} \sim y_{2}$ if there is a sequence of points $w_{i} \in \mathcal{M}_{i}$ such that modulo the diffeomorphism maps we have $w_{i} \rightarrow y_{1}$ and $w_{i} \rightarrow y_{2}$ as $i \rightarrow \infty$. This defines an equivalent relation in $\mathcal{M}^{k-1} \sqcup \coprod_{j=1}^{\infty} \mathcal{N}_{j}$. If $y_{1} \sim y_{2}$, then by the uniqueness of the smooth limit, there is $\delta>0$ such that the neighborhoods of $P\left(y_{i}, \delta, \delta^{2}\right) \cup P\left(y_{i}, \delta,-\delta^{2}\right), i=1,2$, are unscathed and the spacetime metrics on them are isometric. So there is a well-defined smooth Ricci flow spacetime metric on the quotient space $\mathcal{M}^{k}:=\left(\mathcal{M}^{k-1} \sqcup \coprod_{j=1}^{\infty} \mathcal{N}_{j}\right) / \sim$. So (1) holds for $j=k$.

Since each connected component of $\mathcal{M}_{t}^{k}$ is isometric to either $\mathcal{M}_{t}^{k-1}$ or some $\mathcal{N}_{j, t}$, we get that $\mathcal{M}_{t}^{k}$ is 0 -complete. For any $x \in \mathcal{M}^{k}, \mathfrak{t}(x)=t_{0}$, suppose $x$ survives on $\left(t_{1}, t_{0}\right]$ and $\lim _{t \rightarrow t_{1}} \bar{\rho}(x(t))>0$. Assume $x \in \mathcal{M}^{k-1}$, then since $\mathcal{M}^{k-1}$ is weakly backward 0-complete, it follows that $x(t) \in \mathcal{M}^{k-1}$ and $\lim _{t \rightarrow t_{1}} x(t)$ exists. Otherwise, assume $x \in \mathcal{N}_{j}$ for some $j \in \mathbb{N}$, and let $t_{2} \in\left(t_{1}, t_{0}\right]$ be the infimum of time $t$ such that $x(t) \in \mathcal{N}_{j}$. Then $x\left(t_{2}\right)=\lim _{t \rightarrow t_{2}} x(t)$ exists because $\mathcal{N}_{j}$ is weakly backward 0 complete. If $t_{2}>t_{1}$, then we have $x\left(t_{2}\right) \in \mathcal{N}_{j} \cap \mathcal{M}^{k-1}$, and the existence of $\lim _{t \rightarrow t_{1}} x(t)$ exists by the weakly backward 0 -completeness of $\mathcal{M}^{k-1}$. So $\mathcal{M}^{k}$ is weakly backward 0-complete, and hence (2) holds.

It is clear that (3)(4) hold for each $x_{j}$. We claim that (3)(4) hold for every point in $\mathcal{M}^{k-1}$. To verify (3), let $x \in \mathcal{M}^{k-1}$ be an arbitrary point, $\mathfrak{t}(x)=t_{1}$. Let $t_{2}>t_{1}$ be the supremum of all times until which $x$ survives in $\mathcal{M}^{k}$. Suppose by contradiction that $\inf _{\left[t_{1}, t_{2}\right)} \rho(x(t))>0$. Then by Lemma 7.11 there is $\delta>0$ such that by passing to a subsequence, $\left(\mathcal{M}_{i}, x\right)$ partially converges to a semi-generalized singular Ricci flow $\mathcal{N}$ on $\left[t_{1}, t_{2}+\delta^{2}\right)$, and $P\left(x, \delta, t_{2}-t_{1}+\delta^{2}\right)$ is unscathed. By the density of $\left\{x_{j}\right\}_{j=1}^{\infty}$, there exists $x_{j} \in P\left(x, \delta, \delta^{2}\right) \subset \mathcal{M}^{k-1}$. Then $x_{j}$ survives on $\left[\mathfrak{t}\left(x_{j}\right), t_{2}+\delta^{2}\right)$ in $\mathcal{N}_{j}$. So it follows from Lemma 7.13 that $\mathcal{N}_{j}$ is isometric to $\mathcal{N}$ on $\left[t_{1}+\delta^{2}, t_{2}+\delta^{2}\right)$. In particular, $x \in \mathcal{N}_{j} \subset \mathcal{M}^{k}$ survives until $t_{2}+\delta^{2} / 2$, contradicting with the supremum assumption of $t_{2}$. This verifies (3).

To verify (4), let $x \in \mathcal{M}^{k-1}$ be an arbitrary point, $\mathfrak{t}(x)=t_{1}$, and assume $x$ survives until some $t_{2}>t_{1}$, and $\mathcal{M}_{x}$ is defined as in (4). Choose $\delta>0$ such that $P\left(x, \delta, t_{2}-t_{1}\right)$ is unscathed, and pick some $x_{j} \in P\left(x, \delta, \delta^{2}\right)$ by the density of $\left\{x_{j}\right\}_{j=1}^{\infty}$. Then by 
Lemma 7.13, $\mathcal{M}_{x}$ is isometric to $\mathcal{N}_{j}$ on $\left[t_{1}+\delta^{2}, t_{2}\right)$, and hence $\left(\mathcal{M}_{x}, x\right)$ is a semigeneralized singular Ricci flow on $\left[t_{1}+\delta^{2}, t_{2}\right)$. Letting $\delta \rightarrow 0$, it implies that $\mathcal{M}_{x}$ is a semi-generalized singular Ricci flow on $\left[t_{1}, t_{2}\right)$. This verifies (4).

So by induction we obtain an infinite sequence of spacetimes $\left\{\mathcal{M}^{k}\right\}_{k=1}^{\infty}$ with $\mathcal{M}^{k-1} \subset$ $\mathcal{M}^{k}$, which satisfies all inductive assumptions. Let $\mathcal{M}=\bigcup_{k=1}^{\infty} \mathcal{M}^{k}$, then by passing to a subsequence $\mathcal{M}_{i}$ partially converges to $\mathcal{M}$. By the 'if' part of Corollary 7.7, it is clear that $\mathcal{M}$ is a generalized singular Ricci flow. The assertion about the $\mathbb{Z}_{2^{-}}$ symmetry follows in the same way as Theorem 7.12,

\section{RicCi Flows With NON-NEGative RicCi CURVATURE}

In this section, we prove Theorem 1.3, First, by adapting the maximum principle argument in Che09 and CXZ13 to a generalized singular Ricci flow, we show in Lemma 8.1 and 8.2 that it preserves the non-negativity of scalar curvature and Ricci curvature.

Then we prove Lemma 8.3, which is the last ingredient needed to prove Theorem 1.3. It says that in a 3-dimensional manifold with Ric $\geq 0$, no singularity can form within finite distance along a minimizing geodesic covered by final time-slices of strong $\delta$-necks.

Lemma 8.1. Let $(M, g)$ be a 3 dimensional complete Riemannian manifold with $R \geq 0$. Let $(\mathcal{M}, g(t))$ be a generalized singular Ricci flow starting from $(M, g)$. Then $R \geq 0$ on $\mathcal{M}$.

Proof. By property (4) in Definition 7.2 and Corollary 7.7, it suffices to prove the lemma for a semi-generalized singular Ricci flow $\left(\mathcal{M}, g(t), x_{0}\right)$ on $\left[0, t_{0}\right), x_{0} \in \mathcal{M}_{0}=$ $M$. We may assume that there is $r_{0}>0$ such that $\bigcup_{t \in\left[0, t_{0}\right)} B_{t}\left(x_{0}(t), r_{0}\right)$ is unscathed and $\operatorname{Ric}(x) \leq r_{0}^{-2}$ there. Then by Lemma 2.20, we have

$$
\left(\partial_{t}-\Delta\right) d_{t}\left(x_{0}(t), x\right) \geq-\frac{10}{3} r_{0}^{-1}
$$

for all $x \in \mathcal{M}_{t}$ with $d_{t}\left(x, x_{0}(t)\right)>r_{0}$.

Let $A \geq \frac{80}{3} r_{0}^{-2} t_{0}+2$ and define the following function on $\mathcal{M}$

$$
u(x)=\varphi\left(\frac{d_{t}\left(x_{0}(t), x\right)+\frac{10}{3} r_{0}^{-1} t}{A r_{0}}\right) R(x),
$$

for all $x \in \mathcal{M}_{t}, t \in\left[0, t_{0}\right)$, where we choose $\varphi$ to be a smooth non-negative nonincreasing function such that $\varphi=1$ on $\left(-\infty, \frac{7}{8}\right], \varphi=0$ on $[1, \infty)$ and $\left|\frac{2 \varphi^{\prime 2}}{\varphi}-\varphi^{\prime \prime}\right| \leq$ $C \sqrt{\varphi}$. Then with the choice of $A$, we have $u(x)=R(x)$ for all $x \in B_{t}\left(x_{0}(t), \frac{3}{4} A r_{0}\right)$, and $u(x)=0$ for all $x \in \mathcal{M}_{t} \backslash B_{t}\left(x_{0}(t), A r_{0}\right)$.

Let $u_{\min }(t):=\min \left\{\inf _{\mathcal{M}_{t}} u(\cdot), 0\right\}, t \in\left[0, t_{0}\right)$. If $u_{\min }(t)<0$, we claim that $\inf _{\mathcal{M}_{t}} u(\cdot)$ can be achieved. Suppose not, then there exists a sequence of points 
$x_{i} \in B_{t}\left(x_{0}(t), A r_{0}\right)$ such that $u\left(x_{i}\right) \rightarrow u_{\text {min }}(t)$ as $i \rightarrow \infty$. By Lemma 7.5, the properness of scalar curvature, we may assume that $R\left(x_{i}\right) \rightarrow \infty$. So $u\left(x_{i}\right) \geq 0$ for all large $i$, a contradiction.

Then we claim the following holds for all $t \in\left(0, t_{0}\right)$ :

$$
u_{\min }(t) \leq \lim \inf _{s \searrow t} u_{\min }(s) .
$$

Suppose this is not true at some $t \in\left(0, t_{0}\right)$. Then there exist some $\epsilon>0$ and a sequence of times $s_{i}>t$ which converges to $t$ as $i \rightarrow \infty$ such that

$$
u_{\min }(t)>u_{\min }\left(s_{i}\right)+\epsilon,
$$

for all $i$. Let $x_{i} \in B_{s_{i}}\left(x_{0}\left(s_{i}\right), A r_{0}\right)$ be a point such that

$$
u\left(x_{i}\right) \leq u_{\min }\left(s_{i}\right)+\frac{\epsilon}{2}<u_{\min }(t)-\frac{\epsilon}{2} .
$$

If $R\left(x_{i}\right)$ is not uniformly bounded, then $u\left(x_{i}\right) \geq 0$ for large $i$, which implies $u_{\text {min }}(t) \geq \epsilon>0$, a contradiction. So we may assume $R\left(x_{i}\right)$ is uniformly bounded, and hence by Lemma 7.5 there is a $\delta>0$ such that $R \leq \delta^{-2}$ in $P\left(x_{i}, \delta,-\delta^{2}\right) \subset \subset$ $\bigcup_{t \in\left[0, t_{0}\right)} B_{t}\left(x_{0}(t), 2 A r_{0}\right)$. So $u$ is uniformly continuous on $\bigcup_{i} P\left(x_{i}, \delta,-\delta^{2}\right)$. Since $s_{i}-t \rightarrow 0$, this implies $u\left(x_{i}(t)\right) \leq u\left(x_{i}\right)+\frac{\epsilon}{2}$ for all large $i$. So

$$
u_{\min }(t) \leq u\left(x_{i}(t)\right) \leq u\left(x_{i}\right)+\frac{\epsilon}{2},
$$

which contradicts with (8.5). So claim (8.3) is true.

Now we argue by maximum principle that the following holds for all times:

$$
u_{\min }(t) \geq-\frac{2 C_{0}}{\left(A r_{0}\right)^{2}},
$$

where $C_{0}>0$ will be specified below. Suppose not and let $T$ be the supremum of all times $t$ such that (8.7) is true on $[0, t]$. Then $T>0$ and there exists a sequence $t_{i}>T$ converging to $T$ as $i \rightarrow \infty$ such that $u_{\min }\left(t_{i}\right)<-\frac{2 C_{0}}{\left(A r_{0}\right)^{2}}$. Using inequality (8.3) at $T$, we have that $u_{\min }(T) \leq-\frac{2 C_{0}}{\left(A r_{0}\right)^{2}}<0$.

Since $u_{\min }(T)<0$, there exists $x_{T} \in \mathcal{M}_{T}$ such that $u_{\min }(T)=u\left(x_{T}\right)$. Then by the choice of $T$ it is easy to see the followings hold at $x_{T}: \nabla u=0, \Delta u \geq 0$, and $\partial_{t} u \leq 0$. By a direct computation we get the following at $x_{T}$,

$$
\begin{aligned}
R \nabla \varphi+\varphi \nabla R & =0, \\
2 \nabla \varphi \cdot \nabla R & =-2 \frac{|\nabla \varphi|^{2}}{\varphi} R=-2 \frac{\varphi^{\prime 2}}{\varphi} \frac{1}{\left(A r_{0}\right)^{2}} R .
\end{aligned}
$$

By the evolution equation $\left(\partial_{t}-\Delta\right) R=2|\operatorname{Ric}|^{2}$, we get

$$
\begin{aligned}
\left(\partial_{t}-\Delta\right) u= & \varphi^{\prime} R \frac{1}{A r_{0}}\left[\left(\partial_{t}-\Delta\right) d_{t}\left(x_{0}(t), x\right)+\frac{10}{3} r_{0}^{-1}\right] \\
& -\varphi^{\prime \prime} \frac{1}{\left(A r_{0}\right)^{2}} R+2 \varphi|\operatorname{Ric}|^{2}-2 \nabla \varphi \nabla R
\end{aligned}
$$


restricting which at $x_{T}$ and using (8.1), (8.8) and $3|\mathrm{Ric}|^{2} \geq R^{2}$, we obtain the following

$$
\begin{aligned}
0 \geq\left(\partial_{t}-\Delta\right) u & \geq \frac{2}{3} \varphi R^{2}-\varphi^{\prime \prime} \frac{1}{\left(A r_{0}\right)^{2}} R+2 \frac{\varphi^{\prime 2}}{\varphi} \frac{1}{\left(A r_{0}\right)^{2}} R \\
& \geq \frac{2}{3} \varphi R^{2}-\frac{C}{\left(A r_{0}\right)^{2}} \sqrt{\varphi} R \\
& \geq \frac{1}{3}\left(u_{\min }^{2}(T)-\frac{C_{0}^{2}}{\left(A r_{0}\right)^{4}}\right),
\end{aligned}
$$

where $C_{0}=\frac{3 C}{2}$, and we have used $\left|\frac{2 \varphi^{\prime 2}}{\varphi}-\varphi^{\prime \prime}\right| \leq C \sqrt{\varphi}$, and Cauchy inequality $\frac{C}{\left(A r_{0}\right)^{2}} \sqrt{\varphi} R \leq \frac{1}{3} \varphi R^{2}+\frac{C_{0}^{2}}{3\left(A r_{0}\right)^{4}}$. Since $u_{\min }(T)<0$, (8.10) implies $u_{\min }(T) \geq \frac{-C_{0}}{\left(A r_{0}\right)^{2}}$, a contradiction. So $u_{\min }(t) \geq \frac{-2 C_{0}}{\left(A r_{0}\right)^{2}}$ for all $t \in\left[0, t_{0}\right)$, and in particular it implies

$$
R(x) \geq-\frac{2 C}{\left(A r_{0}\right)^{2}}
$$

for all $x \in B_{t}\left(x_{0}(t), \frac{3}{4} A r_{0}\right), t \in\left[0, t_{0}\right)$. Letting $A$ go to infinity, we get $R(x) \geq 0$, for all $x \in \mathcal{M}$.

Lemma 8.2. Let $(M, g)$ be a 3 dimensional complete Riemannian manifold with Ric $\geq 0$. Let $(\mathcal{M}, g(t))$ be a generalized singular Ricci flow starting from $(M, g)$. Then $\mathrm{Ric} \geq 0$ on $\mathcal{M}$.

Proof. For the same reason as in Lemma 8.1, it suffices to prove the lemma for a semi-generalized singular Ricci flow $\left(\mathcal{M}, g(t), x_{0}\right)$ on $\left[0, t_{0}\right), x_{0} \in M$. We may assume that there is $r_{0}>0$ such that $\bigcup_{t \in\left[0, t_{0}\right)} B_{t}\left(x_{0}(t), r_{0}\right)$ is unscathed and $\operatorname{Ric}(x) \leq r_{0}^{-2}$ there.

Let $\lambda \geq \mu \geq \nu$ be the three eigenvalues of the curvature operator. Then it suffices to show that the following inequality holds on $\mathcal{M}$ for any $a>0$,

$$
R+a(\mu+\nu) \geq 0
$$

In fact, if this is true, then we have $R+\epsilon^{-1}(\mu+\nu) \geq 0$ for any $\epsilon>0$. Multiplying both sides by $\epsilon$ and letting $\epsilon$ go to zero we get $\mu+\nu \geq 0$, i.e. Ric $\geq 0$.

Now suppose by contradiction that (8.12) does not hold for all positive real numbers, then we can find $a, a^{\prime}>0$ with $a<a^{\prime}<a+\frac{1}{100}$ such that (8.12) holds for $a$ but not for $a^{\prime}$.

By Lemma 2.20 we have

$$
\left(\partial_{t}-\Delta\right) d_{t}\left(x_{0}(t), x\right) \geq-\frac{10}{3} r_{0}^{-1}
$$

whenever $d_{t}\left(x_{0}(t), x\right)>r_{0}$. Choose $\varphi: \mathbb{R} \rightarrow \mathbb{R}$ to be a smooth non-negative nonincreasing function such that $\varphi=1$ on $\left(-\infty, \frac{7}{8}\right], \varphi=0$ on $[1, \infty)$ and $\frac{2\left|\varphi^{\prime}\right|^{2}}{\varphi}+\left|\varphi^{\prime \prime}\right| \leq C_{0}$. 
Let $u: \mathcal{M} \rightarrow \mathbb{R}$ be defined by

$$
u(x)=\varphi\left(\frac{d_{t}\left(x_{0}(t), x\right)+\frac{10}{3} r_{0}^{-1} t}{A r_{0}}\right)\left(R+a^{\prime}(\mu+\nu)\right),
$$

and $u_{\min }(t)=\min \left\{\inf _{\mathcal{M}_{t}} u(\cdot), 0\right\}$.

By the same reasoning as Lemma 8.1 we can show the following inequality for all $t \in\left(0, t_{0}\right):$

$$
u_{\min }(t) \leq \lim \inf _{s \searrow t} u_{\min }(t)
$$

Let $T$ be the supremum of all $t$ such that $u(s) \geq-\frac{2 C_{0}}{\left(A r_{0}\right)^{2}}$ for all $s \in[0, t]$, where $C_{0}$ will be specified later. Then $T>0$ and by (8.15) we get

$$
u_{\min }(T) \leq-\frac{2 C_{0}}{\left(A r_{0}\right)^{2}}
$$

Since $u_{\min }(T)<0$, the minimum of $u$ is obtained at some point $x_{T} \in B_{T}\left(x_{0}(T), A r_{0}\right)$. Let $\mathbb{V}_{1}, \mathbb{V}_{2}, \mathbb{V}_{3}$ be the orthonormal eigenvectors of $\mathrm{Rm}$ corresponding to eigenvalues $\lambda \geq \mu \geq \nu$ at the tangent space of $x_{T}$. We extend them smoothly to a neighborhood $\mathcal{P}$ around $x_{T}$ in the following way: first extend them to a neighborhood of $x_{T}$ in $\mathcal{M}_{T}$ by parallel translation along radial geodesic emanating from $x_{T}$ using $\nabla^{g(T)}$, and then extend them in time to make them constant in time in the sense that $\nabla_{t} \mathbb{V}_{i}=0$, $i=1,2,3$, where $\nabla_{t}$ is the natural space-time extension of $\nabla^{g(t)}$ such that it is compatible with the metric, i.e. $\partial_{t}\langle X, X\rangle_{g(t)}=2\left\langle\nabla_{t} X, X\right\rangle_{g(t)}$. Then $\mathbb{V}_{1}, \mathbb{V}_{2}, \mathbb{V}_{3}$ is an orthonormal basis on $\mathcal{P}$, and $\Delta \mathbb{V}_{i}=0$ at $x_{T}, i=1,2,3$.

Let $\tilde{u}(x)=\left[\operatorname{Rm}\left(\mathbb{V}_{1}, \mathbb{V}_{1}\right)+\operatorname{Rm}\left(\mathbb{V}_{2}, \mathbb{V}_{2}\right)+\operatorname{Rm}\left(\mathbb{V}_{3}, \mathbb{V}_{3}\right)+a^{\prime}\left(\operatorname{Rm}\left(\mathbb{V}_{2}, \mathbb{V}_{2}\right)+\operatorname{Rm}\left(\mathbb{V}_{3}, \mathbb{V}_{3}\right)\right)\right]$. $\varphi\left(\frac{d_{t}\left(x_{0}(t), x\right)+\frac{10}{3} r_{0}^{-1} t}{A r_{0}}\right)$ for all $x \in \mathcal{P}$. Then it is easy to see that $\tilde{u}(x) \geq u(x)$ in $\mathcal{P}$, and the equality is achieved at $x_{T}$.

We can compute that

$$
\begin{aligned}
\left(\partial_{t}-\Delta\right) \tilde{u} & =-2 \nabla \varphi \nabla\left(\frac{\tilde{u}}{\varphi}\right)+\varphi \cdot\left(\partial_{t}-\Delta\right)\left[\operatorname{Rm}\left(\mathbb{V}_{1}, \mathbb{V}_{1}\right)+\left(a^{\prime}+1\right)\left(\operatorname{Rm}\left(\mathbb{V}_{2}, \mathbb{V}_{2}\right)+\operatorname{Rm}\left(\mathbb{V}_{3}, \mathbb{V}_{3}\right)\right)\right] \\
& +\left[\operatorname{Rm}\left(\mathbb{V}_{1}, \mathbb{V}_{1}\right)+\left(a^{\prime}+1\right)\left(\operatorname{Rm}\left(\mathbb{V}_{2}, \mathbb{V}_{2}\right)+\operatorname{Rm}\left(\mathbb{V}_{3}, \mathbb{V}_{3}\right)\right)\right] \cdot\left(\partial_{t}-\Delta\right) \varphi \\
& =-2 \nabla \varphi \nabla\left(\frac{\tilde{u}}{\varphi}\right)+\varphi \cdot \mathcal{I}+\mathcal{J} \cdot\left(\partial_{t}-\Delta\right) \varphi
\end{aligned}
$$

We estimate each term in (8.17) at $x_{T}$. First, recall that Rm evolves by $\left(\nabla_{t}-\right.$ $\Delta) \mathrm{Rm}=\mathrm{Rm}^{2}+\mathrm{Rm}^{\#}$ under Ricci flow, see [MT09, Proposition 3.19], where $(8.18)$

$$
M^{2}+M^{\#}=\left[\begin{array}{ccc}
\lambda^{2}+\mu \nu & 0 & 0 \\
0 & \mu^{2}+\lambda \nu & 0 \\
0 & 0 & \nu^{2}+\lambda \mu
\end{array}\right], \quad \text { for any matrix } \quad M=\left[\begin{array}{ccc}
\lambda & 0 & 0 \\
0 & \mu & 0 \\
0 & 0 & \nu
\end{array}\right]
$$


So by $\nabla \mathbb{V}_{i}=\Delta \mathbb{V}_{i}=\partial_{t} \mathbb{V}_{i}=0$ at $x_{T}$ we get

$$
\left(\partial_{t}-\Delta\right)\left(\operatorname{Rm}\left(\mathbb{V}_{i}, \mathbb{V}_{i}\right)\right)=\left(\left(\nabla_{t}-\Delta\right) \operatorname{Rm}\right)\left(\mathbb{V}_{i}, \mathbb{V}_{i}\right)=\left(\mathrm{Rm}^{2}+\mathrm{Rm}^{\#}\right)\left(\mathbb{V}_{i}, \mathbb{V}_{i}\right)
$$

at $x_{T}, i=1,2,3$, and hence

$$
\begin{aligned}
\mathcal{I}\left(x_{T}\right) & =\left(\lambda^{2}+\mu \nu\right)+\left(a^{\prime}+1\right)\left(\mu^{2}+\lambda \nu+\nu^{2}+\lambda \mu\right) \\
& \geq \lambda[\lambda+(a+1)(\mu+\nu)]+\left(a^{\prime}+1\right)\left(\mu^{2}+\nu^{2}\right)+\left(a^{\prime}-a\right) \lambda(\mu+\nu) \\
& \geq\left(a^{\prime}+1\right)\left(\mu^{2}+\nu^{2}\right)+\left(a^{\prime}-a\right) \lambda(\mu+\nu),
\end{aligned}
$$

where we used $\lambda+(a+1)(\mu+\nu) \geq 0$. Since $u\left(x_{T}\right)<0$, we have $\lambda<\left(a^{\prime}+1\right)|\mu+\nu|$ at $x_{T}$ and hence

$$
\left|\left(a^{\prime}-a\right) \lambda(\mu+\nu)\right| \leq\left(a^{\prime}-a\right)\left(a^{\prime}+1\right)(\mu+\nu)^{2} \leq \frac{a^{\prime}+1}{100}(\mu+\nu)^{2} \leq \frac{a^{\prime}+1}{50}\left(\mu^{2}+\nu^{2}\right) .
$$

Substituting this into (8.20) and using $\lambda<\left(a^{\prime}+1\right)|\mu+\nu|$ at $x_{T}$ again we get

$$
\begin{aligned}
\mathcal{I}\left(x_{T}\right) & \geq \frac{49}{50}\left(a^{\prime}+1\right)\left(\mu^{2}+\nu^{2}\right) \geq \frac{49}{100}\left(a^{\prime}+1\right)(\mu+\nu)^{2} \\
& \geq \frac{49}{200\left(a^{\prime}+1\right)}\left\{\left[\left(a^{\prime}+1\right)(\mu+\nu)\right]^{2}+\lambda^{2}\right\} \\
& \geq \frac{49}{400\left(a^{\prime}+1\right)}\left[\left(a^{\prime}+1\right)(\mu+\nu)+\lambda\right]^{2} \\
& =\frac{49}{400\left(a^{\prime}+1\right) \varphi^{2}} u_{\min }^{2}(T) .
\end{aligned}
$$

Then we estimate $\mathcal{J} \cdot\left(\partial_{t}-\Delta\right) \varphi$ at $x_{T}$ by using (8.13), $\varphi<0$ and $u_{\min }(T)<0$ as below

$$
\begin{aligned}
\left(\mathcal{J} \cdot\left(\partial_{t}-\Delta\right) \varphi\right)\left(x_{T}\right) & =\left[\lambda+\left(a^{\prime}+1\right)(\mu+\nu)\right]\left(\partial_{t}-\Delta\right) \varphi \\
& =\frac{1}{A r_{0}}\left[\varphi^{\prime}\left(\partial_{t}-\Delta\right) d_{t}\left(x_{0}(t), x\right)+\frac{10}{3} r_{0}^{-1}-\varphi^{\prime \prime} \frac{1}{A r_{0}}\right] u_{\min }(T) \\
& \geq \frac{1}{\left(A r_{0}\right)^{2} \varphi}\left|\varphi^{\prime \prime}\right| u_{\min }(T) .
\end{aligned}
$$

Next, since $\tilde{u}$ obtains its minimum on $\mathcal{P}$ at $x_{T}$ and $\tilde{u}\left(x_{T}\right)=u\left(x_{T}\right)=u_{\min }(T)$, we get

$$
\left(-2 \nabla \varphi \nabla\left(\frac{\tilde{u}}{\varphi}\right)\right)\left(x_{T}\right)=2 \frac{|\nabla \varphi|^{2}}{\varphi^{2}} u_{\min }(T)=2 \frac{\left|\varphi^{\prime}\right|^{2}}{\varphi^{2}} \frac{1}{\left(A r_{0}\right)^{2}} u_{\min }(T) .
$$


Now applying the maximum principle at $x_{T}$ and using (8.22), (8.23) and (8.24), we get

$$
\begin{aligned}
0 \geq\left(\partial_{t}-\Delta\right) \tilde{u}\left(x_{T}\right) & \geq \frac{49}{400\left(a^{\prime}+1\right) \varphi} u_{\min }^{2}(T)+\frac{1}{\left(A r_{0}\right)^{2} \varphi}\left|\varphi^{\prime \prime}\right| u_{\min }(T)+2 \frac{\left|\varphi^{\prime}\right|^{2}}{\left(A r_{0}\right)^{2} \varphi^{2}} u_{\min }(T) \\
& \geq \frac{49}{400\left(a^{\prime}+1\right) \varphi}\left[u_{\min }^{2}(T)+\frac{1}{\left(A r_{0}\right)^{2}}\left(\frac{2\left|\varphi^{\prime}\right|^{2}}{\varphi}+\left|\varphi^{\prime \prime}\right|\right) u_{\min }(T)\right] \\
& \geq \frac{49}{400\left(a^{\prime}+1\right) \varphi}\left[u_{\min }^{2}(T)+\frac{C_{0}}{\left(A r_{0}\right)^{2}} u_{\min }(T)\right],
\end{aligned}
$$

where we have used $\frac{2\left|\varphi^{\prime}\right|^{2}}{\varphi}+\left|\varphi^{\prime \prime}\right| \leq C_{0}$. Since $u_{\min }(T)<0$, (8.25) implies immediately $u_{\min }(T) \geq-\frac{C_{0}}{\left(A r_{0}\right)^{2}}$, which contradicts with (8.16). So $u_{\min }(t) \geq-\frac{2 C_{0}}{\left(A r_{0}\right)^{2}}$ for all $t \in$ $\left[0, t_{0}\right)$. Letting $A \rightarrow \infty$ we get $R+a^{\prime}(\mu+\nu) \geq 0$ on $\mathcal{M}$, which contradicts the assumption of $a^{\prime}$. So (8.12) holds for all $a>0$, and hence by the argument at beginning the conclusion of the Lemma follows.

The next lemma says that in a 3 -dimensional manifold with Ric $\geq 0$, no singularity can form within finite distance along a minimizing geodesic covered by final time-slices of strong $\delta$-necks. We prove it by a contradiction argument, suppose the assertion does not hold, then by the condition of Ric $\geq 0$, we can show that the blow-up limit of the 'singularity' is a smooth cone, and there is a Ricci flow whose final time-slice is in the smooth part of the cone, which is impossible.

Lemma 8.3. For any sufficiently small $\delta>0$ the following holds: Let $(M, g)$ be a 3 dimensional Riemannian manifold with Ric $\geq 0$. Let $\gamma:\left[0, s_{0}\right) \rightarrow M$ (where $s_{0} \in \mathbb{R}_{+} \cup\{\infty\}$ ) be a unit speed minimizing geodesic such that $R(\gamma(s))$ does not stay bounded for $s \rightarrow s_{0}$, and assume there are constants $c, \varphi>0$ such that all points on $\gamma$ are centers of strong $\delta$-necks on the time interval $[-c, 0]$, and the strong $\delta$-necks have $\varphi$-positive curvature.

Then $s_{0}=\infty$.

Proof. Suppose by contradiction that $s_{0}<\infty$. Let $\eta$ be from Lemma 2.15.

Since every point on $\gamma$ is the center of some strong $\delta$-neck, we get that $\gamma$ lies inside some open subset $N \subset M$ that is diffeomorphic to $S^{2} \times(0,1)$ and which is covered by final time-slices of strong $\delta$-necks. Consider the length metric induced by the Riemannian metric on $N$, and then $N^{\prime}$ be the completion of $N$. Then $N^{\prime}$ is a disjoint union of $N$ and a single point $p$.

Consider the rescalings $i N^{\prime}$ for all $i \in \mathbb{N}$. Then by the Bishop-Gromov volume comparison we can deduce that for any $d>0$, the $d$-balls $B^{i N^{\prime}}(p, d)$ in $i N^{\prime}$ are uniformly totally bounded. Therefore, by Gromov's compactness theorem, we have 
the following Gromov-Hausdorff convergence by passing to a subsequence $\left\{i_{k} N^{\prime}\right\}$ :

$$
\left(i_{k} N^{\prime}, p\right) \stackrel{k \rightarrow \infty}{\longrightarrow}\left(X, p_{\infty}\right)
$$

We shall show that $X$ is a smooth metric cone with cone point $p_{\infty}$, and the convergence is actually smooth on $X_{0}=X-\left\{p_{\infty}\right\}$.

Let $x \in B\left(p, s_{0}\right)-\{p\} \subset N^{\prime}$, then by Lemma 2.15 we get

$$
R^{-1 / 2}(x) \leq \eta d(p, x) \quad \text { on } \quad B\left(p, s_{0}\right)-\{p\} .
$$

We claim that there exists $C>0$ such that

$$
R^{-1 / 2}(x) \geq C^{-1} d(p, x) \quad \text { on } \quad B\left(p, s_{0}\right)-\{p\} .
$$

Suppose not, then there exists a sequence $\left\{x_{k}\right\} \subset B\left(p, s_{0}\right)-\{p\}$ such that

$$
R^{-1 / 2}\left(x_{k}\right) \leq C_{k}^{-1} d\left(p, x_{k}\right),
$$

where $C_{k} \rightarrow \infty$ as $k \rightarrow \infty$. We abbreviate $d\left(p, x_{k}\right)$ as $d_{k}$ and $R\left(x_{k}\right)$ as $R_{k}$.

Since $x_{k}$ is the center of a $\delta$-neck, there is a diffeomorphism onto its image $\phi_{k}$ : $\left(-\delta^{-1}, \delta^{-1}\right) \times S^{2} \rightarrow N^{\prime}$ under which $\left(N^{\prime}, x_{k}\right)$ is $\delta$-close to $\left(-\delta^{-1}, \delta^{-1}\right) \times S^{2}$ at scale $R_{k}^{-1 / 2}$. Let $U_{k}=\phi_{k}\left((-100,100) \times S^{2}\right)$, then $U_{k}$ separates $N^{\prime}$ into two components.

Suppose $x \in B\left(p, s_{0}\right)-\{p\}$ is not in $U_{k}$, then it is easy to see either

$$
d(x, p)>d_{k}+10 R_{k}^{-1 / 2}, \quad \text { or } \quad d(x, p)<d_{k}-10 R_{k}^{-1 / 2} .
$$

In other words, we have

$$
B\left(p, d_{k}+10 R_{k}^{-1 / 2}\right)-B\left(p, d_{k}-10 R_{k}^{-1 / 2}\right) \subset U_{k} .
$$

Applying the Bishop-Gromov volume comparison on $N^{\prime}$, we have $r^{-2} \operatorname{vol}(\partial B(p, r))$ is non-increasing for all $r \in\left(0, s_{0}\right)$. In particular, let $v_{0}=s_{0}^{-2} \operatorname{vol}\left(\partial B\left(p, s_{0}\right)\right)$, then $r^{-2} \operatorname{vol}(\partial B(p, r)) \geq v_{0}$ for all $0<r<s_{0}$. So by (8.31) we can estimate the volume of $U_{k}$ from below:

$$
\begin{aligned}
\operatorname{vol}\left(U_{k}\right) & \geq \int_{d_{k}-10 R_{k}^{-1 / 2}}^{d_{k}+10 R_{k}^{-1 / 2}} \operatorname{vol}(\partial B(p, r)) d r \geq \int_{d_{k}-10 R_{k}^{-1 / 2}}^{d_{k}+10 R_{k}^{-1 / 2}} v_{0} r^{2} d r \\
& \geq \frac{9}{16} \int_{d_{k}-10 R_{k}^{-1 / 2}}^{d_{k}+10 R_{k}^{-1 / 2}} v_{0} d_{k}^{2} d r=\frac{45}{4} v_{0} d_{k}^{2} R_{k}^{-1 / 2},
\end{aligned}
$$

where in the third inequality we used (8.29), which implies $d_{k}-R_{k}^{-1 / 2} \geq \frac{3}{4} d_{k}$ for large $k$.

By the closeness of the metric on $U_{k}$ with the standard cylindrical metric at scale $R_{k}^{-1 / 2}$, we get an upper bound on the volume of $U_{k}$ :

$$
\operatorname{vol}\left(U_{k}\right) \leq 2 \cdot R_{k}^{-3 / 2} \cdot 200 \cdot 8 \pi=3200 \pi R_{k}^{-3 / 2}<3200 \pi C_{k}^{-2} d_{k}^{2} R_{k}^{-1 / 2},
$$


where we used (8.29) in the last inequality. Combining (8.32) with (8.33) we get $C_{k}^{2} \leq \frac{12800 \pi}{45 v_{0}}$, which is impossible for large $k$. Thus there exists $C>0$ such that (8.28) holds.

Therefore, by (8.28) we see that the convergence on $X_{0}$ is smooth. So there is $v_{1}>0$ such that $d^{-2} \operatorname{vol}\left(\partial B^{X}\left(p_{\infty}, d\right)\right)=v_{1}$ for all $d \in(0, \infty)$. So by the rigidity of volume comparison, we see that any Jacobi field along any geodesic emanating from $p_{\infty} \in X$ has linear growth, which implies that $X$ is a smooth metric cone. By (8.27), $X_{0}$ is nowhere flat.

Since all points in a neighborhood of $p$ are centers of strong $\delta$-necks on $[-c, 0]$, which has $\varphi$-positive curvature. So under the blow-up rescalings this implies that any point $x \in X_{0}$ is the center of a strong $2 \delta$-neck on $\left[-\frac{1}{2} c, 0\right]$, which has non-negative sectional curvature. This contradicts the fact that open pieces in non-flat cones cannot arise as the result of Ricci flow with non-negative curvature [MT09, Prop 4.22].

Theorem 8.4. (Theorem 1.3) Let $(M, g)$ be a 3d complete Riemannian manifold with Ric $\geq 0$. There exist $T>0$ and a smooth Ricci flow $(M, g(t))$ with $g(0)=g$ defined on $[0, T)$. Moreover, if $T<\infty$, then $\lim \sup _{t \nearrow T}|\operatorname{Rm}|(x, t)=\infty$ for all $x \in M$.

Proof. First we assume $M$ is orientable. By Theorem 7.14 there is a generalized singular Ricci flow $(\mathcal{M}, g(t))$ starting from $(M, g)$, and by Lemma 8.2, $\mathcal{M}$ has nonnegative Ricci curvature.

Let $x_{0} \in M$. Suppose $x_{0}$ survives until $t_{0}>0$ in $\mathcal{M}$. We claim that the component of $\mathcal{M}_{t}$ that contains $x_{0}(t)$ is complete for all $t \in\left(0, t_{0}\right]$. Suppose not, then $\sup _{B_{t}\left(x_{0}(t), A\right)} R=\infty$ for some $A>0$ and $t \in\left(0, t_{0}\right]$. By Lemma 3.3 and 3.4, we can find a minimizing geodesic $\gamma:[0,1) \rightarrow \mathcal{M}_{t}$ such that $\lim _{s \rightarrow 1} R(\gamma(s))=\infty$, and there exist $c, \varphi>0$ such that for all $s$ close to $1, \gamma(s)$ are centers of strong $\delta$-necks on $[-c, 0]$, which have $\varphi$-positive curvature. This contradicts Lemma 8.3.

Since Ric $\geq 0$, for any $A>0$, the parabolic neighborhood $P\left(x_{0}, A, t_{0}\right)$ is contained in $\bigcup_{t \in\left[0, t_{0}\right]} B_{t}\left(x_{0}(t), A\right)$, which is relatively compact. So every point in $M$ survives until $t_{0}$. Let $T \in(0, \infty]$ be the supremum of all times until which $x_{0}$ survives. Then $T$ is also the supremum of the survival times of points in $M$. Suppose $T<\infty$, since $\mathcal{M}$ is forward 0-complete, we have $\lim _{\sup _{t} \nearrow_{T}}|\operatorname{Rm}|(x(t))=\infty$ for all $x \in M$. So the spacetime restricted on the subset $\bigcup_{t \in[0, T)} M(t)$ is the desired smooth Ricci flow.

Now suppose $M$ is not orientable. Let $\widehat{M} \rightarrow M$ be the 2-fold orientation covering. By Theorem 7.14 , there are a generalized singular Ricci flow $(\widehat{\mathcal{M}}, g(t))$ starting from $\widehat{M}$, and an isometry $\sigma: \widehat{\mathcal{M}} \rightarrow \widehat{\mathcal{M}}$ that acts free on the subset of points that can survive back to $\widehat{M}$. As before, there exists $T \in(0, \infty]$ such that $\widehat{M}$ survives on $[0, T)$, and $\limsup _{t \nearrow T}|\operatorname{Rm}|(x(t))=\infty$ for all $x \in \widehat{M}$ if $T<\infty$. The smooth Ricci flow claimed in the theorem is the quotient of $\bigcup_{t \in[0, T)} \widehat{M}(t)$ by the free action of $\sigma$. 


\section{REFERENCES}

[BBM11] Laurent Bessières, Gérard Besson, and Sylvain Maillot. Ricci flow on open 3-manifolds and positive scalar curvature. Geometry \& Topology, 15(2):927-975, 2011.

[BCRW19] Richard H. Bamler, Esther Cabezas-Rivas, and Burkhard Wilking. The Ricci flow under almost non-negative curvature conditions. Inventiones, 2019.

[BK17a] Richard H. Bamler and Bruce Kleiner. Ricci flow and diffeomorphism groups of 3manifolds. arXiv:1712.06197, 2017.

[BK17b] Richard H. Bamler and Bruce Kleiner. Uniqueness and stability of Ricci flow through singularities. http://arxiv.org/abs/1709.04122, 2017.

[Bre18] S. Brendle. Ancient solutions to the Ricci flow in dimension 3. http://arxiv.org/abs/1811.02559, 2018.

$\left[\mathrm{CCG}^{+} 10\right]$ By Bennett Chow, Sun-chin Chu, David Glickenstein, Christine Guenther, James Isenberg, Tom Ivey, Dan Knopf, Peng Lu, Feng Luo, and Lei Ni. The Ricci flow: techniques and applications. Part III: geometric-analytic aspects. American Mathematical Society, $163,2010$.

[Che09] Binglong Chen. Strong uniqueness of the Ricci flow. J. Differential Geom., 82(2), 2009.

[CK88] Christopher B. Croke and Hermann Karcher. Volumes of small balls on open manifolds: lower bounds and examples. Transactions of the American Mathematical Society, 309(2):753-753, 1988.

[CRW15] Esther Cabezas-Rivas and Burkhard Wilking. How to produce a Ricci flow via CheegerGromoll exhaustion. Journal of the European Mathematical Society, 17(12):3153-3194, 2015.

[CXZ13] Bing Long Chen, Guoyi Xu, and Zhuhong Zhang. Local pinching estimates in 3-Dim ricci flow. Mathematical Research Letters, 20(5):845-855, 2013.

[Ham82] Richard S Hamilton. Three-manifolds with positive Ricci curvature. J. Differ. Geom., 17:255-306, 1982.

[Ham86] R. S. Hamilton. Four manifolds with positive curvature operator. J. Differ. Geom., 24:153-179, 1986.

[HN14] Hans-Joachim Hein and Aaron Naber. New logarithmic Sobolev inequalities and an $\epsilon$-regularity theorem for the Ricci flow. Communications on Pure and Applied Mathematics, 67(9):1543-1561, 2014.

[KL08] Bruce Kleiner and John Lott. Notes on Perelman's papers. Geom. Topol., 2, 2008.

[KL17] Bruce Kleiner and John Lott. Singular Ricci flows I. Acta Math., 219:65-134, 2017.

[KL18] Bruce Kleiner and John Lott. Singular Ricci flows II. https://arxiv.org/abs/1804.03265, 2018.

[Lai19] Yi Lai. Ricci flow under local almost non-negative curvature conditions. Advances in Mathematics, 343:353-392, 2019.

[MT09] John Morgan and Gang Tian. Ricci flow and the Poincare conjecture. arXiv:math/0607607, 2009.

[Ni06] Lei Ni. A note on Perelman's LYH inequality. Comm. Anal. Geom., 14:883-905, 2006.

[Per02] Grisha Perelman. The entropy formula for the Ricci flow and its geometric applications. http://arxiv.org/abs/math/0211159, 2002.

[Per03a] Grisha Perelman. Finite extinction time for the solutions to the Ricci flow on certain three-manifolds. http://arxiv.org/abs/math/0307245, 2003.

[Per03b] Grisha Perelman. Ricci flow with surgery on three-manifolds. http://arxiv.org/abs/math/0303109, 2003.

[Pet06] Peter Petersen. Riemannian geometry. Springer, second edition, 2006.

[Shi87] Wan-Xiong Shi. Deforming the metric on complete Riemannian manifolds. Journal of differential geometry, 30(1):223-301, 1987. 
[ST17] Miles Simon and Peter M. Topping. Local mollification of Riemannian metrics using Ricci flow, and Ricci limit spaces. http://arxiv.org/abs/1706.09490, pages 1-25, 2017.

[Top19] Peter Topping. Ricci flow and Ricci Limit Spaces. Https://Arxiv.Org/Abs/1904.11375, 2019 .

E-mail address: yilai@berkeley.math.edu

Department of Mathematics, University of California, Berkeley, CA 94720, USA 